\title{
Biosimilars for the Treatment of Chronic Inflammatory Diseases: A Systematic Review of Published Evidence
}

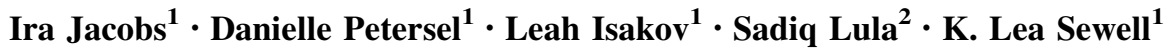

Published online: 25 November 2016

(c) The Author(s) 2016. This article is published with open access at Springerlink.com

\begin{abstract}
Background Clinicians are required to assimilate, critically evaluate, and extrapolate information to support appropriate use of biosimilars across indications.

Objectives The objective of this study was to systematically collate all published data in order to assess the weight (quantity and quality) of available evidence for each molecule and inform and support healthcare decisionmaking in chronic inflammatory diseases.

Methods MEDLINE ${ }^{\circledR}$, EMBASE ${ }^{\circledR}$, and ISI Web of Science ${ }^{\circledR}$ were searched to September 2015. Selected conference proceedings were searched from 2012 to July 2015. Studies disclosing biosimilars with unique identifiers were categorized by originator, study type, and indication. Risk of bias assessments were performed. Intended copies were differentiated as commercially available agents without evidence of rigorous comparative biosimilarity evaluations. Results Proposed biosimilars for adalimumab, etanercept, infliximab, and rituximab are reported in the published literature. Across indications, approved biosimilars infliximab CT-P13, SB2, and etanercept SB4 have published studies involving the largest number of patients or healthy subjects
\end{abstract}

Leah Isakov was an employee of Pfizer at the time the study was conducted.

Electronic supplementary material The online version of this article (doi:10.1007/s40259-016-0201-6) contains supplementary material, which is available to authorized users.

Ira Jacobs

ira.jacobs@pfizer.com

1 Pfizer Essential Health, Pfizer Inc., 235 East 42nd Street, New York, NY 10017-5755, USA

2 Envision Pharma Group, London, UK
( $n=1405,743$, and 734, respectively), mostly in rheumatoid arthritis. At data cut-off, only CT-P13 had published data in ankylosing spondylitis $(n=250$; randomized control trial) and ulcerative colitis/Crohn's disease $(n=336$; observational studies). Published data were not available for ongoing studies in psoriasis patients. Four intended copies were identified in published studies (total: $n=1430$; $n=1372$ in observational studies). Thematic analysis of non-empirical publications showed that indication extrapolation remains an issue, particularly for gastroenterologists. Conclusions While most agents display a moderate to high degree of similarity to their originator in the published studies identified, large discrepancies persist in the overall amount and type of data available in the public domain. Significant gaps exist particularly for intended copies, reinforcing the need to maintain a clear differentiation between these molecules and true biosimilars.

\section{Key Points}

There is a significant body of evidence in the published literature to support infliximab biosimilars CT-P13, SB2, and etanercept biosimilar SB4 for rheumatoid arthritis, but knowledge gaps still exist both in the amount and type of data available. These gaps are more pronounced for other molecules across chronic inflammatory diseases, and most pronounced for intended copies.

Rheumatoid arthritis is the first indication for which biosimilars have been launched and experience here will influence how the broader sector evolves.

Ongoing dissemination of data by all manufacturers is imperative to support adoption of biosimilars. 


\section{Introduction}

A biosimilar or follow-on biologic [1] is a biologic medicinal product that contains a version of the active substance of an already authorized original biologic medicinal product [2-4]. Over 700 biosimilar products are reported to be in preclinical and clinical trials [5]. For biosimilar drug approval, data must be generated to establish whether a biosimilar can safely and effectively be used instead of the originator product.

Although highly efficacious, biologic therapy for chronic inflammatory disease is expensive [6]. The expectation among patients, treating physicians, and healthcare providers is that biosimilars should be highly similar in efficacy, comparable in safety, including immunogenicity, but lower in price than their reference products [7]. The introduction of biosimilars can help expand access to safe and effective treatment options for clinicians and patients [6].

Several biosimilars are now available for the treatment of chronic inflammatory diseases. CT-P13 (Remsima ${ }^{\circledR} / \mathrm{In}$ flectra $^{\circledR}$, an infliximab biosimilar) was the first EU-approved monoclonal antibody $(\mathrm{mAb})$ biosimilar, obtaining market authorization in September 2013 for all approved indications of the innovator drug, including rheumatoid arthritis (RA), ankylosing spondylitis (AS), psoriatic arthritis (PsA), psoriasis, Crohn's disease (CD), and ulcerative colitis (UC). In 2014, Health Canada initially approved CT-P13 for all approved indications of infliximab except for UC and CD [8]; Health Canada subsequently approved CT-P13 for additional indications, including CD, fistulizing $\mathrm{CD}$, and $\mathrm{UC}$ [9].The addition of $\mathrm{CD}$, fistulizing $\mathrm{CD}$, and $\mathrm{UC}$ to the approved indications was granted on the basis of similarity between CT-P13 and the reference product Remicade ${ }^{\circledR}$ in product quality, mechanism of action, disease pathophysiology, safety profile, dosage regimen, and on clinical experience with the reference product [9]. In February 2016, the US Food and Drug Administration (FDA) Arthritis Advisory Committee approved CT-P13 for all indications of the reference product, making it the first biosimilar mAb therapy to be reviewed by the FDA for licensure in the USA.

In November 2015, the European Medicines Agency (EMA) recommended marketing approval for SB4 (Benepali $^{\circledR}$, an etanercept biosimilar) for the treatment of RA, AS, PsA, and psoriasis [7]. On 1 June 2016, the EMA Committee for Medicinal Products for Human Use announced a positive opinion on SB2 (Flixabi ${ }^{\circledR}$, an infliximab biosimilar) for the treatment of RA, CD, UC, AS, PsA, and psoriasis [10]. On 12 July 2016, the FDA advisory panel voted in favor of recommending approval for ABP 501, Amgen's proposed biosimilar of adalimumab, to treat seven chronic inflammatory diseases, including RA,
PsA, CD, UC, and plaque psoriasis [11]. Finally, on 13 July 2016, the FDA Arthritis Advisory Committee unanimously voted to recommend approval of Sandoz's GP2015 (an etanercept biosimilar) as the totality of the evidence demonstrated that GP2015 is similar to US-licensed Enbrel ${ }^{\circledR}$ (etanercept) [12].

Similarity to the reference product in terms of quality characteristics, biologic activity, efficacy, and safety must be established before biosimilar products can be marketed in the USA, Europe, or other regulated countries [2]. Numerous batches of the innovator reference product are routinely tested to establish the characteristic range. Manufacturers must provide high-quality evidence of similar efficacy and safety outcomes, including a comprehensive immunogenicity assessment to satisfy the stringent requirements for EMA and FDA approval.

In contrast, 'intended copies' are copies of originator biologics that have not undergone rigorous comparative evaluations as recommended by the leading regulatory authorities, but are nevertheless being sold in some countries. There is a lack of information about the efficacy and safety of intended copies compared with the originator. Furthermore, they may have clinically noticeable differences in quality characteristics, pharmacokinetics, efficacy, or safety. The extent and quality of available data between proposed biosimilars and intended copies often greatly differ [13].

A comprehensive systematic literature review (SLR) was recently undertaken to identify, collate, and summarize all published empirical evidence on named biosimilars and intended copies of originator $\mathrm{mAbs}$ and fusion proteins (Jacobs et al. [14]; see page 489 of this issue). The original analysis aimed to summarize the range of available data and number and diversity of publications describing biosimilars (for the treatment of chronic inflammatory disease along with other disease areas, including oncology). Here, we explore in greater depth the findings for biosimilars indicated for chronic inflammatory disease.

\section{Methods}

\subsection{Systematic Literature Review}

A detailed description of the methods used in this SLR can be found in Jacobs et al. [14] (see page 489 of this issue). First, the search strategy captured $\mathrm{mAb}$ and fusion protein terms; then included the different terminologies for biosimilar products, such as biosimilars, subsequent entry biologics, follow-on biologics, follow-on proteins, biocomparables, biogenerics, similar biotherapeutic products, or intended copies and biobetters (which were analyzed separately). Publications were required to contain both 
"mAb/fusion protein" and "biosimilar" terms. Here we report the results of biosimilars indicated for the treatment of RA (active, moderate, severe), psoriasis or plaque psoriasis, PsA, AS, CD, UC, scleroderma, and dermatomyositis. The search results were filtered using the study designs of interest and controlled vocabulary and free-text terms were applied.

MEDLINE $^{\circledR} / \mathrm{MEDLINE}^{\circledR}$ In-Process and EMBASE $^{\circledR}$ (searched using the OVIDSP interface), and ISI Web of Science $^{\circledR}$ were searched from database inception to 3 September 2015. The search was carried out on 27 April 2015 and repeated on 3 September 2015 to capture the latest full-text publications. In addition to the publication search and in order to identify recent studies not yet published as full-text articles and/or to provide additional data from previously published studies, a hand search of relevant conference proceedings (17 conferences) was conducted between 1 January 2012 and 31 July 2015 (Electronic Supplementary Material [ESM] Table S1).

The search result was limited to references published in the English language. For this analysis, biosimilars are differentiated from intended copies based on meeting the rigorous regulatory requirements for biosimilarity, as described by major regulatory health authorities such as the EMA, FDA, the World Health Organization (WHO), Health Canada, the Pharmaceuticals Medical Devices Agency/Japan Ministry for Health Labour and Welfare (PMDA/MHLW), or the Korean Ministry of Food and Drug Safety (MFDS) in the Republic of Korea [15, 16]. Guidelines for biosimilar approval have also been issued by Administración Nacional de Medicamentos, Alimentos y Tecnología Médica (ANMAT) in Argentina, Agência Nacional de Vigilância Sanitária (ANVISA) in Brazil, and Comisión Federal para la Protección contra Riesgos Sanitario (COFEPRIS) in Mexico. However, it should be noted that products approved in Latin American countries might not have been authorized following as strict a regulatory process as is required for approval of biosimilars in the USA or EU [17]. The biosimilar approval pathway was recently updated by India's Central Drugs Standard Control Organization (CDSCO) [18]. Other markets have issued guidance on biosimilars, although evaluation of the biosimilar approval pathways by regulatory authorities outside of the major markets was considered beyond the scope of this review.

Results from the SLR were split further into two components: the empirical analysis, which focused on peerreviewed publications of clinical, pharmacovigilance, and observational empirical data, and the non-empirical analysis, which included opinion pieces or commentaries, publications describing product-related patient support programs, and articles on manufacturing and supply issues. Non-empirical articles were further classified into general thematic categories to summarize key topics being discussed in the field of biosimilar medicines. Empirical studies were also categorized by type: preclinical (including analytical, functional, or nonclinical studies), clinical (pharmacokinetic/safety trials and preliminary or comparative safety/efficacy trials), or observational (prospective, retrospective, and post-marketing) studies. Results of clinical trial searches (from ClinicalTrials.gov) were also included as a separate subsection, and planned or ongoing clinical trials of biosimilars, where results were yet to be published, were explored.

\subsection{Risk of Bias (Quality) Assessment}

In accordance with the Preferred Reporting Items for Systematic Reviews and Meta-Analyses (PRISMA) guidelines, a validated tool matched to the study type was used to assess the strength/validity of the empirical data for each individual study $[19,20]$. In cases where multiple publications were retrieved for the same study, quality assessments were only conducted on the first original publication or first full-text publication. Further information on each tool is provided in the ESM.

The assessment of the quality of randomized controlled trials (RCTs) was carried out using recommendations from the National Institute for Health and Care Excellence (NICE) single technology appraisal (STA) manufacturer's template [21] and the Jadad scoring system [22] (ESM Table S2, ESM Fig. S1).

The Downs and Black [23] instrument was used to assess the quality of all non-randomized studies. As studies published as abstracts in conference proceedings have limited information, the Downs and Black instrument was modified to include only the most relevant qualifying parameters ( $n=12$ of 26) for quality assessment, as listed in ESM Tables S3 and S4. Pharmacoeconomic studies were evaluated using Drummond's checklist for assessing economic evaluations [24]. Animal studies were assessed using SYRCLE's (SYstematic Review Centre for Laboratory animal Experimentation) risk of bias tool [25]. Conference abstracts of analytical/nonclinical and economic studies were not evaluated, as suitable tools were not available at the time of analysis. Full-text analytical and cell-based studies were also not evaluated for the same reason.

\section{Results}

\subsection{Literature and Conference Search}

The literature search returned 1,991 publications that were identified through title and abstract screening, and those 
relevant to the topic of biosimilars were retained (768 publications in total). Of the included references, 301 (39\%) addressed biosimilars for the treatment of chronic inflammatory conditions. The numbers of publications included in the analysis are presented in a PRISMA flow diagram (shown in ESM Fig. S2). Of note, in cases where encore (or duplicate) publications were retrieved for studies, the information was compared with the original (first published article) and excluded if no additional data were provided. If new data were identified, encore publications were included along with the original publication. This would be reflected in the overall publication count but not in the overall study count. Named biosimilars (i.e., where a unique identifier was provided) were identified in 55 studies (96 publications) in chronic inflammatory disease (Fig. 1).

\subsection{Preclinical and Pharmacokinetic/Safety Data in Healthy Subjects}

\subsubsection{Adalimumab Biosimilars}

Studies of adalimumab biosimilars are presented in Table 1.

ABP 501 (Amgen) Pharmacokinetic/safety studies for ABP 501 have been reported [28, 26, 27]. In healthy subjects, ABP 501 was reported to demonstrate pharmacokinetic equivalence to adalimumab and, overall, no safety or immunogenicity concerns were raised. The combined results of analytical and nonclinical in vitro studies suggest a high degree of similarity between ABP 501 and adalimumab, despite some variations reported in structural composition.

Exemptia $^{T M}$ (Cadila Healthcare) Bandyopadhyay and coworkers [40] reported on the physicochemical properties and in vivo pharmacokinetic and toxicology profile of Exemptia $^{\mathrm{TM}}$ in the journal Biosimilars and concluded that the molecule displayed highly similar characteristics to adalimumab. It should be noted that studies from non-indexed publications were not included in this review, and therefore the data from this publication were not extracted. Based on the information from this study and other information which came to the attention of the authors, it seems reasonable to assume that this molecule may be considered a biosimilar and not an intended copy; however, compliance with the EMA, FDA, and WHO requirements is not yet fully transparent.

GP2017 (Sandoz) A published nonclinical study found GP2017 to be well-tolerated among tested species (mouse, rabbit, and monkey), and that it displayed a similar pharmacokinetic profile to that of adalimumab [37, 36, 35].

PF-06410293 (Pfizer) Analytical/nonclinical studies reporting results of peptide mapping experiments comparing PF-06410293 and adalimumab revealed highly similar primary sequence structures [38].

SB5 (Samsung Bioepis) SB5 has been studied in pharmacokinetic/safety studies in healthy volunteers to establish bioequivalence with adalimumab [41]. The pharmacokinetic data were not reported, but all parameters were found to fall within the required range for equivalence. The majority of adverse events (AEs) were mild in severity and comparable to the extent observed in adalimumab-treated healthy subjects.

\subsubsection{Etanercept Biosimilars}

Studies of etanercept biosimilars are presented in Table 2. ENIA11 (TuNEX ${ }^{\circledR} ;$ Mycenax Biotech/TSH Biopharm Corp) One pharmacokinetic/safety RCT was identified for ENIA11, wherein 23 healthy male subjects were randomized to study medication and reported similar pharmacokinetic/pharmacodynamic outcomes, with no serious AEs compared with etanercept [43, 42].

HD2O3 (Davictrel ${ }^{T M}$; Hanwha Chemical) In a doubleblind RCT comparing HD203 with etanercept in healthy subjects, pharmacokinetic/pharmacodynamic outcomes were similar between the two drugs and no serious AEs were observed [44].

LBEC0101 (LG Life Sciences) One pharmacokinetic/ safety RCT for LBEC0101 in healthy volunteers was found. It reported similar pharmacokinetic/pharmacodynamic outcomes between this biosimilar and etanercept [47].

SB4 (Benepali ${ }^{\circledR}$; Samsung Bioepis) In a pharmacokinetic/safety trial, pharmacokinetic equivalence was reported and SB4 was well-tolerated in healthy male subjects with a similar safety profile to etanercept $[48,49]$.

AVG01 (Avent ${ }^{T M}$; Avesthagen) One analytical/nonclinical study reported that AVG01 was highly similar to etanercept [52].

GP2015 (Sandoz) Two nonclinical studies were identified for GP2015. Both reported that GP2015 was highly similar to etanercept with regard to target binding, antitumor necrosis factor (TNF)- $\alpha$ biologic activity, and pharmacokinetic exposure [51,53].

\subsubsection{Infliximab Biosimilars}

Studies of infliximab biosimilars are presented in Table 3. BOW015 (Infimab ${ }^{\circledR}$; Ranbaxy Laboratories) A recent announcement from Ranbaxy Laboratories indicates that BOW015 has been discontinued. Prior to this, the pharmacokinetic, safety, and immunogenicity profiles of BOW015 and infliximab were compared in healthy volunteers $[54,55]$. Studied pharmacokinetic parameters and safety outcomes were reported to be comparable between 


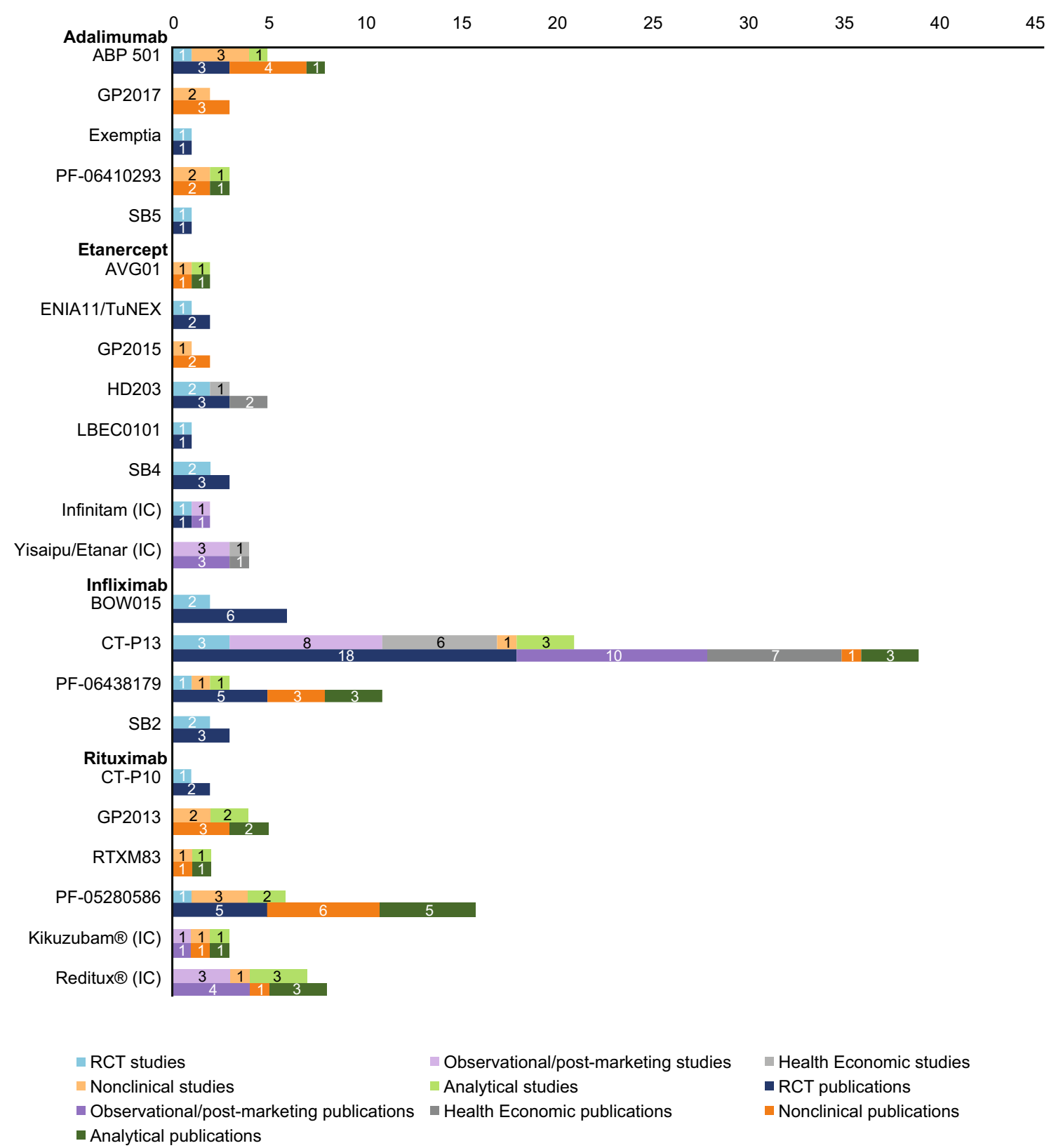

Fig. 1 Frequency of publications of reported named biosimilars in chronic inflammatory diseases. Note: publications were classified into the most relevant category, which in some cases was more than one. Therefore, the number of publications classified into each therapeutic area category does not sum to the total number of publications. For

the biosimilar and the originator. Within the SLR timeframe, no analytical or nonclinical data had been published or reported for BOW015 to validate its structural and functional biosimilarity to infliximab.

PF-06438179 (Pfizer) The pharmacokinetics and safety attributes of PF-06438179 have been evaluated in healthy volunteers $[94,95,93,98,99,97,96]$. Reports of studies confirmed pharmacokinetic bioequivalence of example, overlap in licensed indications for originators/biosimilars led to multiple categorization. Among the empirical references, several (seven) include both nonclinical and human data, and as such have been classified into both categories. IC Intended copy, $R C T$ randomized controlled trial

PF-06438179 as compared with infliximab, with similar incidences of AEs and lower occurrence of immunogenicity in PF-06438179 versus infliximab-treated subjects. The biochemical characteristics and in vitro/ in vivo biologic activity of PF-06438179 have been studied and reported. The data suggest that PF-06438179 exhibits high structural and functional similarity to infliximab. 


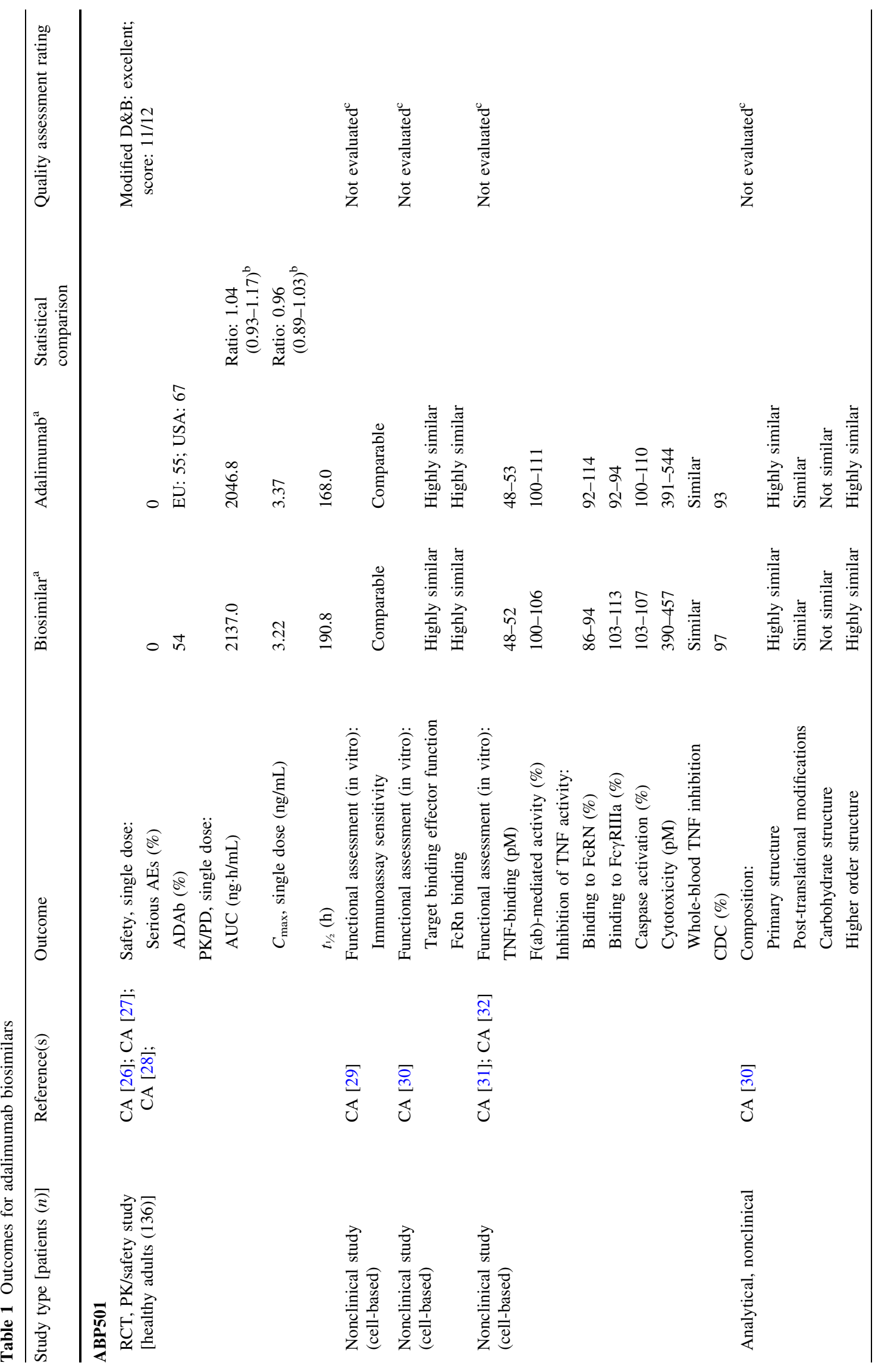




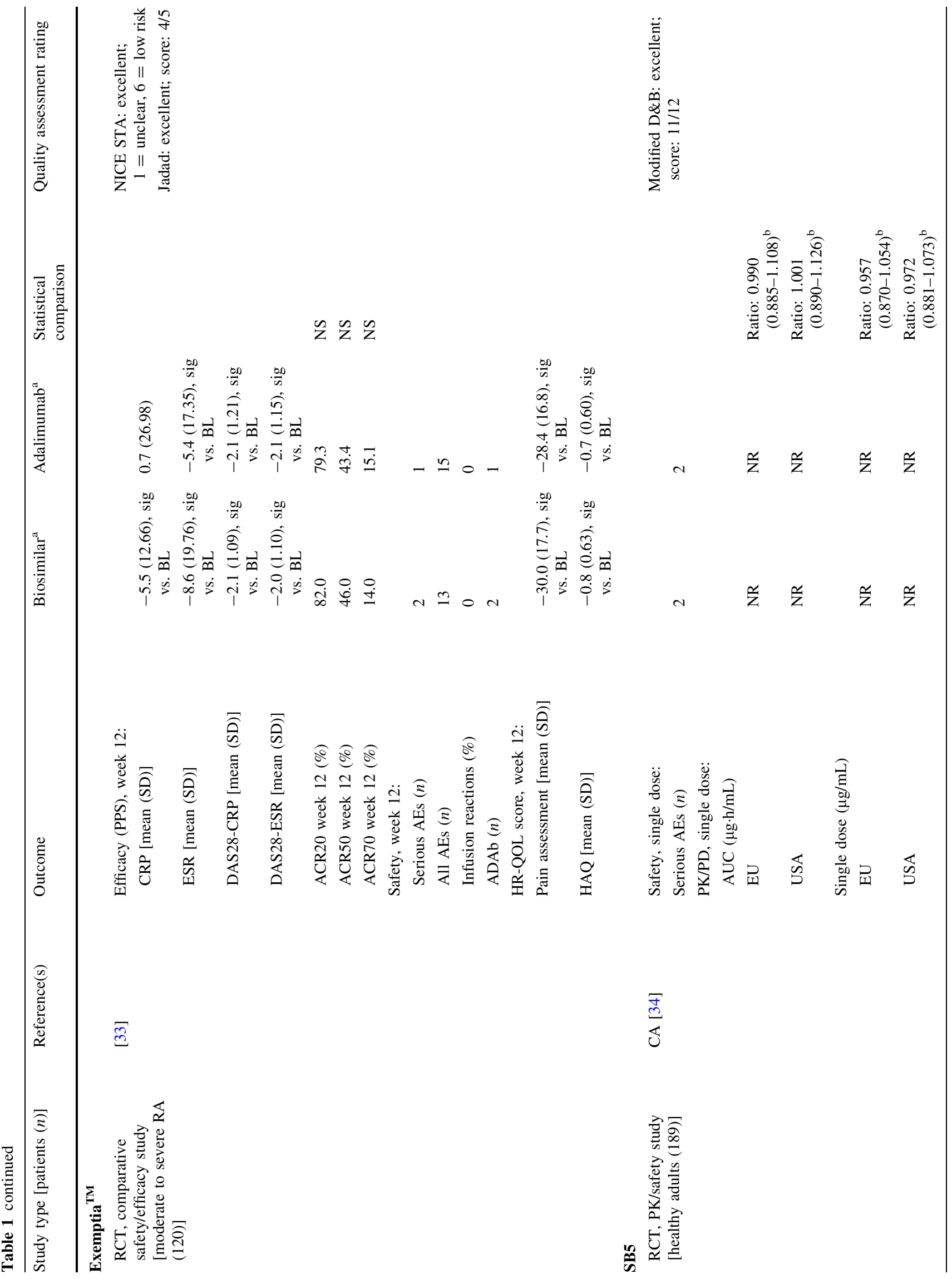




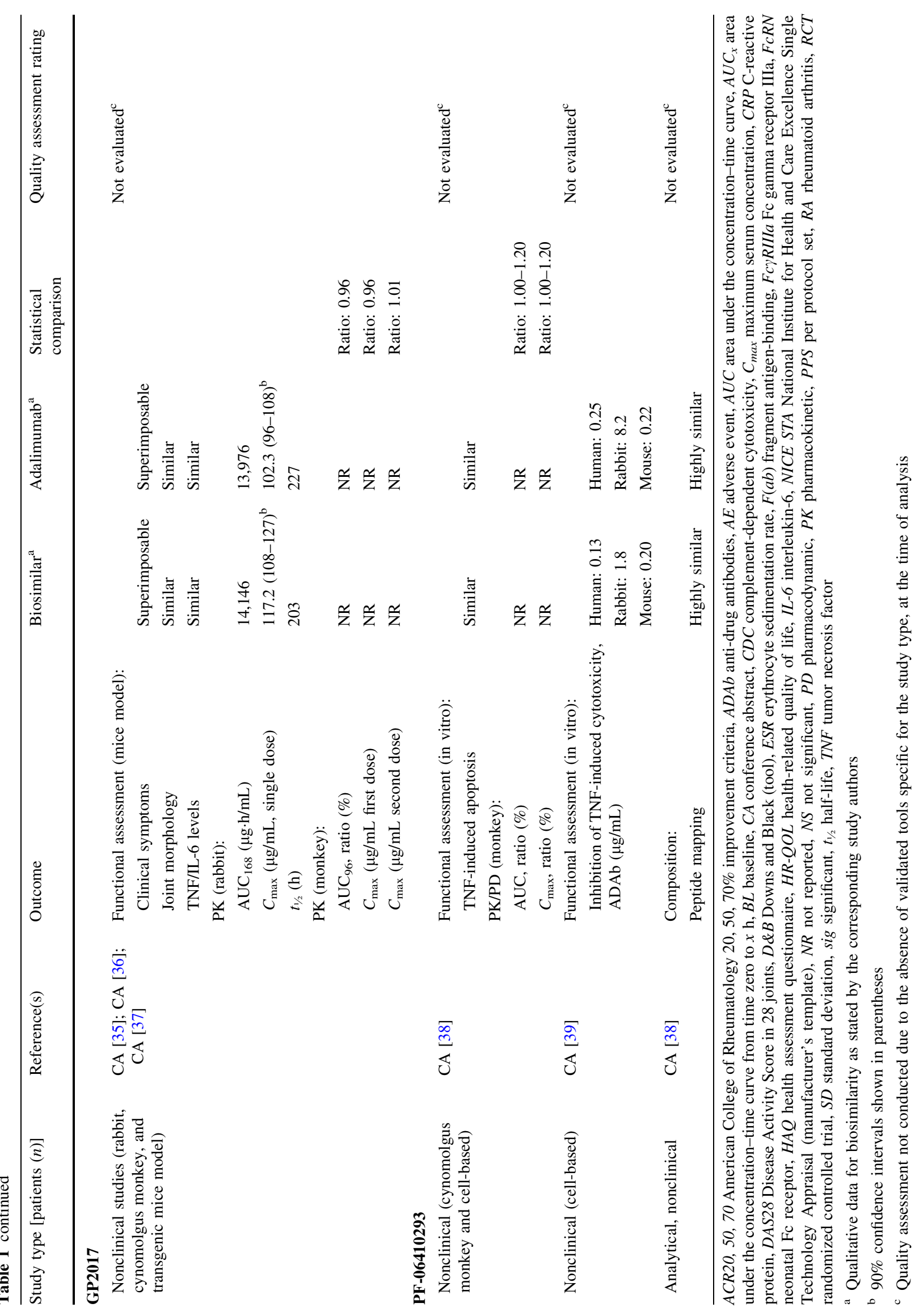




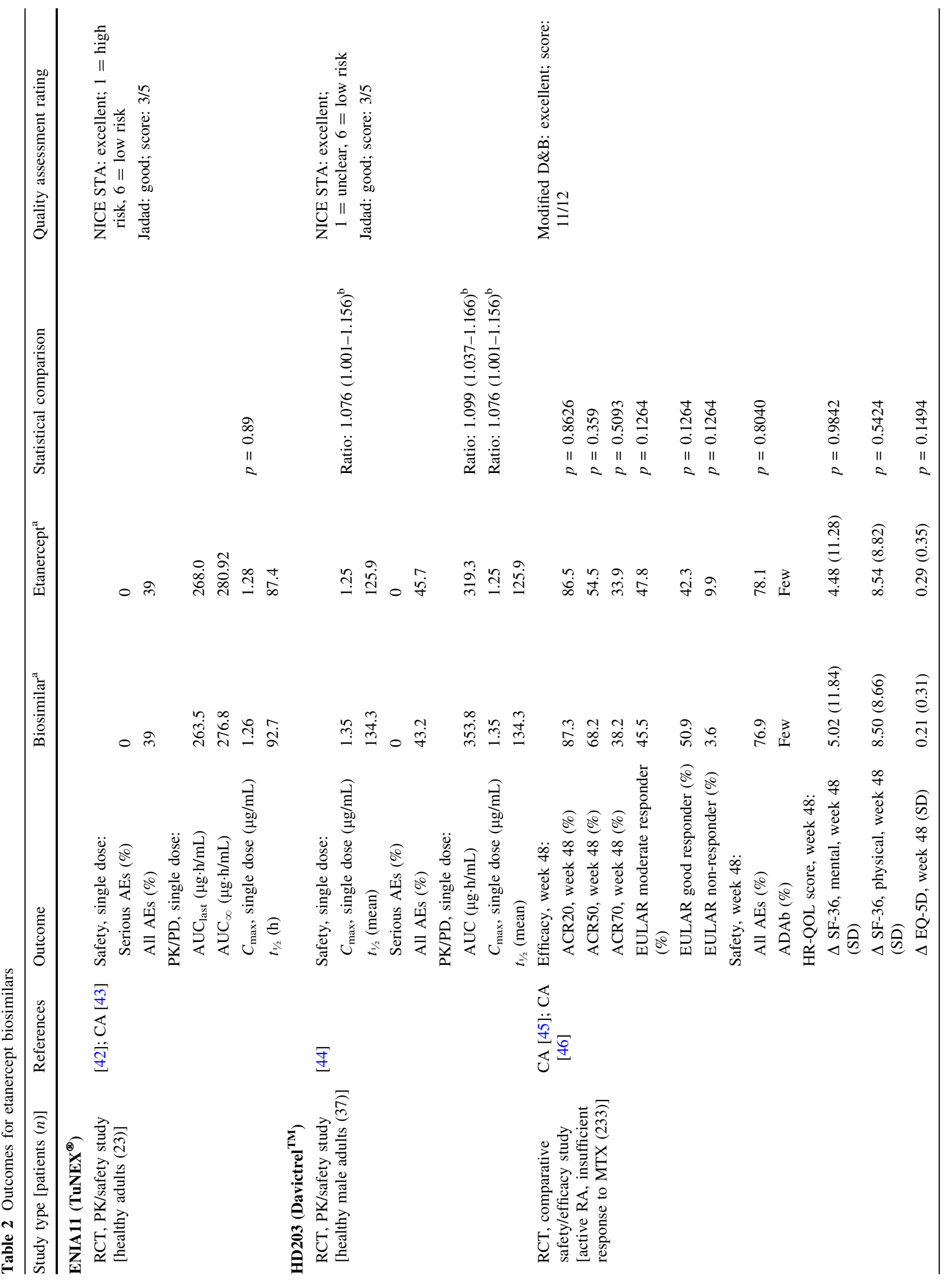




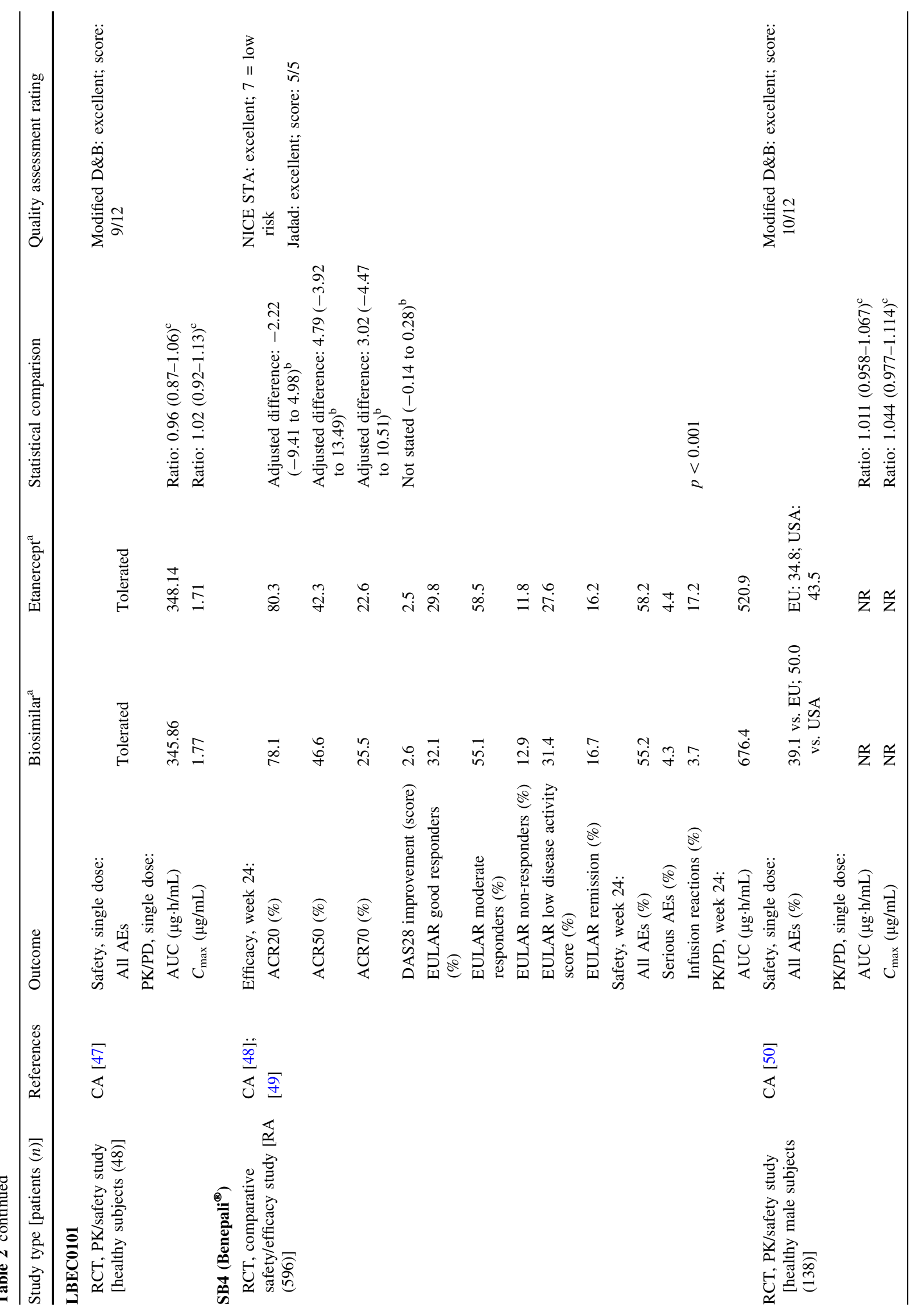




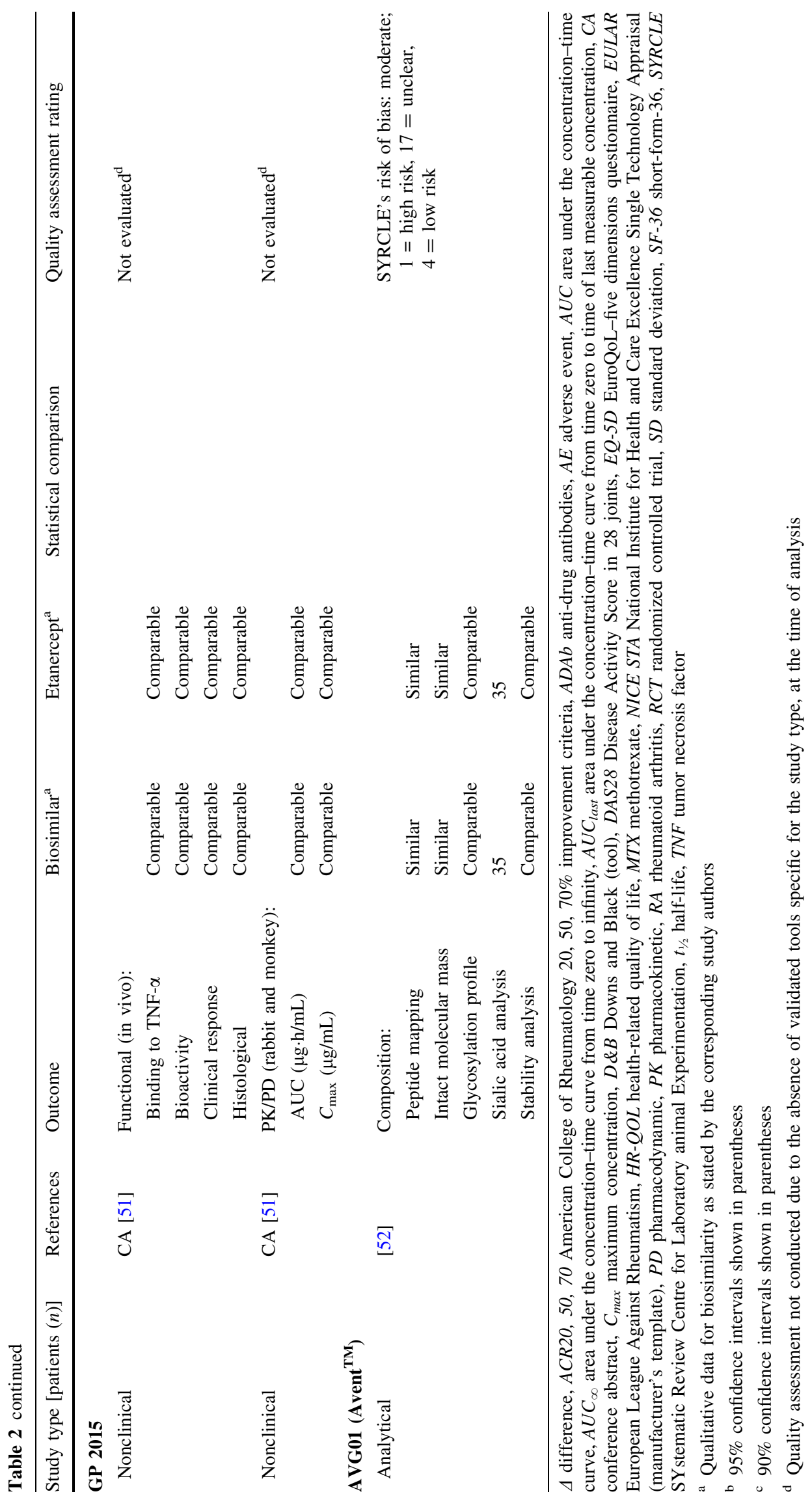




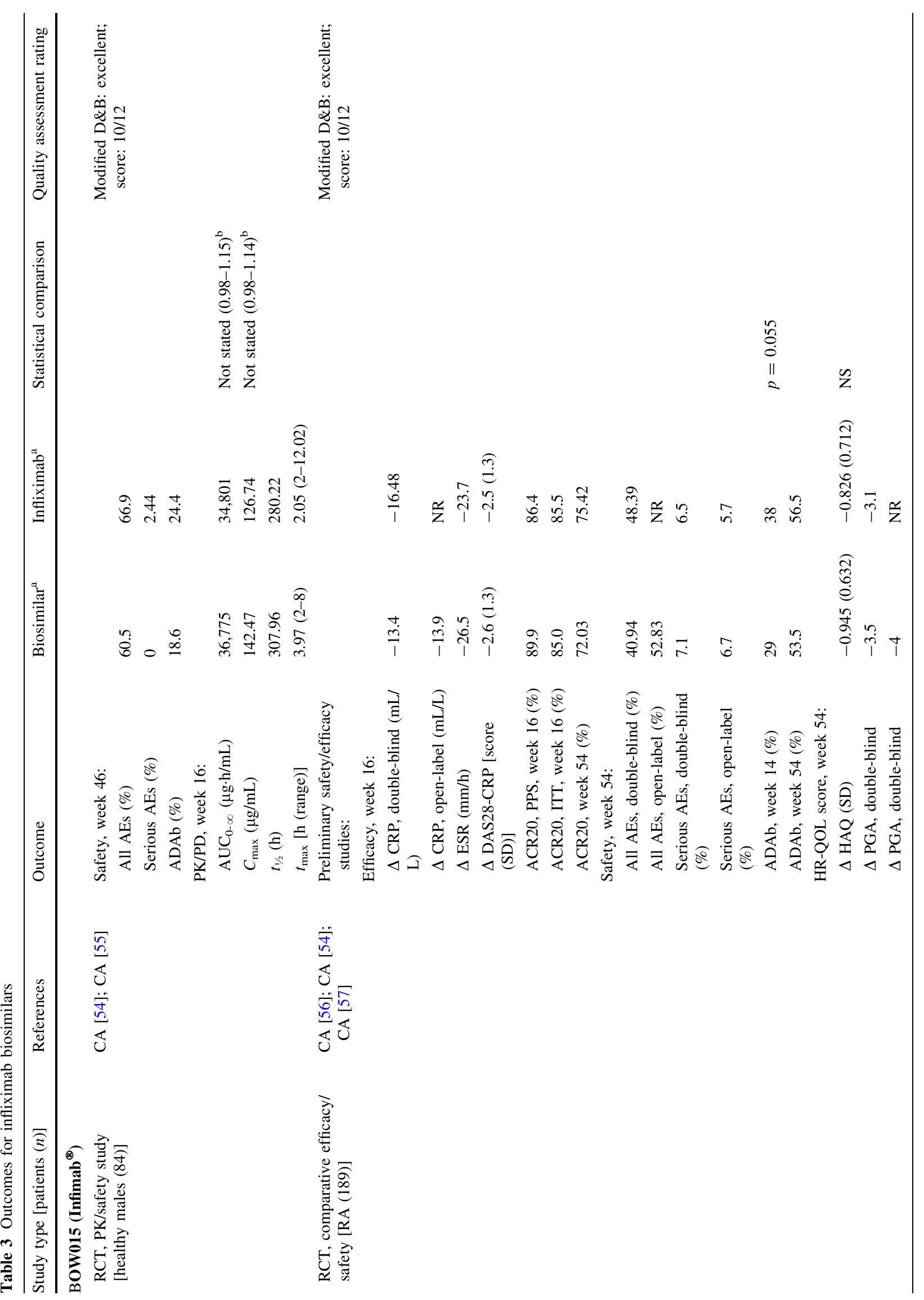




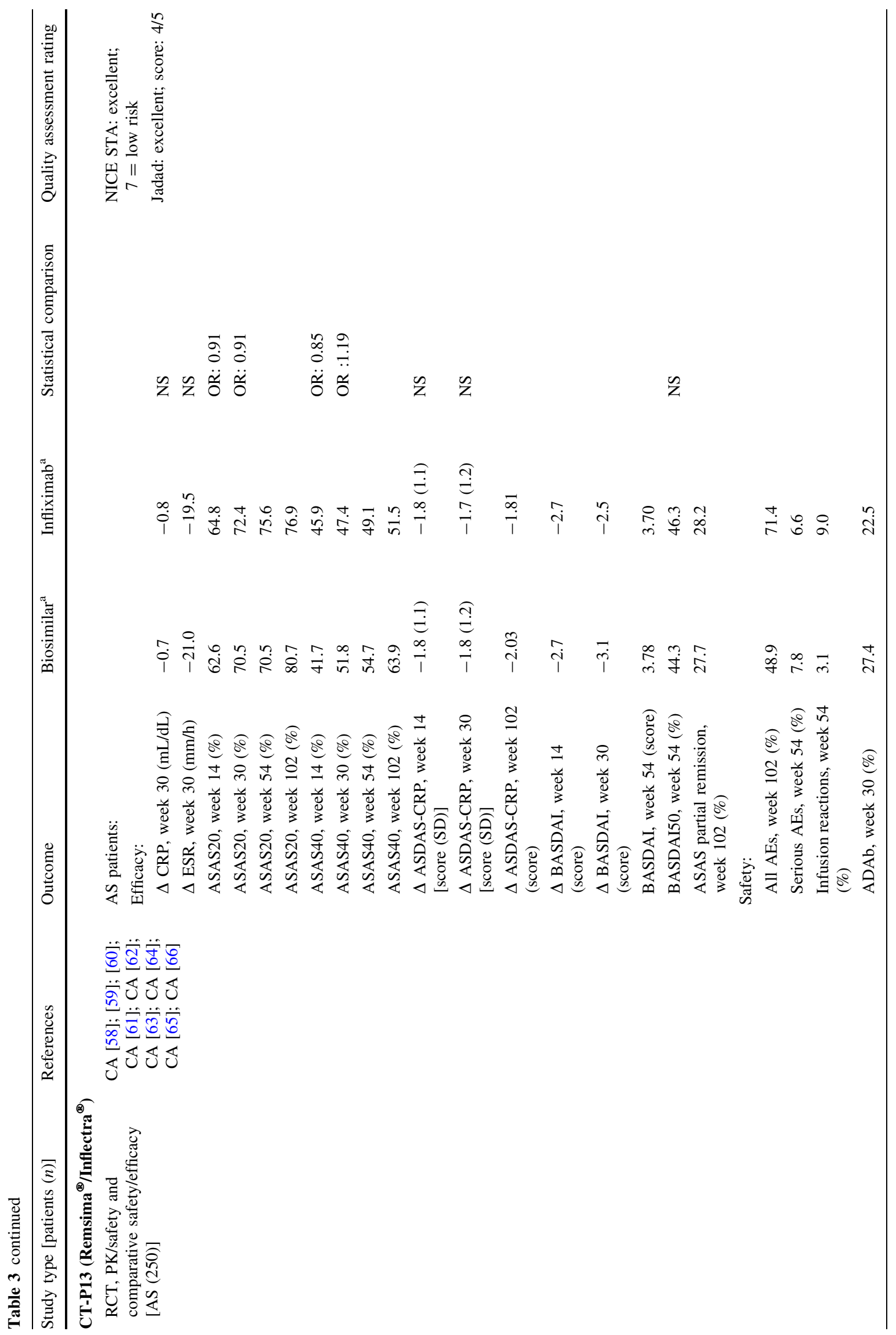




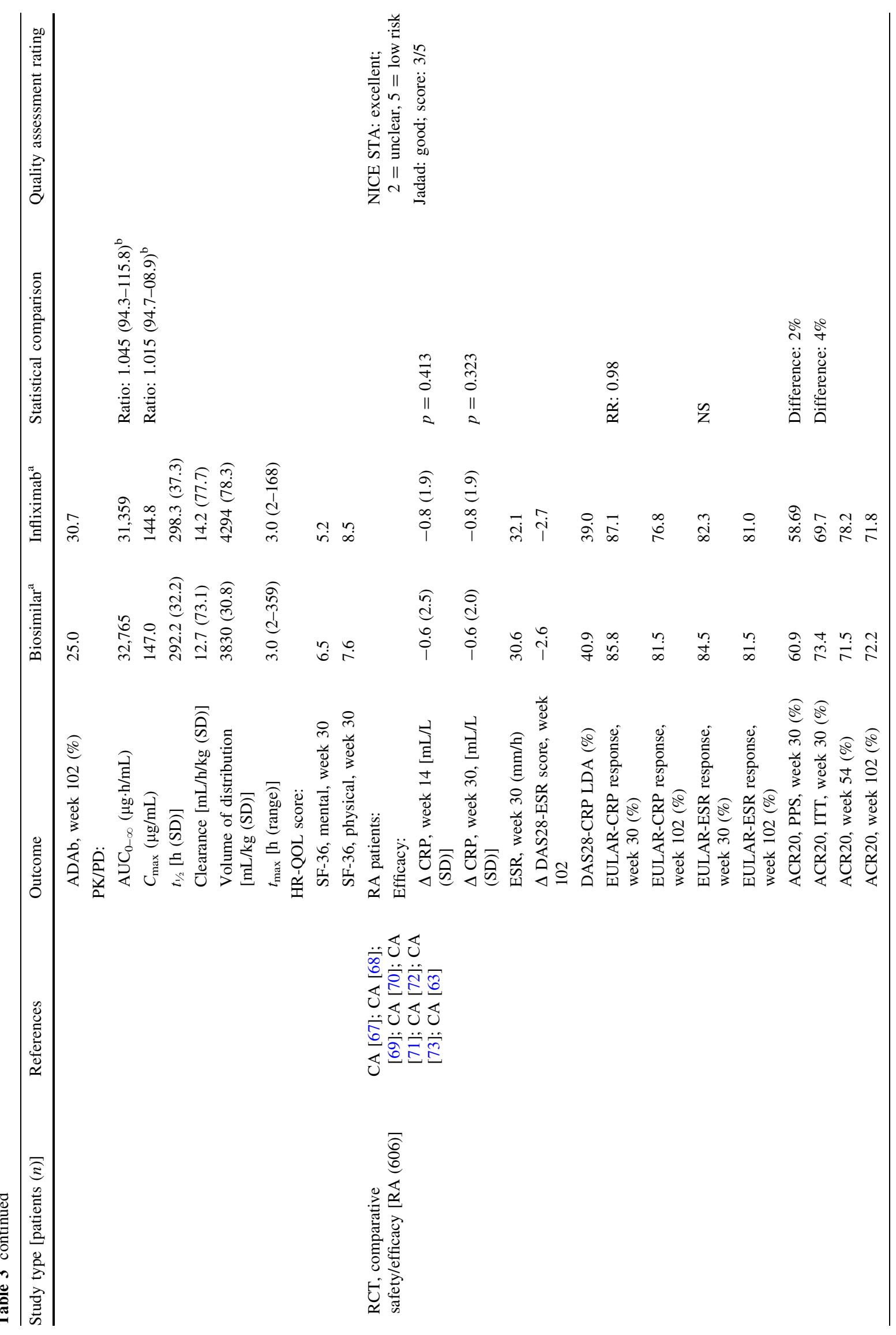




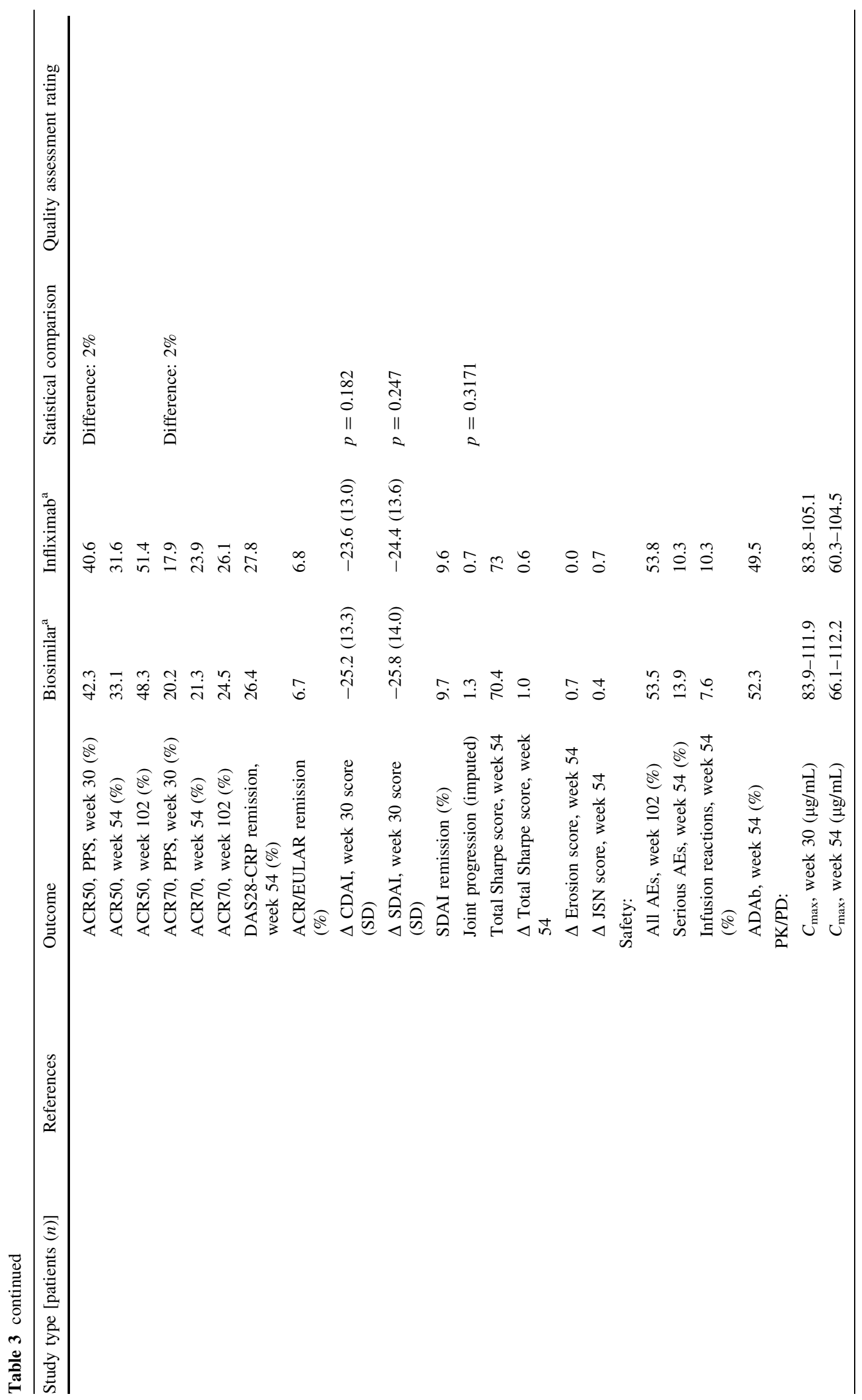




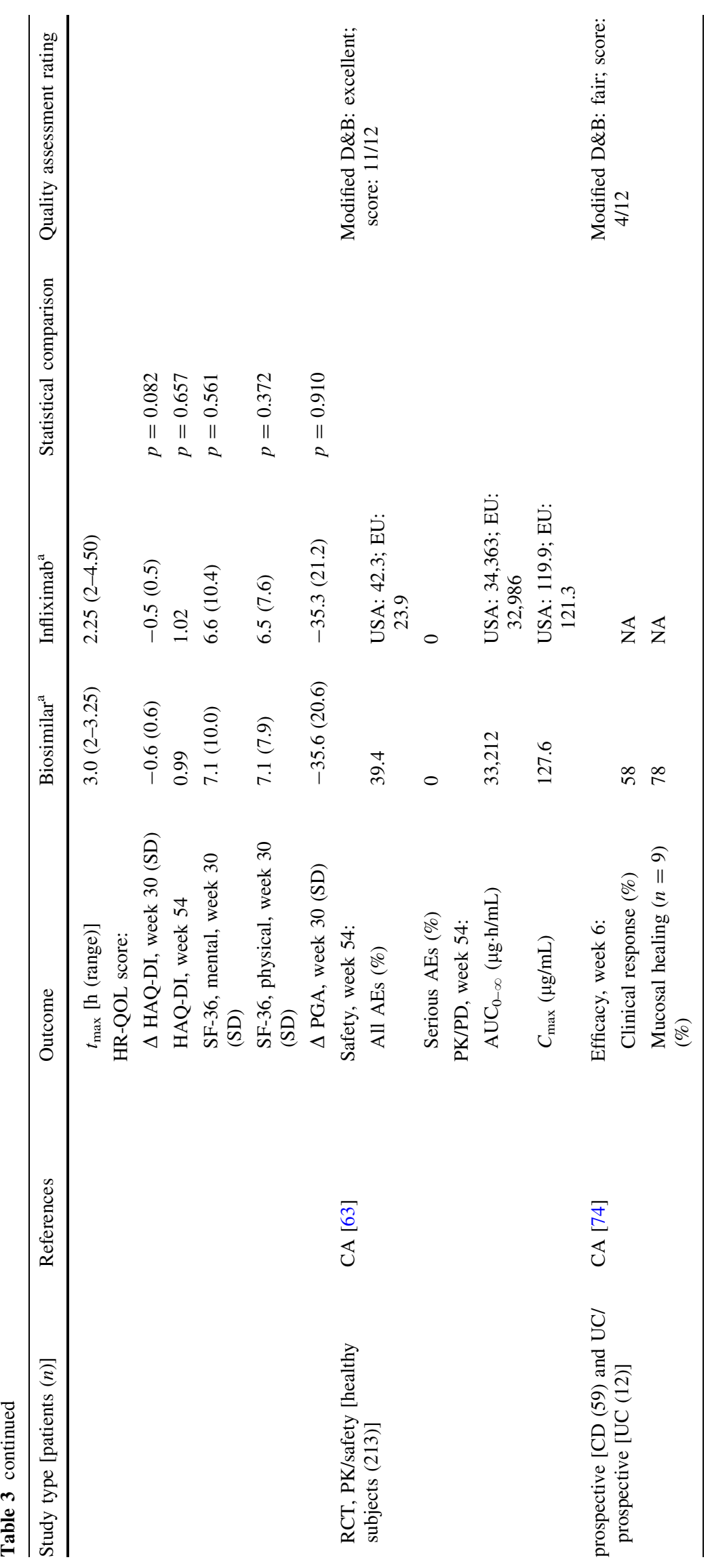




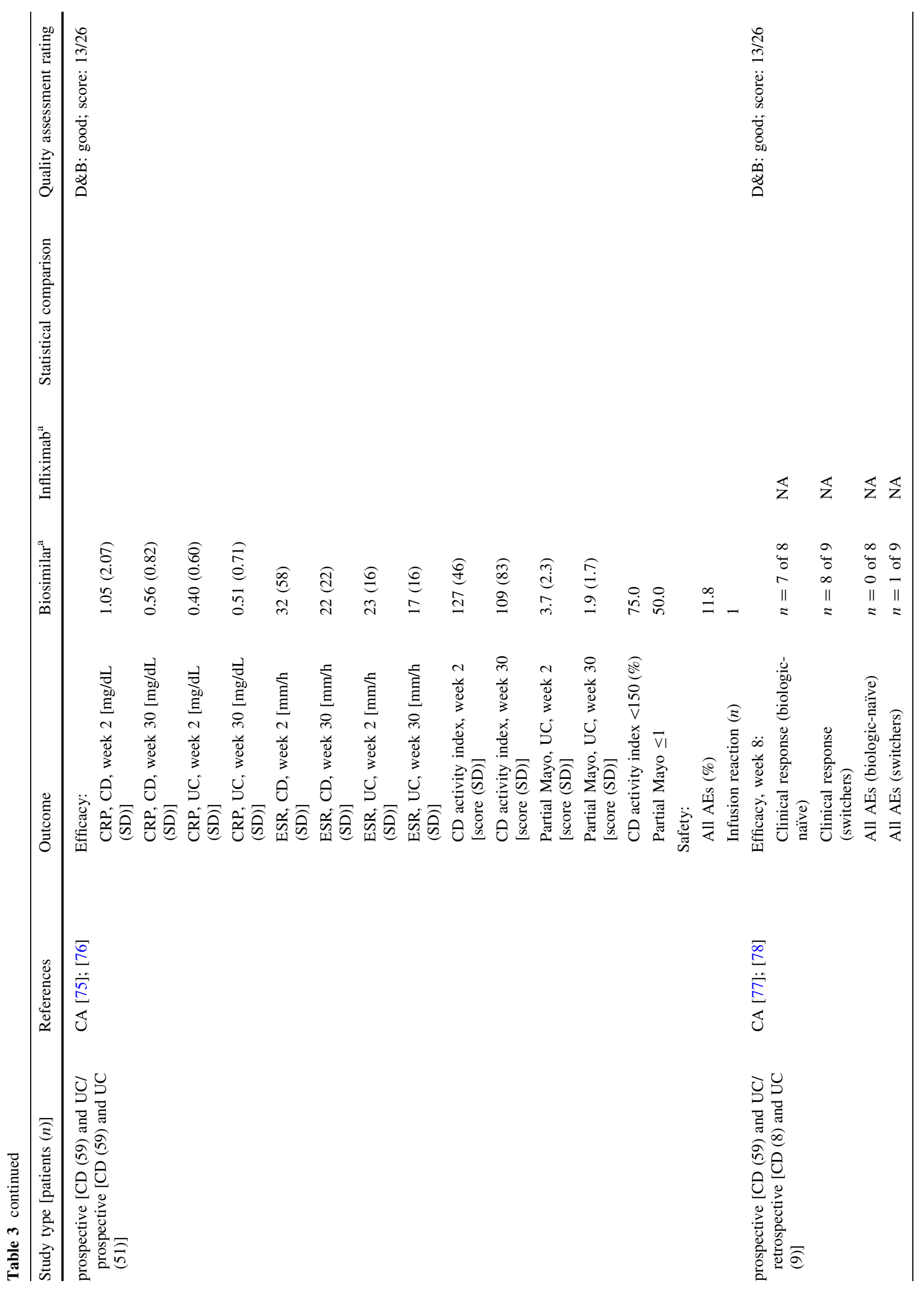




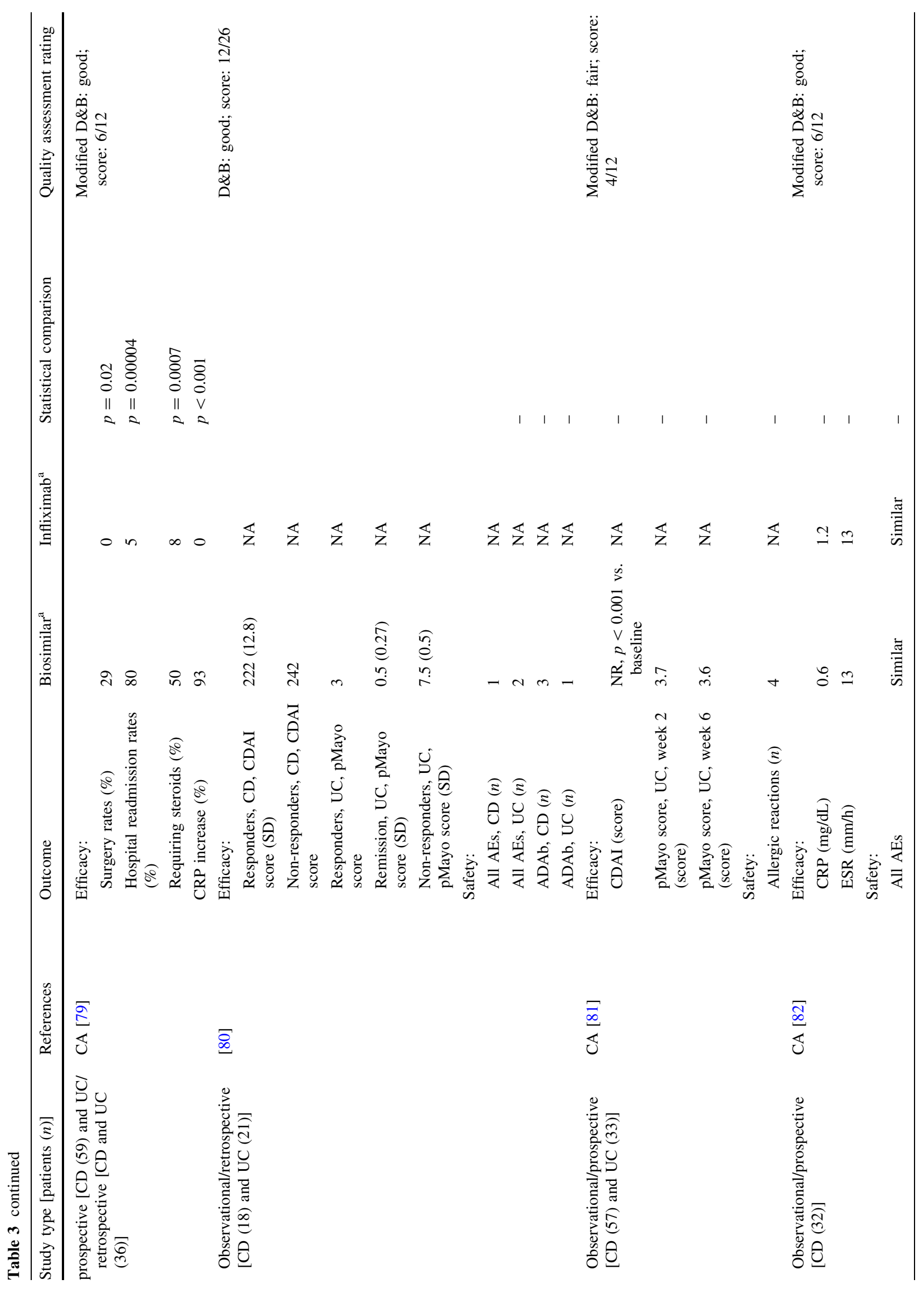




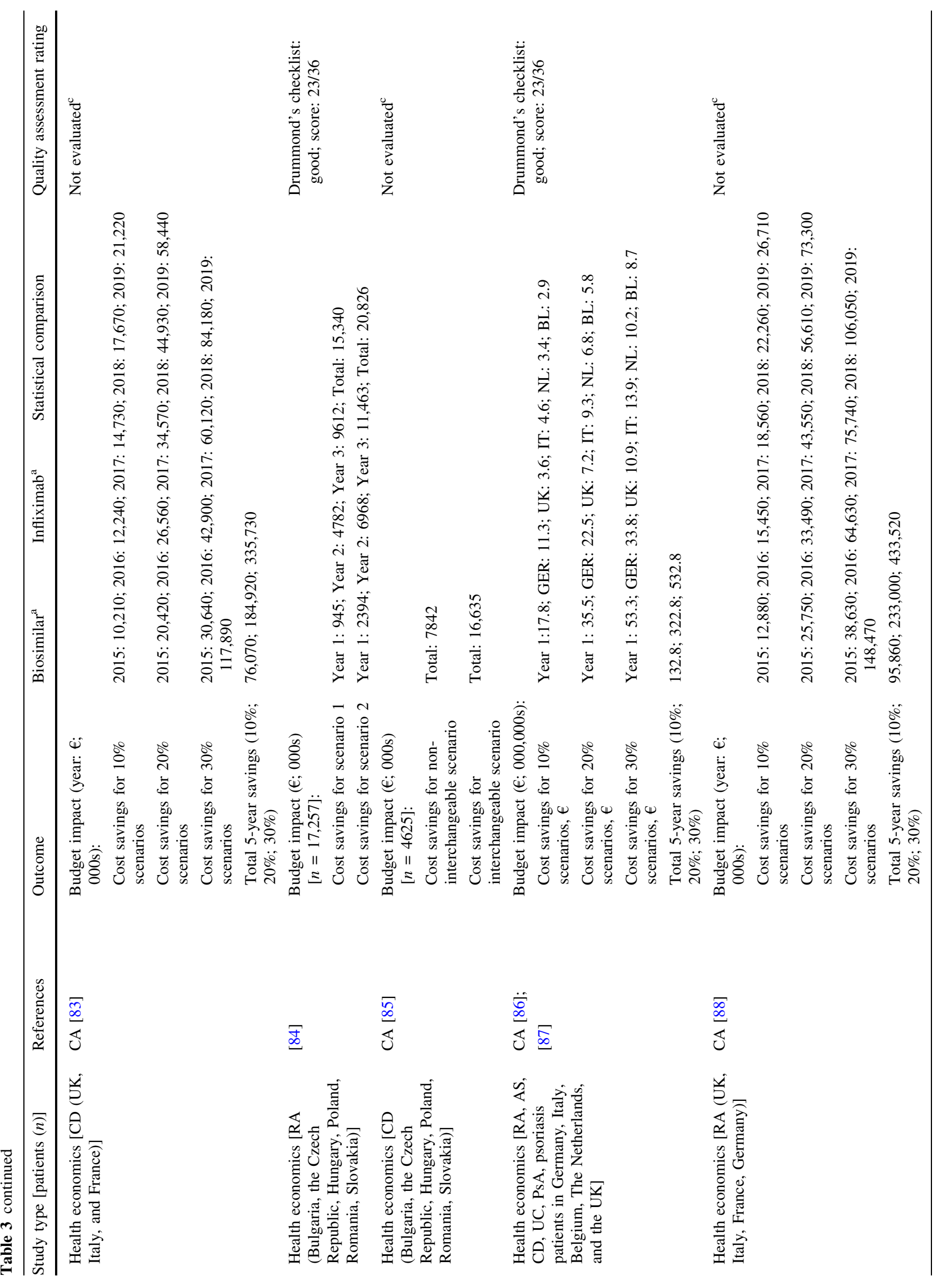




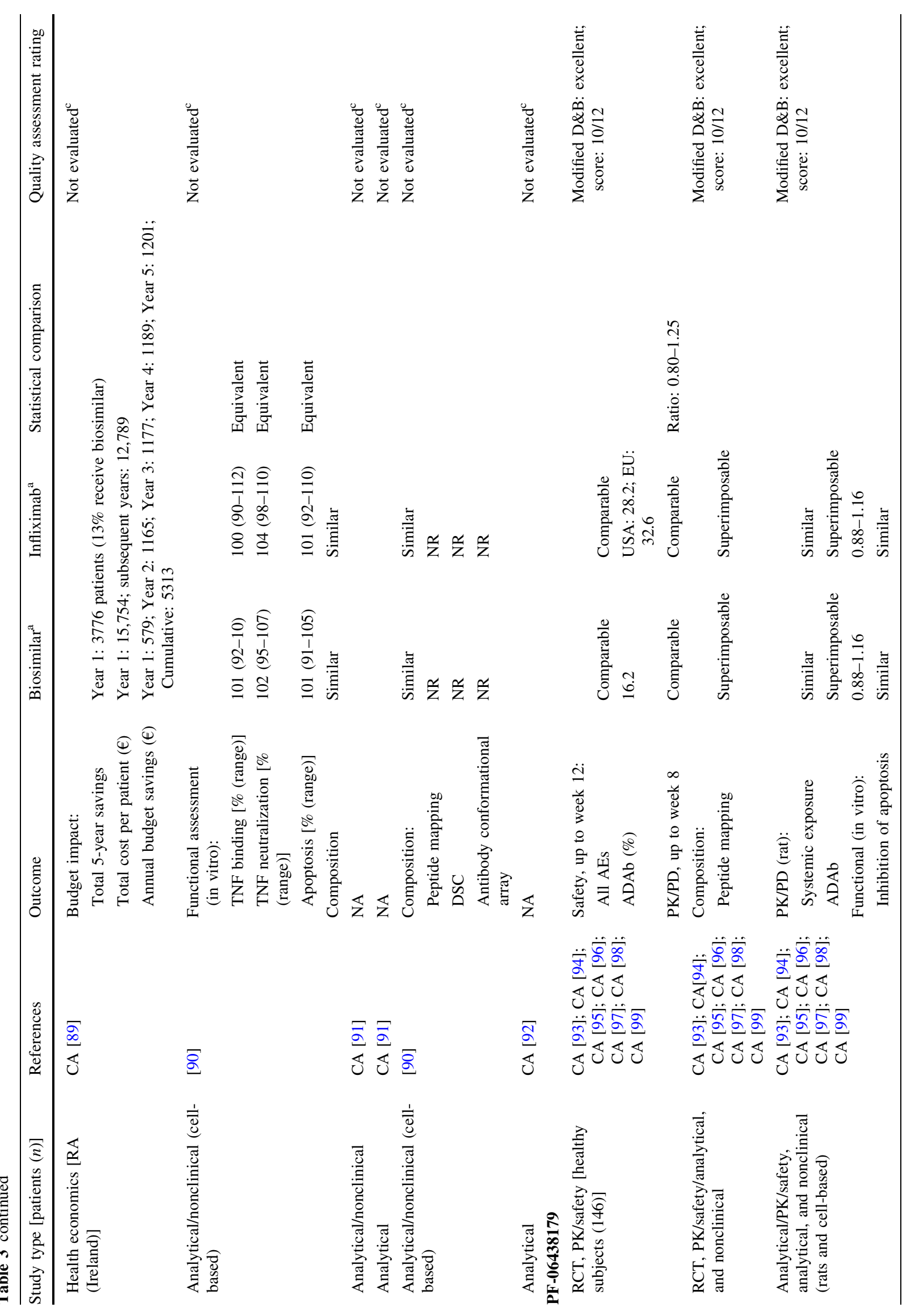




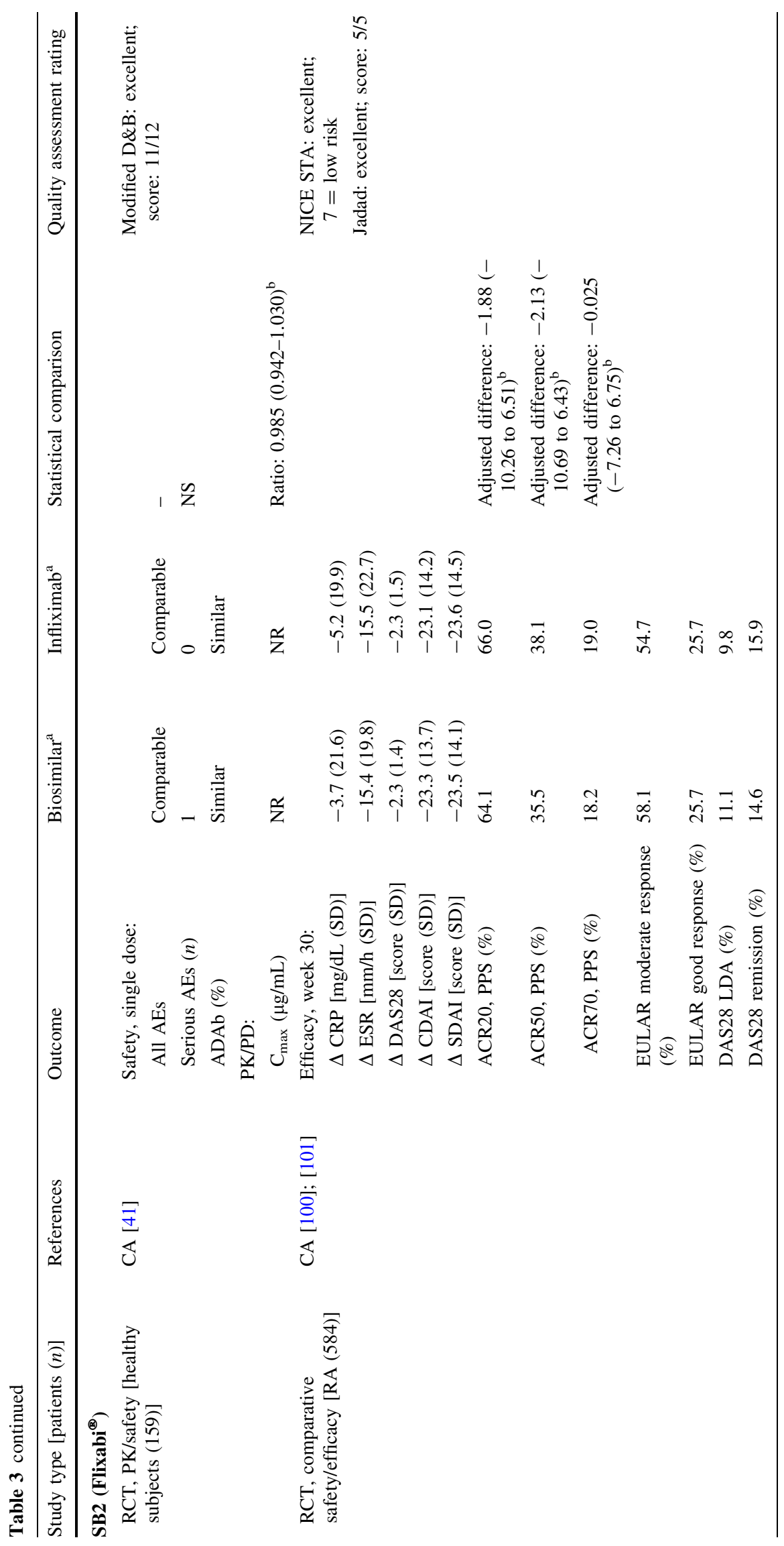




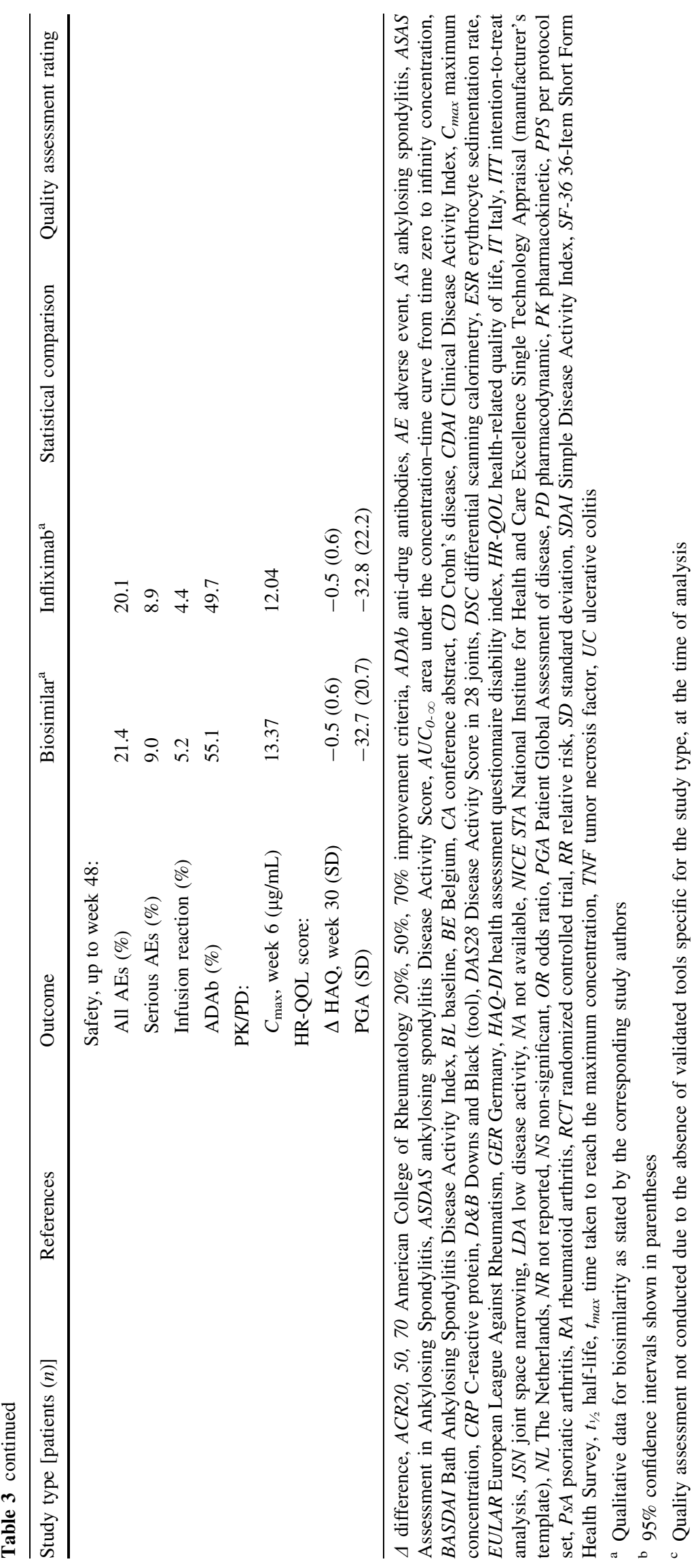


SB2 (Flixabi ${ }^{\circledR}$; Samsung Bioepis) The pharmacokinetics, safety, and immunogenicity profiles were reportedly similar between SB2 and infliximab in healthy volunteers [41]. No analytical or nonclinical studies comparing structural or functional similarity of SB2 versus infliximab had been published at the time of review.

CT-P13 (Remsima ${ }^{\circledR} /$ Inflectra ${ }^{\circledR}$; Celltrion) CT-P13 was evaluated in healthy human volunteers and demonstrated similar safety and pharmacokinetic profiles as those for infliximab [63]. The physicochemical properties of CT-P13 were also characterized using combined analytical techniques $[91,90]$. The results, overall, demonstrated that CTP13 has comparable structural properties to that of infliximab. A nonclinical cell-based study has also been reported, confirming bioequivalence in vitro.

\subsubsection{Rituximab Biosimilars}

Studies of rituximab biosimilars are presented in Table 4.

GP2013 (Sandoz) The functional similarity and pharmacokinetic properties of GP2013 compared with rituximab have been assessed both in vitro (using lymphoma cell lines) [117] and in vivo (mouse xenograft models and cynomolgus monkeys) $[117,115]$. The studies also included structural comparability assessments using a combination of biochemical techniques. These studies suggest high levels of similarity between GP2013 and the originator rituximab.

PF-05280586 (Pfizer) Comparative nonclinical studies have been published for PF-05280586 and rituximab, based on in vitro functional assessments and evaluation of safety or pharmacokinetic in vivo (monkeys) $[114,109,111,113,108$, 112]. In vitro data (complement-dependent cytotoxicity assays and biologic activity) provide evidence of functional similarity to the originator. The safety, tolerability, and pharmacokinetic/pharmacodynamic profile of PF-05280586 in monkeys is reported to be comparable to rituximab. These studies also included comprehensive analytical assessments of the primary and higher-order structure, and concluded high similarity between the biosimilar and the originator.

RTXM83 (Mabxience) The biological potency and pharmacokinetic/pharmacodynamic properties of RTXM83 have been evaluated in a single preclinical study (unspecified cell line or cynomolgus monkeys) [118]. The results of the study suggest functional similarity and analogous pharmacokinetic/pharmacodynamic properties between the biosimilar and originator.

\subsection{Clinical Evidence in Rheumatoid Arthritis (RA)}

\subsubsection{Adalimumab Biosimilars in RA}

Studies of adalimumab biosimilars are presented in Table 1.
Exemptia $^{T M}$ (Cadila Healthcare) The safety and efficacy of Exemptia ${ }^{\mathrm{TM}}$ versus adalimumab have been reported in a comparative safety/efficacy trial in patients $(n=120)$ with moderate-to-severe RA [33]. The results demonstrated no significant difference in any of the parameters (with respect to efficacy, tolerability, and safety) at the end of the study between the two treatment groups. No analytical or nonclinical study data evaluating the structural composition or functional similarity of Exemptia ${ }^{\mathrm{TM}}$ with respect to adalimumab were extracted during this literature review.

\subsubsection{Etanercept Biosimilars in RA}

Studies of etanercept biosimilars are presented in Table 2. HD2O3 (Davictrel ${ }^{T M}$; Hanwha Chemical) In a comparative safety/efficacy trial comparing HD203 with etanercept in patients with active RA $(n=233)$ and insufficient response to methotrexate, the proportion of patients achieving American College of Rheumatology (ACR) improvement of $20 \%$ (ACR20) at week 24 was not significantly different between HD203 and etanercept. Similar trends were seen for ACR improvement of 50\% (ACR50) and of 70\% (ACR70) [46, 45].

SB4 (Benepali ${ }^{\circledR}$; Samsung Bioepis) One comparative safety/efficacy RCT for SB4 was found for patients with RA $(n=596)$, which reported that SB4 was equivalent to etanercept in terms of efficacy and had a comparable safety profile [48, 49].

\subsubsection{Infliximab Biosimilars in RA}

Studies of infliximab biosimilars are presented in Table 3. BOW015 (Infimab ${ }^{\circledR} ;$ Ranbaxy Laboratories) Prior to discontinuation, BOW015 was investigated in a comparative safety/efficacy equivalence study in patients with RA $(n=189)$. Results of the study implied similarity with respect to efficacy (disease activity and physical function) and safety compared with infliximab [54, 119, 57, 56, 120].

SB2 (Flixabi ${ }^{\circledR}$; Samsung Bioepis) SB2 has been studied in a comparative safety/efficacy study to demonstrate equivalence of efficacy, safety, immunogenicity, and pharmacokinetic outcomes versus infliximab in patients with moderate to severe RA $(n=584)[100,101]$. SB2 demonstrated equivalent efficacy to infliximab in regards to ACR20 response. The pharmacokinetic, safety, and immunogenicity profiles were also reportedly similar to infliximab.

CT-P13 (Remsima $^{\circledR} /$ Inflectra $^{\circledR}$; Celltrion) In the PLANETRA (Programme evaLuating the Autoimmune disease iNvEstigational drug cT-p13 in RA patients) comparative safety/efficacy study, CT-P13 displayed equivalent efficacy to infliximab, was well-tolerated, and had a comparable pharmacokinetic, safety, and immunogenicity profile, as 
Table 4 Outcomes for rituximab biosimilars

\begin{tabular}{|c|c|c|c|c|c|c|}
\hline $\begin{array}{l}\text { Study type [patients } \\
(n)]\end{array}$ & Reference & Outcome & Biosimilar $^{\mathrm{a}}$ & Rituximab $^{\mathrm{a}}$ & $\begin{array}{l}\text { Statistical } \\
\text { comparison }\end{array}$ & Quality assessment rating \\
\hline \multicolumn{7}{|l|}{ CT-P10 } \\
\hline \multirow[t]{15}{*}{$\begin{array}{l}\text { RCT, PK/safety } \\
{[\text { RA (154)] }}\end{array}$} & \multirow[t]{15}{*}{ CA [102]; CA [103] } & $\begin{array}{l}\text { Efficacy, week } \\
24 \text { : }\end{array}$ & & & & \multirow[t]{15}{*}{$\begin{array}{l}\text { Modified D\&B: excellent; } \\
\text { score: } 9 / 12\end{array}$} \\
\hline & & ACR20 (\%) & 63.0 & 66.7 & & \\
\hline & & ACR50 (\%) & 37.0 & 31.3 & & \\
\hline & & $\operatorname{ACR} 70(\%)$ & 16.0 & 14.6 & & \\
\hline & & $\begin{array}{l}\text { Safety, week 24: } \\
\text { All AEs }(n)\end{array}$ & & & & \\
\hline & & Treatment AEs & 166 & 88 & & \\
\hline & & Infections $(\%)$ & 23.5 & 25.5 & & \\
\hline & & $\begin{array}{l}\text { Infusion } \\
\text { reactions (\%) }\end{array}$ & 16.7 & 19.6 & & \\
\hline & & $\begin{array}{l}\text { Serious AEs } \\
(\%)\end{array}$ & 16.7 & 17.6 & & \\
\hline & & $\begin{array}{l}\text { Serious AEs } \\
\text { RTX Abs +ve } \\
(\%)\end{array}$ & 11.1 & 22.2 & & \\
\hline & & $\begin{array}{l}\text { Serious AEs } \\
\text { RTX Abs -ve } \\
(\%)\end{array}$ & 15.6 & 16.2 & & \\
\hline & & $\mathrm{ADAb}(\%)$ & 17.6 & 17.6 & & \\
\hline & & $\begin{array}{c}\text { PK/PD, week 24: } \\
C_{\max }(\mu \mathrm{g} / \mathrm{mL})\end{array}$ & 465.9 & 477.5 & $\begin{array}{l}\text { Ratio: } 0.976 \\
\quad(0.92-1.035)^{b}\end{array}$ & \\
\hline & & $\begin{array}{l}\text { AUC ( } \mu \mathrm{g} \cdot \text { day/ } \\
\mathrm{mL})\end{array}$ & 7870.8 & 8010.4 & $\begin{array}{l}\text { Ratio: } 0.983 \\
\quad(0.896-1.078)^{\mathrm{b}}\end{array}$ & \\
\hline & & $\begin{array}{l}\text { AUC of B cell } \\
\text { count }(\text { cells } / \mu \mathrm{L})\end{array}$ & 20.8 & 20.4 & $\begin{array}{l}\text { Ratio: } 1.02 \\
\quad(0.98-1.06)^{\mathrm{b}}\end{array}$ & \\
\hline \multicolumn{7}{|l|}{ PF-05280586 } \\
\hline \multirow{11}{*}{$\begin{array}{l}\mathrm{RCT}, \mathrm{PK} / \text { safety, } \\
\text { preliminary } \\
\text { safety/efficacy } \\
{[\mathrm{RA}(220)]}\end{array}$} & \multirow[t]{11}{*}{$\begin{array}{l}\text { CA [104]; CA [105]; } \\
\text { CA [106]; CA [107] }\end{array}$} & $\begin{array}{l}\text { Efficacy, week } \\
\text { 16: }\end{array}$ & & & & \multirow[t]{11}{*}{$\begin{array}{l}\text { Modified D\&B: good; } \\
\text { score: } 7 / 12\end{array}$} \\
\hline & & $\begin{array}{l}\text { ACR20, 50, and } \\
70(\%)\end{array}$ & Improved & Improved & & \\
\hline & & DAS28-CRP & Improved & Improved & & \\
\hline & & CRP (mg/L) & Improved & Improved & & \\
\hline & & Safety, week 16: & & & & \\
\hline & & Serious AEs $(n)$ & 5 & 6 & & \\
\hline & & $\begin{array}{l}\text { PK/PD, week 16: } \\
C_{\max }(\mu \mathrm{g} / \mathrm{mL})\end{array}$ & Similar & Similar & & \\
\hline & & $\begin{array}{l}\text { AUC ( } \mu \mathrm{g} \cdot \text { day/ } \\
\mathrm{mL})\end{array}$ & Similar & Similar & & \\
\hline & & $t_{1 / 2}(\mathrm{~h})$ & Similar & Similar & & \\
\hline & & $\begin{array}{l}\text { Clearance (mL/ } \\
\mathrm{h} / \mathrm{kg})\end{array}$ & Similar & Similar & & \\
\hline & & $\begin{array}{l}\text { Volume of } \\
\text { distribution } \\
(\mathrm{mL} / \mathrm{kg})\end{array}$ & Similar & Similar & & \\
\hline
\end{tabular}


Table 4 continued

\begin{tabular}{|c|c|c|c|c|c|c|}
\hline $\begin{array}{l}\text { Study type [patients } \\
(n)]\end{array}$ & Reference & Outcome & Biosimilar $^{\mathrm{a}}$ & Rituximab $^{\mathrm{a}}$ & $\begin{array}{l}\text { Statistical } \\
\text { comparison }\end{array}$ & Quality assessment rating \\
\hline \multirow[t]{30}{*}{$\begin{array}{l}\text { Nonclinical } \\
\text { (cynomolgus } \\
\text { monkeys and cell- } \\
\text { based) }\end{array}$} & \multirow[t]{30}{*}{$\begin{array}{l}\text { [108]; CA } \\
{[109] ; \mathrm{CA}} \\
{[110]}\end{array}$} & $\begin{array}{l}\text { Functional assessment } \\
\text { (in vitro): } \\
\text { CDC }\end{array}$ & Similar & Similar & & \multirow[t]{30}{*}{$\begin{array}{l}\text { SYRCLE'S risk of bias: } \\
\text { moderate; unclear }=9 \text {, } \\
\text { low risk }=13\end{array}$} \\
\hline & & Safety (monkey): & Similar & Similar & & \\
\hline & & ADAb, single-dose $(\%)$ & 100 & 100 & & \\
\hline & & $\begin{array}{l}\text { ADAb, repeated-dose, day } \\
22(\%)\end{array}$ & 71 & 36 & & \\
\hline & & $\begin{array}{l}\text { ADAb, repeated-dose, day } \\
114(\%)\end{array}$ & 50 & 50 & & \\
\hline & & \multicolumn{4}{|l|}{ PK/PD (monkey): } & \\
\hline & & $\begin{array}{l}\text { AUC, } 2 \mathrm{mg} / \mathrm{kg}[\mu \mathrm{g} \cdot \mathrm{h} / \mathrm{mL} \\
(\mathrm{SD})]\end{array}$ & $\begin{array}{l}4720 \\
(966.0)\end{array}$ & $4940(890.0)$ & & \\
\hline & & $\begin{array}{l}\text { AUC, } 10 \mathrm{mg} / \mathrm{kg}[\mu \mathrm{g} \cdot \mathrm{h} / \mathrm{mL} \\
\text { (SD)] }\end{array}$ & $\begin{array}{l}34,700 \\
(8650.0)\end{array}$ & $37,100(6010$ & $0.0)$ & \\
\hline & & $\begin{array}{l}\text { AUC, } 20 \mathrm{mg} / \mathrm{kg}[\mu \mathrm{g} \cdot \mathrm{h} / \mathrm{mL} \\
\text { (SD)] }\end{array}$ & $\begin{array}{l}64,000 \\
(14,600.0)\end{array}$ & $51,700(11,9$ & $00.0)$ & \\
\hline & & $\begin{array}{l}\text { AUC, repeated-dose, day } 1 \\
{[\mu \mathrm{g} \cdot \mathrm{h} / \mathrm{mL}(\mathrm{SD})]}\end{array}$ & $\begin{array}{l}56,800 \\
(15,400.0)\end{array}$ & $54,600(8,80$ & $0.0)$ & \\
\hline & & $\begin{array}{l}\text { AUC, repeated-dose, day } 22 \\
{[\mu \mathrm{g} \cdot \mathrm{h} / \mathrm{mL}(\mathrm{SD})]}\end{array}$ & $\begin{array}{l}53,200 \\
(18,700.0)\end{array}$ & $79,500(39,9$ & $00.0)$ & \\
\hline & & $C_{\max }, 2 \mathrm{mg} / \mathrm{kg}[\mu \mathrm{g} / \mathrm{mL}(\mathrm{SD})]$ & $74.0(15.5)$ & $80.3(7.95)$ & & \\
\hline & & $\begin{array}{l}C_{\max }, 10 \mathrm{mg} / \mathrm{kg}[\mu \mathrm{g} / \mathrm{mL} \\
(\mathrm{SD})]\end{array}$ & $\begin{array}{l}481.0 \\
(70.4)\end{array}$ & $497.0(62.2)$ & & \\
\hline & & $\begin{array}{l}C_{\max }, 20 \mathrm{mg} / \mathrm{kg}[\mu \mathrm{g} / \mathrm{mL} \\
(\mathrm{SD})]\end{array}$ & $\begin{array}{l}912.0 \\
\quad(198.0)\end{array}$ & $726.0(138.0$ & & \\
\hline & & $\begin{array}{l}C_{\max }, \text { repeated-dose, day } 1 \\
{[\mu \mathrm{g} / \mathrm{mL}(\mathrm{SD})]}\end{array}$ & $\begin{array}{l}848.0 \\
\quad(241.0)\end{array}$ & $903.0(292.0$ & & \\
\hline & & $\begin{array}{l}C_{\max }, \text { repeated-dose, day } 22 \\
{[\mu \mathrm{g} / \mathrm{mL}(\mathrm{SD})]}\end{array}$ & $\begin{array}{l}966.0 \\
\quad(966.0)\end{array}$ & $1230.0(313$. & & \\
\hline & & $t_{1 / 2}, 2 \mathrm{mg} / \mathrm{kg}[\mathrm{h}(\mathrm{SD})]$ & $1.22(2.37)$ & $0.08(0.0)$ & & \\
\hline & & $t_{1 / 2}, 10 \mathrm{mg} / \mathrm{kg}[\mathrm{h}(\mathrm{SD})]$ & $0.24(0.37)$ & $0.24(0.37)$ & & \\
\hline & & $t_{1 / 2}, 20 \mathrm{mg} / \mathrm{kg}[\mathrm{h}(\mathrm{SD})]$ & $0.24(0.37)$ & $0.39(0.47)$ & & \\
\hline & & $t_{1 / 2}$, repeated-dose $[\mathrm{h}(\mathrm{SD})]$ & $0.70(1.57)$ & $0.35(0.43)$ & & \\
\hline & & $t_{1 / 2}$, repeated-dose $[\mathrm{h}(\mathrm{SD})]$ & $0.48(0.47)$ & $0.67(0.46)$ & & \\
\hline & & $t_{\max }$ & Similar & Similar & & \\
\hline & & $\begin{array}{l}\text { Reduction in splenic weight } \\
(\%)\end{array}$ & $12-42$ & $15-44$ & & \\
\hline & & \multicolumn{4}{|l|}{ Single-dose: } & \\
\hline & & $\begin{array}{l}\text { CD3-CD20+, } 2 \mathrm{mg} ; 10 \mathrm{mg} \text {; } \\
20 \mathrm{mg} \text {, day } 4(\%)\end{array}$ & $\begin{array}{l}99.3 ; 99.7 \\
99.2\end{array}$ & $98.4 ; 99.5 ; 9$ & 99.0 & \\
\hline & & $\begin{array}{l}\text { CD3-CD20+, } 2 \mathrm{mg} ; 10 \mathrm{mg} \text {; } \\
20 \mathrm{mg} \text {, day } 15(\%)\end{array}$ & $\begin{array}{l}92.1 ; 97.4 \\
98.7\end{array}$ & $89.5 ; 99.7 ; 9$ & 97.0 & \\
\hline & & $\begin{array}{l}\text { CD3-CD20+, } 2 \mathrm{mg} ; 10 \mathrm{mg} \text {; } \\
20 \mathrm{mg} \text {, day } 92(\%)\end{array}$ & $\begin{array}{l}27.7 ; 28.7 \\
35.1\end{array}$ & $27.8 ; 18.3 ; 2$ & 24.1 & \\
\hline & & $\begin{array}{l}\text { CD3-CD20+CD40+, } 2 \mathrm{mg} \\
10 \mathrm{mg} ; 20 \mathrm{mg} \text {, day } 4(\%)\end{array}$ & $\begin{array}{l}100 ; 100 \\
100\end{array}$ & $100 ; 100 ; 10$ & & \\
\hline & & $\begin{array}{l}\text { CD3-CD20+CD40+, } 2 \mathrm{mg} \\
10 \mathrm{mg} ; 20 \mathrm{mg} \text {, day } 15(\%)\end{array}$ & $\begin{array}{l}91.1 ; 97.0 \\
100\end{array}$ & $89.0 ; 99.2 ; 9$ & 95.0 & \\
\hline & & $\begin{array}{l}\text { CD3-CD } 20+C D 40+, 2 \mathrm{mg} \\
10 \mathrm{mg} ; 20 \mathrm{mg} \text {, day } 92(\%)\end{array}$ & $\begin{array}{l}29.1 ; 8.3 \\
27.5\end{array}$ & $40.0 ;-15.5$ & 18.8 & \\
\hline
\end{tabular}


Table 4 continued

\begin{tabular}{|c|c|c|c|c|c|c|}
\hline Study type [patients $(n)]$ & Reference & Outcome & Biosimilar $^{\mathrm{a}}$ & Rituximab $^{\mathrm{a}}$ & $\begin{array}{l}\text { Statistical } \\
\text { comparison }\end{array}$ & $\begin{array}{l}\text { Quality assessment } \\
\text { rating }\end{array}$ \\
\hline & & $\begin{array}{l}\text { CD3-CD19+, } 2 \mathrm{mg} \\
10 \mathrm{mg} ; 20 \mathrm{mg} \text {, day } 4(\%)\end{array}$ & $86.3 ; 90.2 ; 90.5$ & $83.5 ; 85.2 ; \varepsilon$ & & \\
\hline & & $\begin{array}{l}\text { CD3-CD19+, } 2 \mathrm{mg} ; \\
10 \mathrm{mg} ; 20 \mathrm{mg} \text {, day } 15(\%)\end{array}$ & $81.0 ; 85.6 ; 91.1$ & $74.6 ; 88.7 ; \varepsilon$ & & \\
\hline & & $\begin{array}{l}\text { CD3-CD19+, } 2 \mathrm{mg} ; \\
10 \mathrm{mg} ; 20 \mathrm{mg} \text {, day } 92(\%)\end{array}$ & $9.8 ; 2.1 ; 29.6$ & $6.2 ;-5.2 ;-8$ & & \\
\hline & & $\begin{array}{l}\text { CD3-CD } 40+, 22 \mathrm{mg} ; \\
10 \mathrm{mg} ; 20 \mathrm{mg} \text {, day } 4(\%)\end{array}$ & $85.9 ; 91.4 ; 92.6$ & $88.0 ; 87.3 ; \varepsilon$ & & \\
\hline & & $\begin{array}{l}\text { CD3-CD40+, } 2 \mathrm{mg} ; \\
10 \mathrm{mg} ; 20 \mathrm{mg} \text {, day } 15(\%)\end{array}$ & $81.0 ; 84.8 ; 94.9$ & $78.4 ; 88.2 ; \varepsilon$ & & \\
\hline & & $\begin{array}{l}\text { CD3-CD40+, } 2 \mathrm{mg} ; \\
10 \mathrm{mg} ; 20 \mathrm{mg} \text {, day } 92(\%)\end{array}$ & $25.3 ; 1.5 ; 18.7$ & $25.5 ;-30.0$ & & \\
\hline & & Repeated-dose: & & & & \\
\hline & & $\begin{array}{l}\text { CD3-CD20+, } 2 \mathrm{mg} ; \\
10 \mathrm{mg} ; 20 \mathrm{mg} \text {, day } 4(\%)\end{array}$ & 100 & 100 & & \\
\hline & & $\begin{array}{l}\text { CD3-CD20+, } 2 \mathrm{mg} ; \\
10 \mathrm{mg} ; 20 \mathrm{mg} \text {, day } 15(\%)\end{array}$ & 99.9 & 99.7 & & \\
\hline & & $\begin{array}{l}\text { CD3-CD20+, } 2 \text { mg; } \\
10 \mathrm{mg} ; 20 \mathrm{mg} \text {, day } 121 \\
(\%)\end{array}$ & 75.5 & 80.9 & & \\
\hline & & $\begin{array}{l}\text { CD3-CD20+CD } 40+, \\
2 \mathrm{mg} ; 10 \mathrm{mg} ; 20 \mathrm{mg} \text {, day } \\
4(\%)\end{array}$ & 100 & 90.1 & & \\
\hline & & $\begin{array}{l}\text { CD3-CD20+CD40+, } \\
2 \mathrm{mg} ; 10 \mathrm{mg} ; 20 \mathrm{mg} \text {, day } \\
15(\%)\end{array}$ & 99.9 & 99.4 & & \\
\hline & & $\begin{array}{l}\text { CD3-CD20+CD } 40+, \\
2 \mathrm{mg} ; 10 \mathrm{mg} ; 20 \mathrm{mg} \text {, day } \\
121(\%)\end{array}$ & 76.1 & 80.5 & & \\
\hline & & $\begin{array}{l}\text { CD3-CD19+, } 2 \mathrm{mg} ; \\
10 \mathrm{mg} ; 20 \mathrm{mg} \text {, day } 4(\%)\end{array}$ & 96.1 & 92.1 & & \\
\hline & & $\begin{array}{l}\text { CD3-CD19+, } 2 \mathrm{mg} ; \\
10 \mathrm{mg} ; 20 \mathrm{mg} \text {, day } 15(\%)\end{array}$ & 90.3 & 89.6 & - & \\
\hline & & $\begin{array}{l}\text { CD3-CD19+, } 2 \mathrm{mg} ; \\
10 \mathrm{mg} ; 20 \mathrm{mg} \text {, day } 121 \\
(\%)\end{array}$ & 80.1 & 84.0 & - & \\
\hline & & $\begin{array}{l}\text { CD3-CD40+, } 2 \mathrm{mg} ; \\
10 \mathrm{mg} ; 20 \mathrm{mg} \text {, day } 4(\%)\end{array}$ & 97.3 & 86.0 & - & \\
\hline & & $\begin{array}{l}\text { CD3-CD40+, } 2 \mathrm{mg} ; \\
10 \mathrm{mg} ; 20 \mathrm{mg} \text {, day } 15(\%)\end{array}$ & 97.3 & 96.8 & & \\
\hline & & $\begin{array}{l}\text { CD3-CD40+, } 2 \mathrm{mg} ; \\
10 \mathrm{mg} ; 20 \mathrm{mg} \text {, day } 121 \\
(\%)\end{array}$ & 74.3 & 77.1 & & \\
\hline \multirow[t]{2}{*}{ Nonclinical (cell-based) } & CA [111] & $\begin{array}{l}\text { Functional assessment } \\
\text { (in vitro): }\end{array}$ & & & & Not evaluated ${ }^{\mathrm{d}}$ \\
\hline & & Biologic activity (\%) & $93-114$ & USA/EU: $7 C$ & -135 & \\
\hline \multirow[t]{4}{*}{ Nonclinical (cell-based) } & CA [112] & $\begin{array}{l}\text { Functional assessment } \\
\text { (in vitro): }\end{array}$ & & & & Not evaluated $^{\mathrm{d}}$ \\
\hline & & $\begin{array}{l}\text { ADCC dose-response } \\
\text { curve }\end{array}$ & Superimposable & \multicolumn{2}{|c|}{ Superimposable } & \\
\hline & & CDC dose-response curve & Superimposable & \multicolumn{2}{|c|}{ Superimposable } & \\
\hline & & Binding to Fc $\gamma$ RIIIa & Similar & \multicolumn{2}{|l|}{ Similar } & \\
\hline
\end{tabular}


Table 4 continued

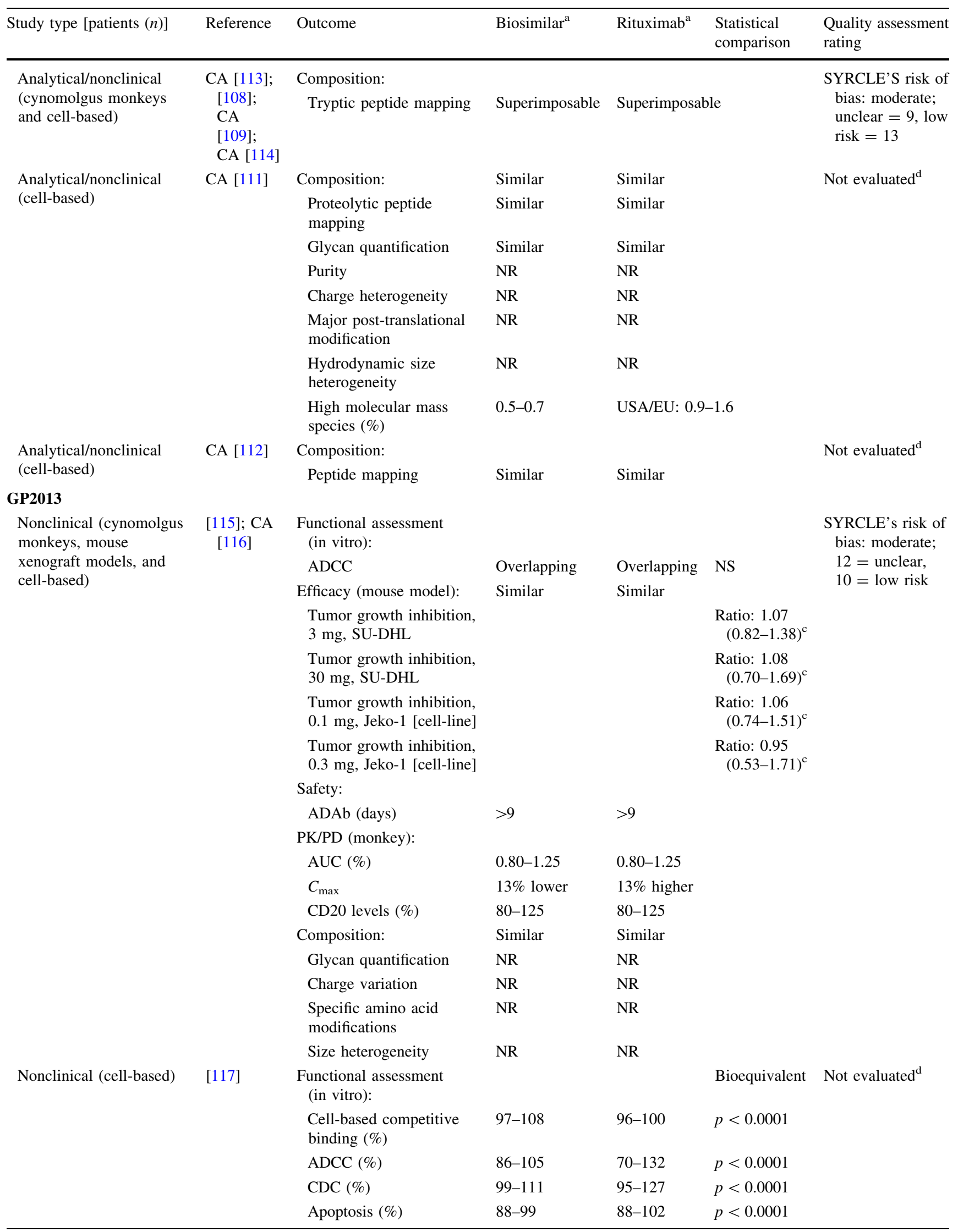


Table 4 continued

\begin{tabular}{|c|c|c|c|c|c|c|}
\hline Study type [patients $(n)]$ & Reference & Outcome & Biosimilar $^{\mathrm{a}}$ & Rituximab $^{\mathrm{a}}$ & $\begin{array}{l}\text { Statistical } \\
\text { comparison }\end{array}$ & $\begin{array}{l}\text { Quality assessment } \\
\text { rating }\end{array}$ \\
\hline \multirow{5}{*}{$\begin{array}{l}\text { Analytical (cynomolgus } \\
\text { monkeys, mouse models, } \\
\text { and cell-based) }\end{array}$} & \multirow{5}{*}{$\begin{array}{l}{[115]} \\
\text { CA [116] }\end{array}$} & Composition: & Similar & Similar & & \multirow{5}{*}{$\begin{array}{l}\text { SYRCLE's risk of } \\
\text { bias: moderate; } \\
12=\text { unclear, } \\
10=\text { low risk }\end{array}$} \\
\hline & & Glycan quantification & NR & NR & & \\
\hline & & Charge variation & NR & NR & & \\
\hline & & $\begin{array}{l}\text { Specific amino acid } \\
\text { modifications }\end{array}$ & NR & NR & & \\
\hline & & Size heterogeneity & NR & NR & & \\
\hline \multirow{9}{*}{$\begin{array}{l}\text { Analytical/nonclinical } \\
\text { (cell-based) }\end{array}$} & \multirow[t]{9}{*}{ [117] } & Composition: & & & & \multirow[t]{10}{*}{ Not evaluated $^{\mathrm{d}}$} \\
\hline & & Primary structure & Identical & Identical & & \\
\hline & & Higher order structure & $\begin{array}{c}\text { Expected } \\
\text { pattern }\end{array}$ & & & \\
\hline & & Stability & Superimposable & & & \\
\hline & & Free thiol analysis & Comparable & & & \\
\hline & & $\begin{array}{l}\text { Deamidation, deamidated } \\
\text { L28H [peptide] (\%) }\end{array}$ & 0.5 & 1.0 & & \\
\hline & & Glycation (\%) & $2-3$ & $2-3$ & & \\
\hline & & $\begin{array}{l}\text { Glycosylation site } \\
\text { analysis }\end{array}$ & Similar & & & \\
\hline & & $\begin{array}{l}\text { Purity, aggregate and } \\
\text { particle levels }\end{array}$ & Similar & & & \\
\hline \multicolumn{6}{|l|}{ RTXM83 } & \\
\hline \multirow[t]{8}{*}{$\begin{array}{l}\text { Nonclinical (cynomolgus } \\
\text { monkeys and cell-based) }\end{array}$} & \multirow[t]{8}{*}{ CA [118] } & $\begin{array}{l}\text { Functional assessment } \\
\text { (in vitro): }\end{array}$ & & & & \multirow[t]{8}{*}{ Not evaluated ${ }^{\mathrm{d}}$} \\
\hline & & ADCC, binding & Similar & Similar & & \\
\hline & & CDC, potency & Similar & Similar & & \\
\hline & & PK/PD (monkey): & & & & \\
\hline & & $\operatorname{AUC}(\%)$ & $80-120$ & & & \\
\hline & & $C_{\max }(\%)$ & $80-120$ & & & \\
\hline & & $t_{1 / 2}$, repeated-dose $(\%)$ & $80-120$ & & & \\
\hline & & $\begin{array}{l}\text { CD20 and CD40 } \\
\text { depletion }\end{array}$ & Similar & Similar & & \\
\hline \multirow{3}{*}{$\begin{array}{l}\text { Analytical/nonclinical } \\
\text { (cell-based) }\end{array}$} & \multirow[t]{3}{*}{ CA [118] } & Composition: & & & & \multirow[t]{3}{*}{ Not evaluated ${ }^{\mathrm{d}}$} \\
\hline & & Peptide mapping & Similar & Similar & & \\
\hline & & $\begin{array}{l}\text { Glycan quantification and } \\
\text { charge variant }\end{array}$ & Similar & Similar & & \\
\hline
\end{tabular}

Abs antibodies, ACR20, 50, 70 American College of Rheumatology 20, 50, 70\% improvement criteria, $A D A b$ anti-drug antibodies, $A D C C$ antibody-dependent cell-mediated cytotoxicity, $A E$ adverse event, $A U C$ area under the plasma concentration-time curve, $C A$ conference abstract, $C D C$ complement-dependent cytotoxicity, $C_{\max }$ maximum concentration, $C R P$ C-reactive protein, $D \& B$ Downs and Black (tool), DAS28 Disease Activity Score in 28 joints, $F c \gamma R I I I a$ Fc gamma receptor IIIa, NR not reported, $N S$ not significant, $P D$ pharmacodynamic, $P K$ pharmacokinetic, $R A$ rheumatoid arthritis, $R C T$ randomized controlled trial, $R T X$ rituximab, $S D$ standard deviation, $S U-D H L$ Southwestern University Diffuse Histiocytic Lymphoma, SYRCLE SYstematic Review Centre for Laboratory animal Experimentation, $t_{1 / 2}$ half-life, $t_{\max }$ time to maximum concentration, +ve positive, -ve negative

${ }^{a}$ Qualitative data for biosimilarity as stated by the corresponding study authors

b $90 \%$ confidence intervals shown in parentheses

c $95 \%$ confidence intervals shown in parentheses

${ }^{\mathrm{d}}$ Quality assessment not conducted due to the absence of validated tools specific for the study type, at the time of analysis

compared with infliximab in patients with RA $(n=606)$ $[66,121,67,72,69,102,71]$. An observational crosssectional study, which included RA patients from Romania who were treated with biologic agents, assessed efficacy and safety data after 2 years of treatment with infliximab, CT-P13, etanercept, adalimumab, or rituximab [122]. 


\subsubsection{Rituximab Biosimilars in RA}

Studies of rituximab biosimilars are presented in Table 4.

CT-P10 (Celltrion) CT-P10 was evaluated in a pharmacokinetic/safety trial in patients with active RA and an inadequate response or intolerance to prior anti-TNF agents $(n=154)[102,103]$. The published findings of the study concluded equivalence in terms of efficacy, safety, and pharmacokinetic/pharmacodynamic outcomes compared with rituximab. There was some indication, however, that antidrug antibodies-positive patients in the rituximab group may have been at greater risk of serious AEs than those in the CT-P10 group.

PF-05280586 (Pfizer) Data from a pharmacokinetic/ safety study on PF-05280586 in patients with active RA on methotrexate and with prior inadequate response to antiTNF therapies $(n=220)$ also inferred equivalence of efficacy, safety, and pharmacokinetic/pharmacodynamic performance compared with rituximab.

\subsection{Clinical Evidence in Ankylosing Spondylitis} (AS)

\subsubsection{Infliximab Biosimilars in AS}

Studies of infliximab biosimilars are presented in Table 3. CT-P13 (Remsima $^{\circledR} /$ Inflectra $^{\circledR}$; Celltrion) CT-P13 has been evaluated in a pharmacokinetic/safety study (PLANETAS) study [63, 59, 58, 61, 123, 62]. The pharmacokinetic profiles of CT-P13 and infliximab were equivalent in patients with active AS $(n=250)$. CT-P13 was well-tolerated, with an efficacy and safety profile comparable with that of infliximab over the duration of the study.

\subsection{Clinical Evidence in Ulcerative Colitis (UC) and Crohn's Disease (CD)}

\subsubsection{Infliximab Biosimilars in $U C / C D$}

Studies of infliximab biosimilars are presented in Table 3.

CT-P13 (Remsima $^{\circledR} /$ Inflectra ${ }^{\circledR} ; \quad$ Celltrion) Several observational (post-marketing) studies have been reported for CT-P13 that investigated the efficacy and impact of switching from the biologic originator to CT-P13 in patients with UC or CD $(n=336)$ $[80,81,76,77,78,74,79,75,82]$. In one study of 110 patients with $\mathrm{CD}$ or UC, the clinical response to the treatment at 8 weeks was $87 \%$ in patients who had not been previously treated with an anti-TNF agent and $67 \%$ in those who had switched from another anti-TNF [76]. Another prospective study in 90 patients with $\mathrm{CD}$ or UC found decreases in scores of disease activity after 6 weeks of treatment with the biosimilar [81]. One of these observational cohort studies explored the use of CT-P13 in pediatric patients with $\mathrm{CD}(n=32)$ [82]. The results of these studies should be interpreted with a degree of caution based on the underlying limitations of the study designs.

\subsection{Health Economics Evidence: Infliximab Biosimilars}

Studies of infliximab biosimilars are presented in Table 3. CT-P13 (Remsima ${ }^{\circledR} /$ Inflectra ${ }^{\circledR}$; Celltrion) Following the launch of CT-P13 in several major European countries, health economic studies have emerged evaluating the budget impact and cost effectiveness (from a payer or patient perspective) of introducing the biosimilar into the chronic inflammatory disease market $[84,85,86,87$, 88, 83, 89]. Published studies to date have explored the potential cost savings realized from substituting the originator with CT-P13 for the treatment of CD, UC, RA, AS, and PsA across multiple European countries. The totality of evidence from these studies points towards substantial cost savings, with the degree of budget impact dependent on the rate of interchangeability, patient number, and eligibility of treatment with the biosimilar (i.e., whether patients are treatment-naïve or treatment-experienced), along with the acquisition cost of the biosimilar. These authors have attempted to quantitatively demonstrate that the introduction of a biosimilar may provide additional budget to treat more patients (including, for example, those with earlierstage disease) on an annual basis, which could potentially alleviate both the short- and longer-term cost burden among healthcare payers and providers.

\subsection{Preclinical and Clinical Data on Intended Copies}

\subsubsection{Intended Copies of Etanercept}

Studies of etanercept intended copies are presented in Table 5.

Infinitam $^{\circledR}$ (Probiomed) One preliminary safety/efficacy RCT for Infinitam ${ }^{\circledR}$ in patients with moderate to severe RA ( $n=58)$ was identified [124]. The results suggested similar efficacy, safety, and pharmacokinetic/pharmacodynamic outcomes between Infinitam ${ }^{\circledR}$ and etanercept at 24 weeks. An observational study investigating Infinitam ${ }^{\circledR}$, Yisaipu ${ }^{\circledR}$ (etanercept intended copy), or Kikuzubam ${ }^{\circledR}$ (rituximab intended copy) in patients with rheumatic diseases reported that a large proportion of patients experienced AEs, with more than one-third of AEs reportedly occurring on the same day as first treatment [125].

Yisaipu $^{\circledR}$ (Shanghai CP Guojian Pharmaceutical Co.) An observational retrospective cohort study was performed 
Table 5 Outcomes for etanercept intended copies

\begin{tabular}{|c|c|c|c|c|c|c|}
\hline Study type [patients $(n)]$ & Reference & Outcome & $\begin{array}{l}\text { Intended } \\
\text { copy }^{\mathrm{a}}\end{array}$ & Etanercept $^{\mathrm{a}}$ & $\begin{array}{l}\text { Statistical } \\
\text { comparison }\end{array}$ & $\begin{array}{l}\text { Quality assessment } \\
\text { rating }\end{array}$ \\
\hline \multicolumn{7}{|l|}{ Infinitam $^{\circledR}$} \\
\hline $\begin{array}{l}\text { RCT, preliminary } \\
\text { safety/efficacy study } \\
\text { [moderate and severe RA } \\
(58)]\end{array}$ & CA [124] & $\begin{array}{l}\text { Efficacy, week 24: } \\
\text { DAS28 (score) } \\
\text { Safety } \\
\text { PK/PD }\end{array}$ & $\begin{array}{l}2.8 \\
\text { Similar } \\
\text { NR }\end{array}$ & $\begin{array}{l}2.4 \\
\text { Similar } \\
\text { NR }\end{array}$ & $p=0.355$ & $\begin{array}{l}\text { Modified D\&B: } \\
\text { excellent; score: } \\
9 / 12\end{array}$ \\
\hline \multirow[t]{2}{*}{$\begin{array}{l}\text { Observational [rheumatic } \\
\text { diseases (219)] }\end{array}$} & \multirow[t]{2}{*}{ CA [125] } & $\begin{array}{l}\text { Safety: } \\
\text { All AEs } \\
\left(\text { Infinitam }{ }^{\circledR} /\right. \\
\text { Yisaipu }{ }^{\circledR} \text { ) }\end{array}$ & $\begin{array}{c}n=10 \text { of } \\
14\end{array}$ & NA & & \multirow[t]{2}{*}{$\begin{array}{l}\text { Modified D\&B: fair; } \\
\text { score: } 4 / 12\end{array}$} \\
\hline & & $\begin{array}{l}\text { All AEs } \\
\left(\text { Kikuzubam }^{\circledR}\right)\end{array}$ & $\begin{array}{r}n=101 \\
\text { of } 205\end{array}$ & NA & & \\
\hline \multicolumn{7}{|l|}{ Yisaipu ${ }^{\circledR}$} \\
\hline Observational [RA (802)] & CA [48] & NA & & & & $\begin{array}{l}\text { Modified D\&B: good; } \\
\text { score: } 5 / 12\end{array}$ \\
\hline \multirow[t]{7}{*}{ Observational [RA (158)] } & \multirow[t]{7}{*}{ CA [126] } & Efficacy: & & $\begin{array}{c}\text { [Study did not compare } \\
\text { etanercept originator] }\end{array}$ & & \multirow[t]{7}{*}{$\begin{array}{l}\text { Modified D\&B: good; } \\
\text { score: } 7 / 12\end{array}$} \\
\hline & & $\begin{array}{l}\text { DAS28 low score, } \\
\text { BL }(\%)\end{array}$ & 11.2 & ADA: 13.1; IFX: 8.5 & NS & \\
\hline & & $\begin{array}{l}\text { DAS28 low score, } \\
\text { week } 104(\%)\end{array}$ & 16.1 & ADA: 11.4 ; IFX: 17.1 & NS & \\
\hline & & $\begin{array}{l}\text { DAS28 moderate } \\
\text { score, BL }(\%)\end{array}$ & 51.6 & ADA: 45.9; IFX: 34.2 & NS & \\
\hline & & $\begin{array}{l}\text { DAS28 moderate } \\
\text { score, week } 104(\%)\end{array}$ & 9.6 & ADA: 11.4; IFX: 14.2 & NS & \\
\hline & & $\begin{array}{l}\text { DAS28 high score, } \\
\text { BL }(\%)\end{array}$ & 9.6 & ADA: 8.2 ; IFX: 28.5 & NS & \\
\hline & & $\begin{array}{l}\text { DAS28 high score, } \\
\text { week } 104(\%)\end{array}$ & 6.4 & ADA: 4.9 ; IFX: 0 & NS & \\
\hline \multirow[t]{5}{*}{$\begin{array}{l}\text { Health economics } \\
\text { [RA (China)] }\end{array}$} & \multirow[t]{5}{*}[127]{} & Costs: & $\begin{array}{l}{[\text { Strategy }} \\
9]^{\mathrm{b}}\end{array}$ & [vs. MTX, Strategy 1] & & \multirow{5}{*}{$\begin{array}{l}\text { Drummond's } \\
\text { checklist: good; } \\
\text { score: } 29 / 36\end{array}$} \\
\hline & & $\begin{array}{l}\text { Lifetime costs } \\
\text { (US\$) }\end{array}$ & 18,574 & $12,735.4$ & & \\
\hline & & LYs gained (years) & 23.50 & 23.5 & & \\
\hline & & $\begin{array}{l}\text { QALYs gained } \\
\text { (years) }\end{array}$ & 9.76 & 9.1 & & \\
\hline & & ICERs & 8680 & & & \\
\hline
\end{tabular}

$A D A$ adalimumab, $A E$ adverse event, $B L$ baseline, $C A$ conference abstract, $D \& B$ Downs and Black (tool), DAS28 Disease Activity Score in 28 joints, ICER incremental cost-effectiveness ratio, $I F X$ infliximab, $L Y$ life-year, $M T X$ methotrexate, $N A$ not available, $N R$ not reported, $N S$ nonsignificant, $P D$ pharmacodynamic, $P K$ pharmacokinetic, $Q A L Y$ quality-adjusted life-year, $R A$ rheumatoid arthritis, $R C T$ randomized controlled trial

${ }^{a}$ Qualitative data for biosimilarity as stated by the corresponding study authors

b Nine strategies evaluated. Most cost-effective strategy is shown

in 158 patients with active RA treated with Yisaipu ${ }^{\circledR}$ (etanercept intended copy) $(n=62)$, adalimumab $(n=61)$, or infliximab $(n=35)$ [126]. Similar decreases in Disease Activity Score in 28 joints (DAS28) were seen at 24 months. No statistically significant differences were seen in the number of patients achieving remission. The authors noted that fewer AEs were present in the Yisaipu ${ }^{\circledR}$ treatment group than in the adalimumab- and infliximab- treated patients. Only one health economics study was identified for etanercept biosimilars/intended copies [127]. This study assessed the cost effectiveness of reduced doses or discontinuation of Yisaipu ${ }^{\circledR}$ in patients with moderately active RA. Strategies starting with Yisaipu ${ }^{\circledR} 50 \mathrm{mg} /$ week for 9 months followed by Yisaipu ${ }^{\circledR} 25$ or $50 \mathrm{mg} /$ week maintenance showed the greatest number of quality-adjusted life-years (QALYs) gained. The incremental cost- 
Table 6 Outcomes for rituximab intended copies

\begin{tabular}{|c|c|c|c|c|c|c|}
\hline Study type [patients $(n)$ ] & Reference & Outcome & Intended copy ${ }^{\mathrm{a}}$ & Rituximab $^{\mathrm{a}}$ & $\begin{array}{l}\text { Statistical } \\
\text { comparison }\end{array}$ & $\begin{array}{l}\text { Quality assessment } \\
\text { rating }\end{array}$ \\
\hline \multicolumn{7}{|l|}{ Reditux $^{\text {TM }}$} \\
\hline \multirow{5}{*}{$\begin{array}{l}\text { Observational/retrospective } \\
\text { [RA and failed DMARD } \\
\text { therapy (39)] }\end{array}$} & \multirow[t]{5}{*}{$\begin{array}{l}\text { CA [128]; } \\
\text { CA [129] }\end{array}$} & $\begin{array}{l}\text { Efficacy, week 24: } \\
\text { DAS-CRP }\end{array}$ & NR & NA & $p<0.0001$ & \multirow[t]{5}{*}{$\begin{array}{l}\text { Modified D\&B: } \\
\text { good; score: } 5 / 12\end{array}$} \\
\hline & & DAS-ESR & NR & NA & $p<0.0001$ & \\
\hline & & ACR20 (\%) & 97 & NA & & \\
\hline & & Safety, week 24: & & & & \\
\hline & & All SAEs $(n)$ & 0 & & & \\
\hline \multirow{11}{*}{$\begin{array}{l}\text { Observational/ prospective } \\
\text { [diffuse large B cell } \\
\text { lymphoma, RA, } \\
\text { scleroderma, } \\
\text { dermatomyositis (133)] }\end{array}$} & \multirow[t]{11}{*}{ CA [130] } & Safety: & & & & \multirow{11}{*}{$\begin{array}{l}\text { Modified D\&B: fair; } \\
\text { score: } 4 / 12\end{array}$} \\
\hline & & All AEs (\%) & 14.3 & NA & & \\
\hline & & Chills $(\%)$ & 20 & NA & & \\
\hline & & Headache $(\%)$ & 16.7 & NA & & \\
\hline & & Fever $(\%)$ & 13.0 & NA & & \\
\hline & & Urticaria (\%) & 10.0 & NA & & \\
\hline & & $\begin{array}{l}\text { All treatment-related AEs } \\
(\%)\end{array}$ & 73.0 & NA & & \\
\hline & & Mild AEs (\%) & 90.0 & NA & & \\
\hline & & Moderate AEs (\%) & 6.7 & NA & & \\
\hline & & Severe AEs (\%) & 3.3 & NA & & \\
\hline & & Mortality (\%) & 0 & NA & & \\
\hline \multirow{9}{*}{$\begin{array}{l}\text { Observational/prospective } \\
\text { [biologic-naïve RA (21)] }\end{array}$} & \multirow[t]{9}{*}{ [131] } & Efficacy: & & & & \multirow{9}{*}{$\begin{array}{l}\text { D\&B: good; score: } \\
\text { 11/26 }\end{array}$} \\
\hline & & DAS28-ESR score & $\begin{array}{l}2.54, p< \\
0.0001 \text { vs. } \\
\text { BL }\end{array}$ & NA & & \\
\hline & & DAS28 LDA (year 1) (\%) & 33 & NA & & \\
\hline & & DAS28 LDA (year 3) (\%) & 43 & NA & & \\
\hline & & $\begin{array}{l}\text { DAS28 remission (year 1) } \\
(\%)\end{array}$ & 57 & NA & & \\
\hline & & $\begin{array}{l}\text { DAS28 remission (year 3) } \\
(\%)\end{array}$ & 47 & NA & & \\
\hline & & Safety: & & & & \\
\hline & & Serious AEs $(n)$ & 0 & NA & & \\
\hline & & Infusion reactions (\%) & 10 & NA & & \\
\hline \multirow[t]{3}{*}{ Nonclinical (cell-based) } & \multirow[t]{3}{*}{ [132] } & $\begin{array}{l}\text { Functional assessment } \\
\text { (in vitro): }\end{array}$ & & & & \multirow[t]{3}{*}{ Not evaluated ${ }^{\mathrm{b}}$} \\
\hline & & $\operatorname{ADCC}(\%)$ & $80-125$ & $80-125$ & NS & \\
\hline & & CDC (3 batches) (\%) & $81 ; 111 ; 108$ & NR & & \\
\hline \multirow[t]{2}{*}{ Nonclinical (cell-based) } & \multirow[t]{2}{*}{ CA [133] } & $\begin{array}{l}\text { Safety (rat and rabbit cell- } \\
\text { lines): }\end{array}$ & & & & \multirow[t]{2}{*}{ Not evaluated ${ }^{\mathrm{b}}$} \\
\hline & & ADAb & Comparable & $\begin{array}{r}\text { Comparable } \\
\text { (USA/EU) }\end{array}$ & & \\
\hline \multirow{10}{*}{$\begin{array}{l}\text { Analytical/nonclinical (cell- } \\
\text { based) }\end{array}$} & \multirow[t]{10}{*}{ [132] } & Composition: & & & & \multirow[t]{10}{*}{ Not evaluated ${ }^{\mathrm{b}}$} \\
\hline & & Peptide mapping & Same & Same & & \\
\hline & & Glycan quantification & Same & Same & & \\
\hline & & $\begin{array}{l}\text { Mass spectrometry, intact } \\
\text { mass }\end{array}$ & Heterogeneous & Heterogeneous & & \\
\hline & & DSC analysis & Similar & Similar & & \\
\hline & & Cation exchange, acid (\%) & 7.0 & 22.1 & & \\
\hline & & Cation exchange, main (\%) & 20.6 & 68.5 & & \\
\hline & & Cation exchange, basic (\%) & 72.4 & 9.4 & & \\
\hline & & $\begin{array}{l}\text { Hydrophobic interaction } \\
\text { (main isoform) }(\%)\end{array}$ & $<24.1$ & $<2.0$ & & \\
\hline & & $\begin{array}{l}\text { Multi-angle laser light } \\
\text { scattering }\end{array}$ & Similar & Similar & & \\
\hline
\end{tabular}


Table 6 continued

\begin{tabular}{|c|c|c|c|c|c|c|}
\hline Study type [patients $(n)$ ] & Reference & Outcome & Intended copy ${ }^{\mathrm{a}}$ & Rituximab $^{\mathrm{a}}$ & $\begin{array}{l}\text { Statistical } \\
\text { comparison }\end{array}$ & $\begin{array}{l}\text { Quality assessment } \\
\text { rating }\end{array}$ \\
\hline \multirow[t]{4}{*}{ Analytical } & \multirow[t]{4}{*}{ CA [134] } & Composition: & & & & Not evaluated ${ }^{\mathrm{b}}$ \\
\hline & & IdeS digestion & Similar & Similar & & \\
\hline & & $\begin{array}{l}\text { Peptide mapping (trypsin } \\
\text { and pepsin) }\end{array}$ & Similar & Similar & & \\
\hline & & Isotope & Similar & Similar & & \\
\hline \multirow[t]{5}{*}{ Analytical } & \multirow[t]{5}{*}{ CA [135] } & Composition: & & & & Not evaluated ${ }^{\mathrm{b}}$ \\
\hline & & SDS-PAGE & Similar & Similar & & \\
\hline & & $\mathrm{iCE}$ & NR & NR & Significant & \\
\hline & & $\mathrm{CE}$ & NR & NR & Significant & \\
\hline & & CEX-HPLC & NR & NR & Significant & \\
\hline \multicolumn{7}{|l|}{ Kikuzubam $^{\circledR}$} \\
\hline \multirow[t]{3}{*}{ Nonclinical (cell-based) } & \multirow[t]{3}{*}{ [132] } & $\begin{array}{l}\text { Functional assessment } \\
\text { (in vitro): }\end{array}$ & & & & Not evaluated ${ }^{\mathrm{b}}$ \\
\hline & & $\operatorname{ADCC}(\%)$ & $80-125$ & $80-125$ & NS & \\
\hline & & CDC (3 batches) $(\%)$ & $98 ; 102 ; 112$ & $\begin{array}{l}\mathrm{NR} / 81 ; 111 ; \\
\quad 108\end{array}$ & NS & \\
\hline \multirow{10}{*}{$\begin{array}{l}\text { Analytical/nonclinical (cell- } \\
\text { based) }\end{array}$} & \multirow[t]{10}{*}{ [132] } & Composition: & & & & Not evaluated $^{\mathrm{b}}$ \\
\hline & & Peptide mapping & Same & Same & & \\
\hline & & Glycan quantification & Same & Same & & \\
\hline & & $\begin{array}{l}\text { Mass spectrometry, intact } \\
\text { mass }\end{array}$ & Heterogeneous & Heterogeneous & & \\
\hline & & DSC analysis & Similar & Similar & & \\
\hline & & Cation exchange, acid (\%) & 37.8 & $22.1 / 7.0$ & & \\
\hline & & Cation exchange, main (\%) & 56.6 & $68.5 / 20.6$ & & \\
\hline & & Cation exchange, basic (\%) & 5.6 & $9.4 / 72.4$ & & \\
\hline & & $\begin{array}{l}\text { Hydrophobic interaction } \\
\text { (main isoform) }(\%)\end{array}$ & $<3.0$ & $<2.0 /<24.1$ & & \\
\hline & & $\begin{array}{l}\text { Multi-angle laser light } \\
\text { scattering }\end{array}$ & Similar & Similar & & \\
\hline
\end{tabular}

ACR20 American College of Rheumatology $20 \%$ improvement criteria, $A D A b$ anti-drug antibodies, $A D C C$ antibody-dependent cell-mediated cytotoxicity, $A E$ adverse event, $B L$ baseline, $C A$ conference abstract, $C D C$ complement-dependent cytotoxicity, $C E$ capillary electrophoresis, $C E X-H P L C$ cationexchange chromatography high-performance liquid chromatography, $C R P$ C-reactive protein, $D \& B$ Downs and Black (tool), $D A S$ Disease Activity Score, $D A S 28$ Disease Activity Score in 28 joints, DMARD disease-modifying antirheumatic drug, DSC differential scanning calorimetry, ESR erythrocyte sedimentation rate, $i C E$ imaged capillary electrophoresis isoelectric focusing, IdeS Immunoglobulin-degrading enzyme from Streptococcus pyogenes, $L D A$ low disease activity, $N A$ not available, $N R$ not reported, $P P S$ per protocol set, $R A$ rheumatoid arthritis, SDS-PAGE sodium dodecyl sulfate polyacrylamide gel electrophoresis

${ }^{a}$ Qualitative data for biosimilarity as stated by the corresponding study authors

${ }^{\mathrm{b}}$ Quality assessment not conducted due to the absence of validated tools specific for the study type, at the time of analysis

effectiveness ratio (ICER) was most sensitive to the acquisition cost of Yisaipu ${ }^{\circledR}$.

\subsubsection{Intended Copies of Rituximab}

Studies of rituximab intended copies are presented in Table 6.

Kikuzubam ${ }^{\circledR}$ (Probiomed) The biochemical characterization and in vitro biological activity of Kikuzubam ${ }^{\circledR}$ (and Reditux $^{\mathrm{TM}}$ ) versus rituximab have been assessed in a preclinical study [132]. Study findings provided evidence of similar potency to rituximab. As previously mentioned, an observational study investigating Kikuzubam ${ }^{\circledR}$ (or Infini$\operatorname{tam}^{\circledR} / \mathrm{Yisaipu}^{\circledR}$ ) in patients with rheumatic diseases reported that a large proportion of patients experienced AEs [125].

Reditux $^{T M}$ (Dr Reddy's Laboratories) Reditux ${ }^{\mathrm{TM}}$ has been evaluated in two independent nonclinical studies, wherein the drug was reported to exhibit similar biological effects in vitro to rituximab (in cell-based assays) [132, 133]. Two nonclinical studies examined structural attributes of Reditux $^{\mathrm{TM}}$ and reported heterogeneity between it and rituximab with respect to theoretical mass and secondary/tertiary structure [132, 135, 134]. Three observational trials were identified that assessed clinical outcomes for Reditux $^{\mathrm{TM}}[130,128,129,131]$. Although none of these studies provided a comparator, there is some evidence supporting the efficacy and safety of Reditux ${ }^{\mathrm{TM}}$ 
in the treatment of chronic inflammatory disease and, in particular, for treatment of RA.

\subsection{Quality Assessment of the Studies}

The quality of all included randomized and non-randomized studies were assessed using validated instruments.

\subsubsection{National Institute for Health and Care Excellence (NICE) Single Technology Appraisal (STA) Manufacturer's Template}

Seven RCTs were assessed using the NICE STA manufacturer's template [42, 44, 49, 33, 101, 69, 59] (Tables 1, 2, 3, ESM Fig. S3a); all were considered excellent quality overall. The RCTs provided information to assess the randomization process, subject withdrawals, outcome selection, reporting bias, and statistical analyses, which were adequately reported in all studies. The bias of allocation concealment process was unclear in three studies (Jani et al. [33], Yi et al. [44], and Yoo et al. [69]). The blinding process was unclear in one study (Yoo et al. [69]) and assessed as high risk for unblinding in one study ( $\mathrm{Gu}$ et al. [42]).

\subsubsection{Jadad Scoring Tool}

Seven RCTs were evaluated using the Jadad scoring tool (Tables 1, 2, 3, ESM Fig. S3b). Choe et al. [101] and Emery et al. [49] scored high for reporting on the five scored regions of randomization, blinding, methodology, withdrawals, and dropouts (total score 5 points). Gu et al. [42] reported an open-label study and, therefore, scored the maximum possible 3 points [42]; Jani et al. [33] and Park et al. [59] both failed to report on the method of blinding, and therefore scored 4 points each; Yi et al. [44] and Yoo et al. [69] failed to report on both the randomization and blinding methods, thereby scoring 3 points each.

\subsubsection{Downs and Black/Modified Downs and Black Instrument}

Four observational studies with full-study reporting were assessed using the Downs and Black scoring tool (Tables 3 and 6, ESM Fig. S4) [80, 76, 78, 131]; all were considered fair quality. The restrictive word count in abstracts from conference proceedings generally provide limited information on study methodologies and outcomes. For this reason, the Downs and Black instrument was adapted to assess the quality of the 22 identified abstracts for additional original studies (Tables 1, 2, 3, 4, 5, 6, ESM Fig. S5) $[26,41,47,48,98,63,46,419,122$, 103, 81, 74, 79, 82, 124, 125, 126, 130, 128, 104, 50, 34].
The average score for the abstract publications was 7.8 out of a possible 12 points. The total score was fair quality (3-4) for four studies [81, 74, 125, 130], good quality (5-8) for seven studies [48, 122, 79, 82, 126, 128, 104], and excellent quality (9-12) for 11 studies [26, 41, 47, 98, 63, 45, 119, 103, 124, 50, 34]. The majority of the studies published as conference abstracts were of good or excellent quality $(81.8 \%)$.

\subsubsection{Drummond's Checklist}

Three pharmacoeconomic studies were assessed using Drummond's checklist for assessing economic evaluations (Tables 3 and 5, ESM Fig. S6) [84, 87, 127]; the studies were of good quality. Of a maximum 36 points, Brodszky et al. [84] scored 23 points, Jha et al. [87] scored 23 points, and $\mathrm{Wu}$ et al. [127] scored 29 points.

\subsubsection{SYRCLE's Risk of Bias Tool}

Three animal studies were assessed using SYRCLE's risk of bias tool (Tables 2, 4, ESM Fig. S7) [52, 115, 108]. The three studies were of moderate quality.

\subsection{Weight and Breadth of Evidence for Biosimilarity}

The final determination of biosimilarity by regulatory authorities (e.g., the FDA [136]) is based on the 'totality of evidence' approach and the degree of residual uncertainty concerning biosimilarity between the proposed biosimilar and the innovator drug. The authorities base their decision on data from the molecular and functional characterization, any nonclinical data obtained, and the safety, pharmacokinetic, immunogenicity, and efficacy clinical trial data provided by the manufacturer. However, given that regulatory submissions are not publically available and the full dataset therein cannot be systematically reviewed, the authors have instead based all analysis in this literature review on information from the peer-reviewed literature.

All comparative publications uncovered in this literature review were carefully assessed to analyze the reported extent of similarity between the originator product and the biosimilar. Biosimilarity (based on combined evidence from all related published studies) for each type of study was graded as comparable, highly similar, similar, or nonsimilar, which was directly inferred from investigator conclusions (Table 7). The available comparative data from full-text publications of randomized clinical trials are currently limited. However, based on original investigator conclusions from the literature at the time the search was undertaken, the current data from clinical, nonclinical, and observational/post-marketing studies for proposed 
Table 7 Summary of evidence for the degree of similarity between biosimilars/intended copies and originators by study type

\begin{tabular}{|c|c|c|c|c|c|c|}
\hline $\begin{array}{l}\text { Biologic } \\
\text { originator }\end{array}$ & $\begin{array}{l}\text { Biosimilar/intended } \\
\text { copy [name(s); } \\
\text { (company)] }\end{array}$ & $\begin{array}{l}\text { Analytical } \\
\text { studies }\end{array}$ & $\begin{array}{l}\text { Nonclinical } \\
\text { studies }\end{array}$ & $\begin{array}{l}\text { PK/safety studies } \\
\text { or preliminary } \\
\text { safety/efficacy } \\
\text { studies }\end{array}$ & $\begin{array}{l}\text { Comparative } \\
\text { safety/efficacy } \\
\text { studies }\end{array}$ & $\begin{array}{l}\text { Post- } \\
\text { marketing/ } \\
\text { observational } \\
\text { studies }\end{array}$ \\
\hline \multirow[t]{5}{*}{ Adalimumab } & ABP 501 (Amgen, USA) & $\boldsymbol{V}$ or $\boldsymbol{V} \boldsymbol{V}$ & $\checkmark v$ & $\checkmark v$ & NA & NA \\
\hline & GP2017 (Sandoz, Switzerland) & NA & $\checkmark v$ & NA & NA & NA \\
\hline & PF-06410293 (Pfizer, USA) & $\checkmark \checkmark$ & $\checkmark \checkmark$ & NA & NA & NA \\
\hline & $\begin{array}{l}\text { SB5 (Samsung Bioepis, South } \\
\text { Korea) }\end{array}$ & NA & NA & $\checkmark v$ & NA & NA \\
\hline & $\begin{array}{l}\text { ZRC-3197 } \text { (Exemptia }^{\mathrm{TM}} \text {; Cadila } \\
\text { Healthcare, India) }\end{array}$ & NA & NA & NA & $\checkmark v$ & NA \\
\hline \multirow[t]{6}{*}{ Etanercept } & $\begin{array}{l}\text { AVG01 (Avent }{ }^{\mathrm{TM}} \text {; Avesthagen, } \\
\text { India) }\end{array}$ & $\checkmark \checkmark$ & $\checkmark \boldsymbol{V}$ & NA & NA & NA \\
\hline & $\begin{array}{l}\text { ENIA11 }\left(\text { TuNEX }{ }^{\circledR} ; \text { Mycenax }\right. \\
\text { Biotech/TSH Biopharm Corp., } \\
\text { Taiwan) }\end{array}$ & NA & NA & $\checkmark v$ & NA & NA \\
\hline & GP2015 (Sandoz, Switzerland) & NA & $\checkmark v$ & NA & NA & NA \\
\hline & $\begin{array}{l}\text { HD203 (Davictrel }{ }^{\mathrm{TM}} ; \text { Hanwha } \\
\text { Chemical, South Korea/Merck, } \\
\text { USA) }\end{array}$ & NA & NA & $\checkmark v$ & $\checkmark v$ & NA \\
\hline & $\begin{array}{l}\text { LBEC0101 (LG Life Sciences, } \\
\text { South Korea) }\end{array}$ & NA & NA & $\checkmark v$ & NA & NA \\
\hline & $\begin{array}{l}\text { SB4 (Benepali }{ }^{\circledR} \text {; Samsung } \\
\text { Bioepis, South Korea) }\end{array}$ & NA & NA & $\checkmark v$ & $\checkmark \checkmark$ & NA \\
\hline \multirow[t]{4}{*}{ Infliximab } & $\begin{array}{c}\text { BOW015 (Infimab }{ }^{\circledR} \text {; Ranbaxy } \\
\text { Laboratories, India/Epirus } \\
\text { Biopharmaceuticals, USA) }\end{array}$ & NA & NA & $\checkmark v$ & $\checkmark v$ & NA \\
\hline & $\begin{array}{l}\text { CT-P13 }\left(\text { Remsima }{ }^{\circledR} ; \text { Inflectra }{ }^{\circledR} ;\right. \\
\text { Celltrion, South Korea/Hospira, } \\
\text { USA) }\end{array}$ & $\boldsymbol{V} \boldsymbol{V}$ or $\boldsymbol{V} \boldsymbol{V}$ & $\checkmark v$ & $\checkmark \checkmark$ & $\checkmark \checkmark$ & a \\
\hline & PF-06438179 (Pfizer, USA) & $\boldsymbol{V} \sim$ or $\boldsymbol{V} \boldsymbol{V}$ & $\checkmark v$ & $\checkmark \boldsymbol{V}$ & NA & NA \\
\hline & $\begin{array}{l}\text { SB2 (Flixabi }{ }^{\circledR} \text {; Samsung Bioepis, } \\
\text { South Korea) }\end{array}$ & NA & NA & $\checkmark v$ & $\checkmark \checkmark$ & NA \\
\hline \multirow[t]{4}{*}{ Rituximab } & $\begin{array}{l}\text { CT-P10 (Celltrion, South Korea/ } \\
\text { Hospira, USA) }\end{array}$ & NA & NA & $\checkmark v$ & NA & NA \\
\hline & GP2013 (Sandoz, Switzerland) & $\checkmark v \checkmark$ & $\checkmark$ & NA & NA & NA \\
\hline & PF-05280586 (Pfizer, USA) & $\boldsymbol{V} \boldsymbol{v}$ or $\boldsymbol{V} \boldsymbol{V}$ & $\checkmark v$ & $\checkmark v$ & NA & NA \\
\hline & $\begin{array}{l}\text { RTXM83 (mAbxience, } \\
\text { Switzerland) }\end{array}$ & $\checkmark$ & $\checkmark$ & NA & NA & NA \\
\hline \multirow[t]{4}{*}{ Intended copies } & Infinitam $^{\circledR}$ (Probiomed, Mexico) & NA & NA & $\checkmark v$ & NA & a \\
\hline & $\begin{array}{c}\text { Yisaipu }^{\circledR} \text { (Etanar }{ }^{\circledR} \text {; Shanghai CP } \\
\text { Guojian Pharmaceutical, China) }\end{array}$ & NA & NA & NA & NA & a \\
\hline & $\begin{array}{l}\text { Reditux }^{\mathrm{TM}} \text { (Dr Reddy's } \\
\text { Laboratories, India) }\end{array}$ & $\boldsymbol{*}$ or $\boldsymbol{V} \boldsymbol{V}$ & $\checkmark$ & NA & NA & a \\
\hline & $\begin{array}{l}\text { Kikuzubam }^{\circledR} \text { (Probiomed, } \\
\text { Mexico) }\end{array}$ & $\boldsymbol{*}$ or $\boldsymbol{V} \cup \boldsymbol{V}$ & $\checkmark v$ & NA & NA & $\mathrm{a}$ \\
\hline
\end{tabular}

$N A$ not applicable, evidence from published studies not available, $P K$ pharmacokinetic, $S A E$ serious adverse events, $\boldsymbol{V} \boldsymbol{V}$ identical (based on combined evidence from all related published studies), $\boldsymbol{V} \boldsymbol{V}$ highly similar (based on combined evidence from all related published studies), $\boldsymbol{V}$ similar (based on combined evidence from all related published studies), $\boldsymbol{x}$ non-similar (based on combined evidence from all related published studies)

${ }^{a}$ Not possible to draw conclusions from published studies, due to the lack of direct comparative data with the originator

biosimilars indicate moderate to high levels of biosimilarity to the comparator products (Table 7).

In order to summarize findings, the total number of studied variables and total reported patient numbers were extracted from the identified analytical/nonclinical and clinical studies, respectively. These parameters were then mapped (Fig. 2a, b) against the 'degree of similarity' (as observed by the study investigator) in an effort to depict the 

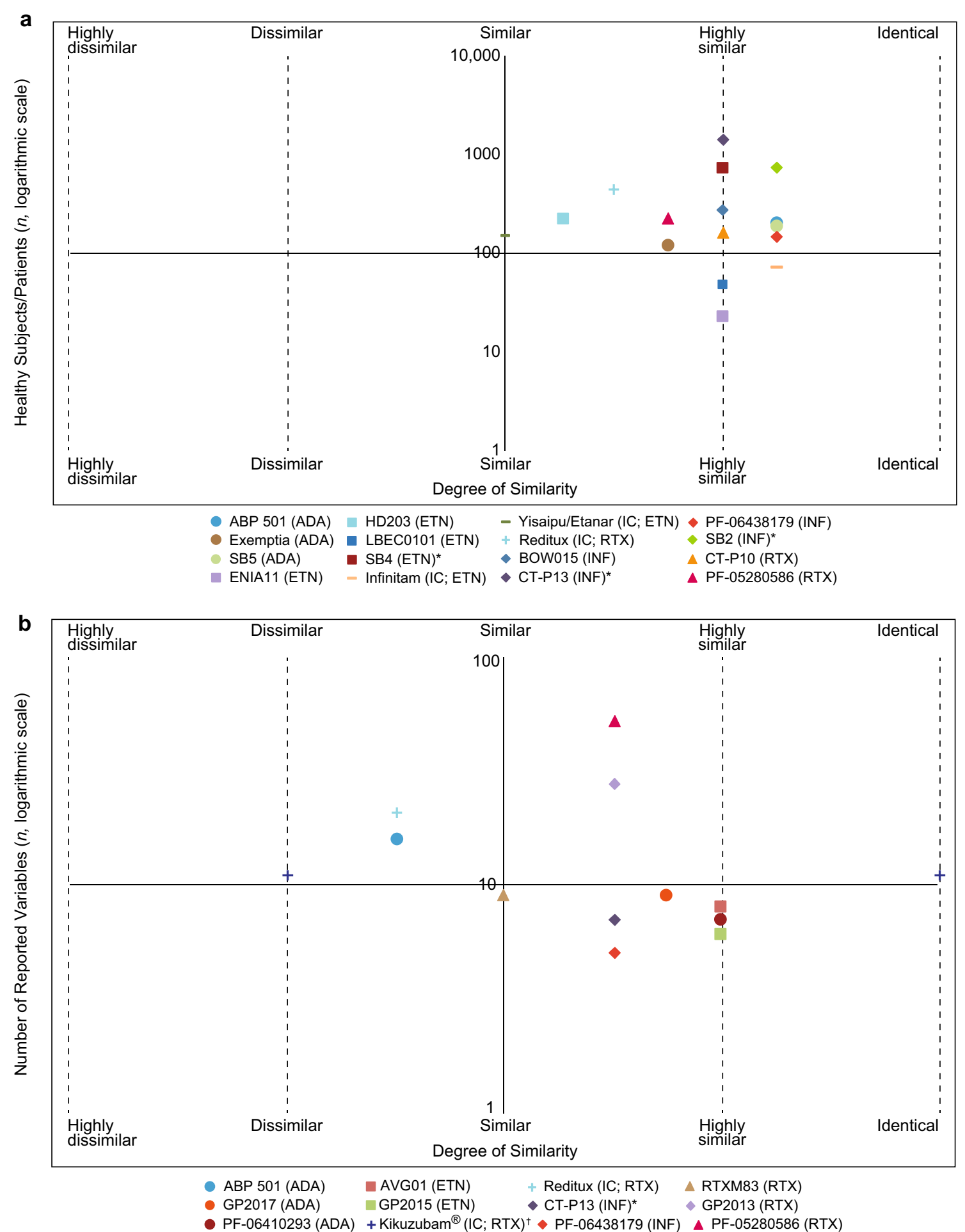

Fig. 2 Biosimilarity and a total treated patients for named biosimilars and intended copies in published clinical trials and $\mathbf{b}$ breadth of data for named biosimilars and intended copies in published analytical and nonclinical studies. 'Degree of similarity' for biosimilars and intended copies is inferred from the totality of evidence provided from all available published studies (up to 3 September 2015), and is based on the original conclusions made by the study investigators. The scale of reference used by each investigator was not accounted for, as it was not uniformly reported. * refers to agents that have already met the European Medicines Agency and/or US Food and Drug Administration requirements and have been approved as biosimilars. $\dagger$ based on different author interpretations of study data, intended copy Kikuzubam $^{\circledR}$ purportedly exhibits some highly dissimilar and some identical physicochemical characteristics compared with the originator. ADA adalimumab, ETN etanercept, IC intended copy, $I N F$ infliximab, $R T X$ rituximab 
overall quantity of available evidence for each agent. Most biosimilars have published clinical data on fewer than 100 human subjects, whereas studies of CT-P13 have involved more than 1000 subjects (Fig. 2a). Studies of 13 biosimilars identified included 4522 patients or healthy subjects. Approved biosimilars CT-P13 (infliximab biosimilar Remsima ${ }^{\circledR} /$ Inflectra ${ }^{\circledR}$ ), SB2 (infliximab biosimilar, Flix$\mathrm{abi}^{\circledR}$ ), and SB4 (etanercept biosimilar, Benepali ${ }^{\circledR}$ ) reported the largest combined study populations from pharmacokinetic/safety, comparative efficacy/safety, and observational studies (1405, 743, and 734 patients, respectively). Four intended copies were identified in studies including a total of 1430 patients.

When considering the breadth of data available for preclinical studies (based on the number of variables studied in structural, functional, and nonclinical studies) for named biosimilars, there is an inconsistent amount of reported information available across studies (Fig. 2b). PF05280586 (rituximab) and GP2013 (rituximab) were found to have a larger number of investigated variables for analytical and nonclinical biosimilarity. Of note, Flores-Ortiz et al. [132] reported that the mass spectrometry and cation exchange findings were heterogeneous for the intended copy Kikuzubam ${ }^{\circledR}$ in comparison to its rituximab originator, while other variables (differential scanning calorimetry analysis, peptide mapping, glycan quantification, etc.) were reported to be the same. Thus, the positioning on the $x$ axis (Fig. 2b) was determined to be both dissimilar and identical across selected variables.

Although the original study investigators concluded that the majority of agents exhibited biosimilarity to their originator, it is worth noting that comparative data were not provided for all attributes studied.

\subsection{Non-Empirical Publications}

A summary of information from the non-empirical publications is shown in ESM Table S5. Within the 34 identified empirical publications, 16 were therapy area overviews (in $\mathrm{RA}, \mathrm{AS}, \mathrm{UC} / \mathrm{CD}$, and dermatology) and 11 articles discussed regulatory policy. Other topics included biosimilar development, national guidelines, safety/pharmacovigilance, and substitution/interchangeability. One theme that was discussed in many of the articles (at least 17 of the 34 publications) was the extrapolation of clinical data from clinical trials of biosimilars between different indications. In many of the 17 publications, the extrapolation of clinical trial data of the biosimilar infliximab CT-P13 (Remsima ${ }^{\circledR} /$ Inflectra ${ }^{\circledR}$ ) from RA patients to patients with UC/CD was discussed in detail due to the fact that CT-P13 was originally approved by the EMA, but not the Canadian authorities, for the treatment of UC/CD. Whilst the EMA considered that "high similarity" in preclinical studies together with clinical data from two trials in AS and RA warrant the "extrapolation" for CD and UC, Canadian authorities did not initially accept extrapolation, based on differences in glycosylation (fucosylation) in irritable bowel syndrome. However, CT-P13 has now been approved by Health Canada for additional indications, including $\mathrm{CD}$ and $\mathrm{UC}$.

\subsection{Additional Planned and Ongoing Trials of Adalimumab, Etanercept, Infliximab, and Rituximab Biosimilars}

The ClinicalTrials.gov registry was consulted to identify planned or ongoing trials not yet reported in the published literature. Trials were listed for biosimilars for adalimumab in a total of 14 studies (ESM Table S6). The clinical trials search also identified biosimilars of adalimumab not yet appearing in the published literature. Biosimilars produced by Coherus Biosciences, Inc. (USA), LG Life Sciences (South Korea), Boehringer Ingelheim (Germany), and Biocad (Russia) were identified for adalimumab (CHS1420, LBAL, BI 695501, and BCD-057, respectively).

A total of nine studies were listed in the ClinicalTrials.gov database for named biosimilars of etanercept (ESM Table S6). Trials were also identified for a newly identified biosimilar, CHS-0214 (Coherus Biosciences, Inc.; two studies co-sponsored with Daiichi Sankyo Co. Ltd. and one with Baxalta US Inc.).

A total of three clinical trials were retrieved from the search for named biosimilars of infliximab in ClinicalTrials.gov (ESM Table S6). An additional biosimilar, BCD055 (Biocad), was identified from this search, which was not identified in the published literature search.

Seven trials were identified in the ClinicalTrials.gov search for named biosimilars of rituximab for the treatment of RA only (ESM Table S6). One biosimilar (BI 695500) that had not been reported in the published literature was identified from this search.

Although the majority of patient studies listed for development candidates of adalimumab, etanercept, and infliximab were in RA (for ABP 501 [adalimumab], BI 695501 [adalimumab], PF-06410293 [adalimumab], SB5 [adalimumab], CHS-0214 [etanercept], ENIA11 [etanercept], LCEC0101 [etanercept], and PF-06438179 [infliximab]), several complete or ongoing studies were also identified for psoriasis or plaque psoriasis for ABP 501, CHS-1420 (adalimumab), GP2017 (adalimumab), CHS-0214, and GP2015 (etanercept). In addition, for infliximab biosimilars BCD-055 and CT-P13, studies were recruiting in $\mathrm{AS}$ and $\mathrm{CD}$, respectively, at the time of analysis. 


\subsection{Key Journals and Congresses}

A total of 98 journals publishing relevant material on biosimilars for chronic inflammatory disease were identified during this review (ESM Table S7). Annals of the Rheumatic Diseases published the greatest number of articles on this topic during the search period (ten articles since 2013). Prior to 2011, no journal articles relevant to the topic of MAb or fusion protein biosimilars were published that were relevant to chronic inflammatory diseases and there has been a steady rise since then (two, six, ten, and 14 articles in 2011, 2012, 2013, and 2014, respectively). In the last complete year of the analysis (2014), the European Journal of Health Economics published the greatest number of articles related to biosimilars in chronic inflammatory disease (four articles), followed by Annals of the Rheumatic Diseases (three articles), and the Journal of Crohn's and Colitis (three articles).

A total of 28 congresses were identified that presented abstracts of named biosimilars relevant to chronic inflammatory diseases between January 2010 and August 2015 (ESM Table S8). No congress abstracts were identified in the years preceding 2010. A total of 54 abstracts were published in the year 2014 for these congresses. Of these, the largest number of abstracts were published for dissemination at European League Against Rheumatism (EULAR) (17 abstracts), ACR (13 abstracts), and International Society For Pharmacoeconomics and Outcomes Research (ISPOR) (ten abstracts) congress meetings. The sharp increase in the number of abstract publications from 2013 to 2014 at these congresses (30 to 54 abstracts) highlights the high level of interest in biosimilars for the treatment of chronic inflammatory diseases among clinical, regulatory, and payer stakeholders. The search also captured abstracts published between January and August 2015. A total of 22 abstracts were presented at EULAR and 11 at European Crohn's and Colitis Organisation (ECCO) congress meetings.

\section{Discussion}

Guidelines require that biosimilars should exhibit close similarity to the originator with respect to structure (primary and higher-order) and many other molecular characteristics, display similar levels of biological potency in vitro or in vivo, and should meet the required thresholds for bioequivalence in safety and pharmacokinetic studies before entering a comparative trial(s) in patients to satisfy efficacy requirements [2]. Robust evidence of similarity provided from analytical, pharmacokinetic, and nonclinical studies is thus equally as important as clinical evidence to demonstrate the safety and efficacy of a biosimilar, and to meet regulatory standards and requirements set by the EMA and FDA for approval $[137,138]$. While these data are generated as evidence for approval, ensuring the data are made available in the public domain is also important for promoting education and awareness and encouraging acceptance of biosimilars among physicians and other healthcare professionals.

At the time of data analysis, the totality of evidence for candidate biosimilars with comparative studies in chronic inflammatory disease suggested that the majority of biosimilars demonstrated a moderate to high degree of similarity to their originator. In contrast, there was limited information in the public domain regarding the similarity of intended copies with their originator products.

This SLR revealed several biosimilar molecules that met the required standards. CT-P13 (Remsima ${ }^{\circledR} /$ Inflectra ${ }^{\circledR}$ ) is the only commercially available infliximab biosimilar to date that has been launched across 12 European countries and Canada, and approved in the USA. The combined evidence gathered from analytical, nonclinical, and clinical studies (identified in this review of publications to 3 September 2015) supported the premise that CT-P13 is safe and effective $[63,91,90,66,121,67,72,69,71$, $64,139,92,73,70,68]$. A comparative safety/efficacy study is underway in CD and CT-P13 is also being evaluated in observational studies for multiple other chronic inflammatory conditions (including ankylosing spondyloarthritis, PsA, UC, CD, and chronic psoriasis) [63, 59, $58,61,123,62,80,81,76,77,78,74,79,75,82,64$, $68,65,60]$. PF-06438179 is another infliximab biosimilar that has exhibited moderate to high structural and functional similarity during analytical and preclinical investigations [94, 95, 93, 98, 99, 97, 96]. At the time of this review, a comparative safety/efficacy trial comparing the efficacy and immunogenicity of PF-06538179 versus infliximab was recruiting patients with RA. Adalimumab biosimilars GP2017 and PF-06410293 both showed similarity to adalimumab in in vitro assays and preclinical studies [37, 36, 35, 38, 39]. At the time of analysis, progression of PF-06410293 into three pharmacokinetic/safety studies and one comparative safety/efficacy trial had been listed on ClinicalTrials.gov and comparative safety/efficacy trials for GP2017 were underway in the treatment of plaque psoriasis. For GP2015, an etanercept biosimilar, published nonclinical (in vitro and in vivo) data revealed close functional similarity compared with its originator [53]. Finally, rituximab biosimilars GP2013 and PF05280586 both showed adequate degrees of similarity to rituximab during preclinical phases of development $[117,115,116]$. Some pharmacokinetic/safety trials and preliminary safety/efficacy trials for RA have also been reported [114, 109, 111, 113, 108, 112, 104, 106, 107, 105]. 
For the prescribing physician and key decision-makers, it is important to understand whether the published data on biosimilarity are of sufficient quality to influence prescribing decisions. Therefore, as well as investigating the weight of available data, this review also used validated instruments, such as the NICE STA assessment, Jadad scoring tool, Downs and Black checklist, Drummond scoring system for economic studies, and SYRCLE's risk of bias tool for animal studies to assess the quality of studies. The results were reassuring; of the seven RCTs assessed, all were considered to be of excellent quality by the NICE STA assessment, and all scored between 3 and 5 points using the Jadad scoring tool.

Taken together, the weight and breadth of evidence of comparative studies suggest that biosimilars will soon become a mainstay of treatment in many different chronic inflammatory diseases. The non-empirical publication analysis in this report suggests that the reduced cost of biosimilars and the increased number of available therapeutic options may make access to biologic therapy available to a broader subset of patients. This is particularly important for chronic diseases such as RA, AS, UC, and $\mathrm{CD}$ where patients need treatment with costly biologic therapy for long periods of time [6]. Indeed, it has often been shown in these diseases that early aggressive therapy has long-term benefits both for the prevention of joint deterioration (in the case of RA) [140] and quality of life [141, 142]. The availability of relatively low-cost biosimilars may eventually alter the treatment paradigm so that patients are treated for less time with conventional diseasemodifying antirheumatic drugs alone, and biologic therapy is initiated earlier in the disease course [143].

Although the number and quality of published studies providing evidence of structural and functional similarity at the preclinical phase may not be sufficient to predict behaviors or performance in humans, these studies form the basis for demonstration of similarity and represent a crucial step in the development program. At the time of analysis, BOW015 (Infimab ${ }^{\circledR}$; infliximab; Ranbaxy Laboratories, India/Epirus Biopharmaceuticals), CT-P10 (infliximab; Celltrion), SB2 (Flixabi ${ }^{\circledR}$; infliximab; Samsung Bioepis); SB5 (adalimumab, Samsung Bioepis), and ZRC-3197 (Exemptia $^{\mathrm{TM}}$; adalimumab; Cadila Healthcare) had all entered into clinical stages of development (comparative safety/efficacy in RA) without published data or evidence to suggest that they had similar structural or functional resemblance to their originators. This was also true for the majority of etanercept biosimilars (HD203 [Davictrel ${ }^{\mathrm{TM}}$; Hanwha Chemical], LBEC0101 [LG Life Sciences], ENIA11 [TuNEX ${ }^{\circledR}$; Biotech/TSH Biopharm Corp., Taiwan], and SB4 [Benepali ${ }^{\circledR}$; Samsung Bioepis]), which had all reported comparative safety/efficacy trials in RA with a lack of published analytical or nonclinical data. Although publication of similarity information is not a regulatory requirement for the approval of biosimilars, a lack of information in the public domain will impact on education and awareness and therefore the ability of clinicians to make informed prescribing decisions.

This review also provides information on marketed intended copies (copies of originator biologics that have not undergone rigorous comparative evaluations). Several intended copies are commercially available with very little evidence of biosimilarity. For example, intended copy Kikuzubam $^{\circledR}$ (Probiomed) gained approval in Mexico without published comparative safety/efficacy trials and prior to COFEPRIS releasing official regulatory guidance on 'biocomparables' [17]. In 2012, Kikuzubam ${ }^{\circledR}$ was withdrawn by the regulatory authority due to documented anaphylactic reactions and a lack of clinical data [144]. Intended copy Reditux ${ }^{\mathrm{TM}}$ (Dr Reddy's Laboratories) was also commercialized in Latin American countries, India, and Iran without published data to indicate structural or functional similarity or clinical effectiveness as compared with rituximab [145]. Although several observational studies have been reported for Reditux ${ }^{\mathrm{TM}}$, no studies for chronic inflammatory conditions included rituximab as a comparator at the time of this review [132, 133, 135, 134, 130, 128, 129, 131]. Intended copy Yisaipu $^{\circledR}$ (etanercept; Shanghai CP Guojian Pharmaceutical Co.) is a fusion protein that is already marketed in China. However, as is evident from this review, no published data are available for comparison with etanercept to determine its biosimilarity. In Colombia, Yisaipu ${ }^{\circledR}$ is marketed under the brand name Etanar ${ }^{\circledR}$. At the time of this review, only one limited clinical trial (not an equivalence study comparing Yisaipu $^{\circledR}$ with etanercept) had been reported [48, 126, 127]. Finally, at the time that this analysis was completed, there was only one study that assessed the efficacy and safety of intended copy Infini$\operatorname{tam}^{\circledR}$, which is currently marketed in Mexico [124]. Suffice to say, significant data gaps remain for intended copies, reinforcing the need to maintain a clear differentiation between these molecules and true biosimilars.

Non-empirical data analysis suggests that some countries are only just beginning to establish formal regulatory guidelines for the approval of biosimilars to cover requirements for preclinical, clinical, or other analyses that should be used to demonstrate the safety, quality, and effectiveness of a biosimilar. This includes studies required for immunogenicity and AEs, as well as demonstration of similar modes of action or pharmacodynamic properties to that of the originator. Other countries have yet to implement any guidelines. The extrapolation of biosimilarity information across indications was also an issue that was mentioned frequently in the non-empirical publications identified in this report [144, 146, 147, 
148, 149, 150, 151, 152, 153, $154,155,156,157,158,159,160]$. A common theme of discussion was the fact that RA (where almost all molecules were first investigated) may not be a sensitive clinical model to detect potential differences between biosimilars and originator products that may be observed in UC/CD. It was suggested that the immunogenicity potential of biosimilars is fairly low in RA and even further suppressed by the concomitant use of methotrexate, and that regulatory agencies should grant extrapolated indications to biosimilars based on appropriate scientific justification only after biosimilarity is confirmed [152, 154].

This systematic review aims to provide a comprehensive summary of literature relevant to $\mathrm{mAb}$ and fusion protein biosimilars for the treatment of chronic inflammatory diseases. However, there are some inherent weaknesses and limitations to this method of data analysis. As with any systematic review, the search retrieved records citing a particular term (either a mAb or fusion protein biosimilar). Due to a lack of consensus on the naming of biosimilars and intended copies, no consistent nomenclature could be relied upon to identify and correctly classify every possible molecule of interest. Molecules may, for example, have been incorrectly termed biosimilars in the literature without rigorous data to support biosimilarity to the originator product. The authors' determination of biosimilarity for each molecule was limited to using only the specific terms (e.g., "similar" or "highly similar") reported by the investigators. Consequently, the analysis is based purely on the scale of reference used by the original investigators.

In addition, only data from studies disclosing names of biosimilars or intended copies (i.e., utilizing a unique identifier) and from journals or abstracts published in English were extracted. Clinical trial information was taken from the ClinicalTrials.gov database; no other clinical trial registries were consulted in this analysis. It is therefore possible that information on some regional trials may not have been captured.

One of the original aims of our research was to identify gaps in the published literature. With this in mind, the results presented contain only the outcomes data and statistical comparisons available from the published abstracts or full-text articles retrieved from the search. Thus, the information collated may not be representative of the full extent of data available for each study, only that which has been published. It should be noted that the search strategy was designed to capture only articles published by MEDLINE $^{\circledR}$ - or EMBASE ${ }^{\circledR}$-indexed journals. Therefore, studies published by non-indexed journals were not included. A full Internet search of all online content was not included in the methodology.

It should also be noted that proceedings from only 17 conferences were searched, and thus additional data may be available from other conference proceedings not considered in this analysis. Furthermore, at the time of analysis, only limited outcomes data were available from published conference abstracts, with no full-text publications available at the time of the review.

Lastly, the authors also acknowledge that the delay between regulatory submission and publication of studies may partially explain why there is limited information available for those biosimilars that are newly approved.

A number of biosimilars have newly published data and new molecules (which were not included in this review) may now also be in development for the treatment of chronic inflammatory diseases. For example, at the time of writing, comparative safety/efficacy trials were listed in the ClinicalTrials.gov registry for M923 (adalimumab; Baxalta) for the treatment of chronic plaque-type psoriasis and moderate to severe RA, and for MYL-1401A (adalimumab; Mylan) in patients with psoriasis and PsA. In addition, other studies of biosimilars included in the review have since been listed in the ClinicalTrials.gov registry. For the infliximab biosimilar CT-P13 (Remsima ${ }^{\circledR} /$ Inflectra ${ }^{\circledR}$ ), two observational studies (one in RA and one in AS) and one post-marketing study (in RA patients [PERSIST]) have been listed. BCD-055 (infliximab biosimilar) has comparative safety/efficacy trials planned in AS and RA. Three new studies for adalimumab biosimilars have also been added. A multicenter clinical study to evaluate the usability and safety of a pre-filled pen and pre-filled syringe in RA is reportedly complete (SB5), GP2017 has a comparative safety/efficacy RA trial listed (ADMYRA), and there is a comparative safety/efficacy trial planned for BCD-057 in the treatment of plaque psoriasis (CALYPSO). For etanercept biosimilars, GP2015 is being studied (EQUIRA) and LBEC0101 is under evaluation for the treatment of RA. The rituximab biosimilar BCD-020 is being investigated with methotrexate as first-line biologic therapy for patients with active RA (ALTERRA). The number of new biosimilars and clinical trials identified illustrates the speed at which this field of medicine is progressing. This is a highly competitive market, as illustrated by Epirus Biopharmaceuticals suspending development of the biosimilar BOW015 (Infimab ${ }^{\circledR}$; infliximab) in May 2016, citing the evolving biosimilar competitive and business landscape as the primary reason [161].

As discussed here, there is a need to recognize and maintain a clear distinction between biosimilars and intended copies. However, since it is not always apparent from publicly available sources what manufacturers' intentions are, the term 'proposed biosimilars' is employed in this analysis as a blanket term for all development candidates pending final determination of their status as biosimilars. 
There are examples of molecules that are currently marketed as biosimilars in countries outside of the EU or USA: HD203 (Davictrel $^{\mathrm{TM}}$; Hanwha Chemical) was approved by the Korean MFDS in 2014 [162] and BOW015 (Infimab ${ }^{\circledR}$; infliximab; development now suspended) and ZRC-3197 (Exemptia ${ }^{\mathrm{TM}}$; adalimumab) were also approved in India [145]. As noted by Castañeda-Hernández et al. [145], it is not yet known whether other countries will approve these products as biosimilars based on the data currently available.

\section{Conclusions}

This SLR sought to collate and synthesize publicly available information on biosimilars on the market and in development for chronic inflammatory disease to support healthcare decision-making. As anticipated, at the time of this review, all approved biosimilars had published data providing evidence of biosimilarity from RCT studies. At the analysis cut-off, only CT-P13 had additional published evidence from analytical and nonclinical studies.

A number of proposed biosimilars are also amassing a significant body of evidence from studies across development stages. The majority of agents were found to exhibit a high degree of similarity to their originators, based on investigator conclusions. However, the authors found that significant gaps in the evidence base for some pipeline products remain, and this is also true for all of the intended copies identified. As shown in this review, only one intended copy had published RCT data and this was from a preliminary safety/efficacy study. The implications of these intended copies being marketed without a transparent evidence base are multi-fold and the authors advise caution in the use of these molecules. Apart from the obvious safety issues, this lack of transparency may have a broad impact on adoption of biosimilars in general and cause confusion among healthcare stakeholders. While the authors acknowledge that the existence of data not yet published may potentially address these gaps, if it is not in the public domain it will do little to alleviate these concerns.

RA is the first market in which multiple biosimilars have been launched and, undoubtedly, experience in this market will influence how the broader sector evolves. Therefore, the ongoing dissemination of data by all manufacturers is imperative to instill confidence and support adoption of biosimilars in healthcare practice.

Acknowledgments Medical writing support was provided by Robyn Fowler, PhD, of Engage Scientific Solutions, and was funded by Pfizer Inc. Carole Jones of Envision Pharma Group was involved with the development of the SLR, which was funded by Pfizer Inc.

\section{Compliance with Ethical Standards}

Author Contributions All authors were involved in drafting the article and revising it critically for important intellectual content. All authors read and approved the final manuscript submitted for publication.

Conflict of interest IJ, DP and KLS are full-time employees and shareholders of Pfizer. LI was a full-time employee of Pfizer Inc. at the time the study was conducted. SL is a full-time employee of Envision Pharma Group, who were paid consultants to Pfizer in connection with the development of the SLR report that forms the basis of this manuscript. He was not compensated for his role in the development of this manuscript.

Funding The SLR to support this manuscript was sponsored by Pfizer Inc.

Open Access This article is distributed under the terms of the Creative Commons Attribution-NonCommercial 4.0 International License (http://creativecommons.org/licenses/by-nc/4.0/), which permits any noncommercial use, distribution, and reproduction in any medium, provided you give appropriate credit to the original author(s) and the source, provide a link to the Creative Commons license, and indicate if changes were made.

\section{References}

1. Beck A, Reichert JM. Approval of the first biosimilar antibodies in Europe: a major landmark for the biopharmaceutical industry. MAbs. 2013;5(5):621-3.

2. European Medicines Agency. Guideline on similar biological medicinal products containing biotechnology-derived proteins as active substance: non-clinical and clinical issues. 2014 Dec 8. http://www.ema.europa.eu/docs/en_GB/document_library/ Scientific_guideline/2015/01/WC500180219.pdf. Accessed 21 Jun 2016.

3. WHO Expert Committee on Biological Standardization. Guidelines on evaluation of similar biotherapeutic products (SBPs). 2009 Oct 19-23. http://www.who.int/biologicals/areas/ biological_therapeutics/BIOTHERAPEUTICS_FOR_WEB_22 APRIL2010.pdf. Accessed 21 Jun 2016.

4. US Food and Drug Administration. Biosimilars: questions and answers regarding implementation of the Biologics Price Competition and Innovation Act of 2009: guidance for industry. 2015 Apr. http://www.fda.gov/downloads/drugs/guidancecom plianceregulatoryinformation/guidances/ucm444661.pdf. Accessed 28 Jul 2016.

5. Taylor L. Over 700 biosimilars now in development worldwide: report. 2014 Sep 30. http://www.pharmatimes.com/article/1409-30/over_700_biosimilars_now_in_development_worldwide_ report.aspx\#ixzz3flma1lfd. Accessed 19 Jul 2016.

6. Chastek B, White J, Van Voorhis D, Tang D, Stolshek BS. A retrospective cohort study comparing utilization and costs of biologic therapies and JAK inhibitor therapy across four common inflammatory indications in adult US managed care patients. Adv Ther. 2016;33(4):626-42.

7. Dörner T, Strand V, Cornes P, Goncalves J, Gulacsi L, Kay J, et al. The changing landscape of biosimilars in rheumatology. Ann Rheum Dis. 2016;75(6):974-82.

8. Health Canada. Summary basis of decision: Inflectra. 2015 Jun 8. http://www.hc-sc.gc.ca/dhp-mps/prodpharma/sbd-smd/drug- 
med/sbd_smd_2014_inflectra_159493-eng.php. Accessed 22 Jun 2016.

9. Pfizer Inc. Health Canada approves Inflectra ${ }^{\circledR}$ (biosimilar Infliximab) for three additional indications: Crohn's disease, fistulising Crohn's disease and ulcerative colitis. 2016 Jun 14. http://www.pfizer.ca/node/7526\#_ftnref1. Accessed 19 Jul 2016.

10. European Medicines Agency. European public assessment report: Flixabi. 2016 Apr 1. http://www.ema.europa.eu/ema/index. jsp?curl=pages/medicines/human/medicines/004020/human_med_ 001980.jsp\&mid=WC0b01ac058001d124. Accessed 19 Jul 2016.

11. Barber J. FDA advisory panel backs Amgen's proposed biosimilar of AbbVie's Humira. 2016 Jul 12. http://www. firstwordpharma.com/node/1399106\#axzz4EO2ytw2T. Accessed 19 Jul 2016.

12. Novartis. FDA accepts Sandoz regulatory submission for a proposed biosimilar etanercept. 2016. https://www.novartis.com/ news/media-releases/fda-accepts-sandoz-regulatory-submissionproposed-biosimilar-etanercept. Accessed 19 Jul 2016.

13. Pen A, Klein AV, Wang J. Health Canada's perspective on the clinical development of biosimilars and related scientific and regulatory challenges. GaBI J. 2015;4(1):36-41.

14. Jacobs I, Petersel D, Shane L, Ng C-K, Kirchhoff C, Finch G, et al. Monoclonal antibody and fusion protein biosimilars across therapeutic areas: a systematic review of published evidence. BioDrugs. doi:10.1007/s40259-016-0199-9

15. Olech E. Biosimilars: rationale and current regulatory landscape. Semin Arthritis Rheum. 2016;45(5 Suppl):S1-10.

16. Comparison of biosimilars guidelines. GaBI Journal. 2014;3(1):36-8.

17. Regulation of similar biotherapeutic products in Latin America. 2013 Aug 2. http://gabionline.net/Biosimilars/Research/ Regulation-of-similar-biotherapeutic-products-in-Latin-America. Accessed 19 Sep 2016.

18. Brennan Z. India releases new biosimilars guidance. 2016 Mar 28. http://raps.org/Regulatory-Focus/News/2016/03/28/24638/ India-Releases-New-Biosimilars-Guidance/. Accessed 19 Sep 2016.

19. Moher D, Liberati A, Tetzlaff J, Altman DG. Preferred reporting items for systematic reviews and meta-analyses: the PRISMA statement. J Clin Epidemiol. 2009;62(10):1006-12.

20. Liberati A, Altman DG, Tetzlaff J, Mulrow C, Gotzsche PC, Ioannidis JP, et al. The PRISMA statement for reporting systematic reviews and meta-analyses of studies that evaluate health care interventions: explanation and elaboration. J Clin Epidemiol. 2009;62(10):e1-34.

21. Gaffney A. After three-year delay, FDA finalizes guidance documents on biosimilarity. 2015 Apr 28.http://www.raps.org/ Regulatory-Focus/News/2015/04/28/22058/After-Three-YearDelay-FDA-Finalizes-Guidance-Documents-on-Biosimilarity/. Accessed 22 Jun 2016.

22. Jadad AR, Moore RA, Carroll D, Jenkinson C, Reynolds DJ, Gavaghan DJ, et al. Assessing the quality of reports of randomized clinical trials: is blinding necessary? Control Clin Trials. 1996;17(1):1-12.

23. Downs SH, Black N. The feasibility of creating a checklist for the assessment of the methodological quality both of randomised and non-randomised studies of health care interventions. J Epidemiol Community Health. 1998;52(6):377-84.

24. Drummond MF, Jefferson TO. Guidelines for authors and peer reviewers of economic submissions to the BMJ. The BMJ Economic Evaluation Working Party. BMJ. 1996;313(7052): 275-83.

25. Hooijmans CR, Rovers MM, de Vries RB, Leenaars M, RitskesHoitinga M, Langendam MW. SYRCLE's risk of bias tool for animal studies. BMC Med Res Methodol. 2014;14:43.
26. Kaur P, Chow V, Zhang N, Moxness M, Markus R. A randomized, single-blind, single-dose, three-arm, parallel group study in healthy subjects to demonstrate pharmacokinetic equivalence of ABP 501 and adalimumab: results of comparison with adalimumab (EU). Ann Rheum Dis. 2014;73:479.

27. Kaur PP, Chow V, Zhang N, Moxness M, Markus R. Pharmacokinetic equivalence of ABP 501 relative to adalimumab: results from a randomized, single-blind, single-dose, parallel group study in healthy subjects. Arthritis Rheum. 2014;66:S661-2.

28. Kaur P, Chow V, Zhang N, Markus R. Relationship between pharmacokinetics and anti-drug antibody status of ABP 501, a biosimilar candidate to adalimumab. Ann Rheum Dis. 2015;74:714.

29. Tatarewicz S, Miller M, Zhou L, Moxness M. Biosimilar immunogenicity assessment strategy [abstract no. M1069]. American Association of Pharmaceutical Scientists (AAPS) National Biotechnology Conference; 2-4 Nov 2014; San Diego.

30. Liu J, Hutterer K, Eris T, Abel J, Jiang Y, Li C, et al. Analytical similarity assessment of a biosimilar to adalimumab [poster no. W5350]. American Association of Pharmaceutical Scientists (AAPS) Annual Meeting; 2-4 Nov 2014; San Diego.

31. Born T, Velayudhan J, Chen Y, Thomas H, Pastula C, Maher G, et al. Demonstration of functional similarity comparing adalimumab to biosimilar candidate ABP 501 [abstract]. Arthritis Rheum. 2014;66:S661.

32. Born T, Velayudhan J, Mathur A, Chen YF. Demonstration of functional equivalence of proposed biosimilar ABP 501 to adalimumab [abstract no. P337]. 23rd European Academy of Dermatology and Venereology (EADV) Congress; 8-12 Oct 2014; Amsterdam.

33. Jani RH, Gupta RV, Bhatia G, Rathi G, Ashok Kuman P, Sharma $\mathrm{R}$, et al. A prospective, randomized, double-blind, multicentre, parallel-group, active controlled study to compare efficacy and safety of biosimilar adalimumab (Exemptia; ZRC3197) and adalimumab (Humira) in patients with rheumatoid arthritis. Int J Rheum Dis. Epub 2015 Jul 14. doi: 10.1111/1756185X.12711.

34. Shin D, Kim YH, Kim HS, Fuhr R, Körnicke T. A phase I pharmacokinetic study comparing SB5, an adalimumab biosimilar, and adalimumab reference product (Humira ${ }^{\circledR}$ ) in healthy subjects [poster no. FRI0110]. Ann Rheum Dis. 2015;74(Suppl 2):459-60.

35. Kronthaler U, Hofmann HP, Poetzl J, Demin I, Didier R, da Silva A. Characterization of efficacy and biomarker response of the proposed adalimumab biosimilar GP2017 compared to originator adalimumab in a humanized mouse model [abstract no. P338]. 23rd Congress of the European Acadamy of Dermatology and Venereology (EADV); 8-12 Oct 2014; Amsterdam.

36. Kronthaler U, da Silva A, Poetzl J, Seliskar M, Baron M. Similar preclinical pharmacokinetics of the proposed biosimilar adalimumab GP2017 and originator adalimumab upon single and multiple administrations. J Am Acad Dermatol. 2014;70:AB190.

37. Kronthaler U, Baron M, Poetzl J, Da Silva A. Preclinical PK and safety assessment of the proposed adalimumab biosimilar GP2017, compared to Humira ${ }^{\circledR}$. Ann Rheum Dis. 2014;73:943.

38. Derzi M, Ripp SL, Ng C, Shoieb AM, Finch G, Lorello LG, et al. Comparative nonclinical assessments of the potential biosimilar PF-06410293 and adalimumab [abstract no. 276]. 53rd Annual Meeting of the Society of Toxicology; 23-27 Mar 2014; Phoenix.

39. Wang X, Zhou J, Ma Q, Buckery R, Cai CHS, Chen L. Development and validation of a cell-based neutralizing antiadalimumab antibody detection methods for adalimumab 
biosimilar program [abstract no. M1023]. American Association of Pharmaceutical Scientists (AAPS) National Biotechnology Conference; 2-4 Nov 2014; San Diego.

40. Bandyopadhyay S, Mahajan M, Mehta T, Singh A, Parikh A, Gupta A, et al. Physicochemical and functional characterization of a biosimilar adalimumab ZRC-3197. Biosimilars. 2014;2015(5):1-18

41. Shin D, Kim YH, Kim YS, Fuhr R, Körnicke T. A phase I pharmacokinetic study comparing SB2, an infliximab biosimilar, and infliximab reference product $\left(\right.$ Remicade $^{\circledR}$ ) in healthy subjects [abstract]. Ann Rheum Dis. 2015;74:703.

42. Gu NY, Yi SJ, Kim T-E, Kim JW, Shin S-G, Jang I-J, et al. Comparative pharmacokinetics and tolerability of branded etanercept $(25 \mathrm{mg})$ and its biosimilar $(25 \mathrm{mg})$ : a randomized, open-label, single-dose, two-sequence, crossover study in healthy Korean male volunteers. Clin Ther. 2011;33(12):2029-37.

43. Gu NY, Kim BR, Kim BH, Kim JW, Song DH, Shin SG, et al. Comparative pharmacokinetics/tolerability of TUNEX and ENBREL in healthy Korean volunteers [poster]. Clin Pharmacol Ther. 2010;87(Suppl):S93.

44. Yi SJ, Kim SE, Park M-K, Yoon SH, Cho J-Y, Lim KS, et al. Comparative pharmacokinetics of HD203, a biosimilar of etanercept, with marketed etanercept $\left(\right.$ Enbrel $\left.^{\circledR}\right)$. BioDrugs. 2012;26(3):177-84

45. Bae S-C, Kim J, Choe J-Y, Park W, Lee SR, Ahn Y, et al. A randomized, double-blind, phase 3 equivalence trial comparing the etanercept biosimilar, HD203, with etanercept $\left(\right.$ Enbrel $\left.^{\circledR}\right)$, in combination with methotrexate (MTX) in patients with rheumatoid arthritis (RA) [abstract no. 2825]. Arthritis Rheum. 2014;66(S10):S1234.

46. Bae SC, Lee SR, Ahn Y. Quality of life assessments in Korean patients with rheumatoid arthritis (RA): an analysis from the phase III trial to evaluate equivalence of the etanercept biosimilar HD203 and Enbrel $^{\circledR}$ in combination with methotrexate (MTX) in patients with RA; the HERA study [abstract no. PMS8]. Value Health. 2014;7(17):A374.

47. Chung H, Ahn L, Choi Y, Shin S, Jang I, Yu K, et al. LBEC0101, An etanercept bisimilar, showed comparable rolerability and pharmacokinetic proles to those of etanercept in healthy male volunteers [abstract]. Clin Pharmacol Ther. 2014;95(Suppl):S39.

48. Vencovský J, Sylwestrzak A, Leszczyński P, Porawska W, Baranauskaite A, Tseluyko V, et al. A phase III randomised, double-blind clinical study comparing SB4, an etanercept biosimilar, with etanercept reference product $\left(\right.$ Enbrel $\left.^{\circledR}\right)$ in patients with moderate to severe rheumatoid arthritis despite methotrexate therapy (24-week results). Ann Rheum Dis. 2015;74:467-8

49. Emery P, Vencovský J, Sylwestrzak A, Leszczyński P, Porawska W, Baranauskaite A, et al. A phase III randomised, double-blind, parallel-group study comparing SB4 with etanercept reference product in patients with active rheumatoid arthritis despite methotrexate therapy. Ann Rheum Dis. Epub 2015 Jul 6. doi: 10.1136/annrheumdis-2015-207588.

50. Lee YJ, Shin D, Kim YH, Kang JW, Fuhr R, Gauliard A. A phase I pharmacokinetic study comparing SB4, an etanercept biosimilar, and etanercept reference product $\left(\right.$ Enbrel $\left.^{\circledR}\right)$ in healthy male subjects [poster no. SAT0176]. Ann Rheum Dis. 2015;74 Suppl 2:718.

51. da Silva A, Kronthaler U, Fritsch C, Poetzl J, Rohde A, Papandrikopoulou A, et al. Target-directed development of a proposed etanercept biosimilar, GP2015: comparability of in vitro target binding and neutralization, and in vivo efficacy and pharmacokinetics with the reference product etanercept at the pre-clinical level [abstract no. P304]. Congress of the
European Academy of Dermatology and Venereology (EADV); 2-6 Oct 2013; Istanbul.

52. Maity S, Ullanat R, Lahiri S, Shekar S, Sodhan G, Vyas A, et al. A non-innovator version of etanercept for treatment of arthritis. Biologicals. 2011;39(6):384-95.

53. da Silva A, Kronthaler U, Fritsch C, Poetzl J, Rohde A, Papandrikopoulou A, et al. Target-directed development of a proposed biosimilar etanercept, GP2015: Comparability of in vitro target binding and pre-clinical efficacy and pharmacokinetics [abstract]. Arthritis Rheum. 2013;65:S794.

54. Kay J, Lassen C, Trokan L, Wyand M. Safety profile of BOW015, a biosimilar infliximab, in healthy subjects and patients with active rheumatoid arthritis [poster]. Ann Rheum Dis. 2015;74(Suppl 2):706.

55. Lambert J, Wyand M, Lassen C, Shneyer L, Thomson E, Kay J. Pharmacokinetic results from a phase 1 , single-centre, doubleblind, randomised, single-dose, parallel group study comparing $5 \mathrm{MG} / \mathrm{KG}$ IV infusion of BOW015 and reference infliximab in healthy male volunteers [poster]. Ann Rheum Dis. 2015;74(Suppl 2):462.

56. Kay J, Chopra A, Lassen C, Shneyer L, Wyand M. BOW015, a biosimilar infliximab: disease activity and disability outcomes from a phase 3 active comparator study in ptients with active rheumatoid arthritis on stable methotrexate doses [poster no. FRI0117]. Ann Rheum Dis. 2015;74(Suppl 2):462-3.

57. Kay J, Chopra A, Chandrashekara S, Olakkengil D, Bhojani K, Bhatia G, et al. Secondary efficacy outcomes from a phase 3 study support clinical equivalence between BOW015 and infliximab in patients with active rheumatoid arthritis on stable methotrexate doses [abstract no. AB0420]. Ann Rheum Dis. 2015;74(Suppl 2):1034.

58. Park W, Hrycaj P, Kovalenko V, Miranda P, Gutierrez-Ureña S, Lee $\mathrm{Y}$, et al. A randomized, double-blind, phase 1 study demonstrates equivalence in pharmacokinetics, safety, and efficacy of CT-P13 and infliximab in patients with ankylosing spondylitis [abstract no. OP0167]. Ann Rheum Dis. 2012;71(Suppl 3):111.

59. Park W, Hrycaj P, Jeka S, Kovalenko V, Lysenko G, Miranda P, et al. A randomised, double-blind, multicentre, parallel-group, prospective study comparing the pharmacokinetics, safety, and efficacy of CT-P13 and innovator infliximab in patients with ankylosing spondylitis: the PLANETAS study. Ann Rheum Dis. 2013;72(10):1605-12.

60. Park W, Jaworski J, Brzezicki J, Gnylorybov A, Kadinov V, Goecke Sariego I, et al. A randomised, double-blind, parallelgroup, phase 1 study comparing the pharmacokinetics, safety and efficacy of CT-P13 and infliximab in patients with active ankylosing spondylitis: 54 week results from the PLANETAS study [poster no. FRI0421]. Ann Rheum Dis. 2013;72(Suppl 3):A516-7.

61. Park W, Miranda P, Brzosko M, Wiland P, Gutierrez-Urena S, Mikazane H, et al. Efficacy and safety of CT-P13 (infliximab biosimilar) over two years in patients with ankylosing spondylitis: comparison between continuing with CT-P13 and switching from infliximab to CT-P13 [abstract no. L15]. Arthritis Rheum. 2013;65:3326.

62. Park W, Yoo DH, Szántó S, Berghea F, Brzosko M, Wiland P, et al. Clinical response of disease activity, disability and mobility indices in relation to anti-drug antibody in the PLANETAS [abstract no. OP0157]. Ann Rheum Dis. 2014;73(Suppl 2):121.

63. Park W, Yoo D, Hrycaj P, Prodanovic N, Miranda P, Ramiterre $\mathrm{E}$, et al. The rate of positive conversion in the quantiferon-TB gold test over 2 years among patients treated with CT-P13 or innovator infliximab in the extension studies of PLANETAS and PLANETRA [abstract]. Ann Rheum Dis. 2014;73(Suppl 2):485. 
64. Braun J, Baraliakos X, Kudrin A, Kim H, Lee SJ. Striking discrepancy in the development of anti-drug antibodies (ADA) in patients with rheumatoid arthritis (RA) and ankylosing spondylitis (AS) in response to infliximab (INF) and its biosimilar CT-P13 [abstract]. Arthritis Rheum. 2014;66:3538-9.

65. Braun J, Park W, Yoo DH, Suh CH, Shim SC, Lee SJ, et al. What intrinsic and extrinsic factors affect the developement of anti-drug antibody to innovator infliximab and its biosimilar CTP13 in rheumatoid arthritis and ankylosing spondylitis [poster no. FRI0119]. Ann Rheum Dis. 2015;74(Suppl 2):463-4.

66. Lee SJ, Yoo DH, Park W, Müller-Ladner U, Pyo TN. Statistical evaluation of joint damage progression in patients with rheumatoid arthritis treated with infliximab or biosimilar infliximab (CT-P13) anti-TNF therapy: a role of sensitivity analysis for missing data evaluating similarity [abstract no. SAT0214]. Ann Rheum Dis. 2014;73(Suppl 2):667.

67. Yoo D, Miranda P, Piotrowski M, Ramiterre E, Kovalenko V, Prodanovic N, et al. A randomized, double-blind, phase 3 study demonstrates clinical equivalence of CT-P13 to infliximab when co-administered with methotrexate in patients with active rheumatoid arthritis [abstract no. FRI0143]. Ann Rheum Dis. 2012;71(Suppl 3):359.

68. Yoo DH, Shevchuk S, Ramiterre E, Kovalenko V, Tee M, Tobias Arteaga E, et al. Local tuberculosis incidence affects the rate of positive conversion in the quantiferon ${ }^{\circledR}$-tb gold test among patients receiving infliximab or CT-P13 therapy [poster no. FRI0164]. Ann Rheum Dis. 2013;72(Suppl 3):426-7.

69. Yoo DH, Hrycaj P, Miranda P, Ramiterre E, Piotrowski M, Shevchuk S, et al. A randomised, double-blind, parallel-group study to demonstrate equivalence in efficacy and safety of CTP13 compared with innovator infliximab when coadministered with methotrexate in patients with active rheumatoid arthritis: the PLANETRA study. Ann Rheum Dis. 2013;72(10):1613-20.

70. Yoo DH, Racewicz A, Brzezicki J, Yatsyshyn R, Arteaga ET, Baranauskaite A, et al. A phase 3 randomised controlled trial to compare CT-P13 with infliximab in patients with active rheumatoid arthritis: 54 week results from the PLANETRA study [oral presentation no. OP0068]]. Ann Rheum Dis. 2013;72(Suppl 3):A73.

71. Yoo DH, Yagensky A, Toncheva A, Ruiz Santacruz O, Cons Molina F, Bae Y, et al. Impact of CT-P13 and originator infliximab treatment on quality of life derived from the health assessment questionnaire (HAQ) and short-form 36 (SF-36) from a randomized, double-blind trial in patients with active RA [abstract]. Arthritis Rheum. 2013;65(suppl 10):2392.

72. Yoo DH, Prodanovic N, Jaworski J, Miranda P, Ramiterre EB, Lanzon A. Efficacy and safety of CT-P13 (infliximab biosimilar) over two years in patients with rheumatoid arthritis: comparison between continued CT-P13 and switching from infliximab to CT-P13 [abstract no. L1]. American College of Rheumatology/ Association of Rheumatology Health Professionals (ACR/ ARHP) Annual Meeting; 25-30 Oct 2013; San Diego.

73. Yoo D, Park W, Miranda P, Piotrowski M, Ramiterre E, Shevchuk S, et al. Inhibition of radiographic progression and its association with clinical parameters in RA patients treated with CT-P13 and innovator infliximab in PLANETRA study [abstract no. THU0158]. Ann Rheum Dis. 2014;73(Suppl 2):234-5.

74. Molnar T, Farkas K, Rutka M, Balint A, Nagy F, Bor R, et al. Efficacy of the new infliximab biomarker CT-P13 induction therapy on mucosal healing in ulcerative colitis patients [poster no. P603]. J Crohns Colitis. 2015;9(Suppl 1):S382.

75. Yoon Suk J, Park DI, Kim YH, Seo PJ, Kim JW, et al. Efficacy and safety of infliximab's biosimilar $\left(\right.$ Remsima $\left.^{\circledR}\right)$ for IBD. J Crohns Colitis. 2015;9(Suppl 1):S340-50.

76. Jung YS, Park DI, Kim YH, Lee JH, Seo PJ, Cheon JH, et al. Efficacy and safety of CT-P13, a biosimilar of infliximab, in patients with inflammatory bowel disease: a retrospective multicenter study. J Gastroenterol Hepatol. 2015;30(12):1705-12.

77. Kang HW, Lim YJ, Kim JH, Kang Y-S. An experience of antiTNF biosimilar, CT-P13 use: clinical efficacy, safety and interchangeability in inflammatory bowel disease. A pilot study [abstract no. P565]. J Crohns Colitis. 2014;8(Suppl):S303.

78. Kang Y-S, Moon HH, Lee SE, Lim YJ, Kang HW. Clinical experience of the use of CT-P13, a biosimilar to infliximab in patients with inflammatory bowel disease: a case series. Dig Dis Sci. 2015;60(4):951-6.

79. Murphy C, Sugrue K, Mohamad G, McCarthy J, Buckley M. Biosimilar but not the same [abstract no. P505]. J Crohns Colitis. 2015;9(Suppl 1):S331-2.

80. Farkas K, Rutka M, Bálint A, Nagy F, Bor R, Milassin Á, et al. Efficacy of the new infliximab biosimilar CT-P13 induction therapy in Crohn's disease and ulcerative colitis-experiences from a single center. Expert Opin Biol Ther. 2015;15(9):1257-62.

81. Gecse K, Farkas K, Lovász B, Banai J, Bene L, Gasztonyi B, et al. Biosimilar Infliximab in inflammatory bowel diseases: first interim results from a prospective nationwide observational cohort [abstract]. Z Gastroenterol. 2015;53(5):A11.

82. Kierkus J. Preliminary assessment of efficacy and safety of switching between originator and biosimilar infliximab in paediatric Crohn disease patients. Gastroenterology. 2015;148(4 Suppl 1):S782-3.

83. Kim J, An Hong J, Kudrin A. 5 year budget impact analysis of CT-P13 (infliximab) for the treatment of Crohn's Disease in UK, Italy and France [poster no. P137]. J Crohns Colitis. 2015;9(Suppl 1):S144-5.

84. Brodszky V, Baji P, Balogh O, Péntek M. Budget impact analysis of biosimilar infliximab (CT-P13) for the treatment of rheumatoid arthritis in six Central and Eastern European countries. Eur J Health Econ. 2014;15(1):65-71.

85. Brodszky V, Gulacsi L, Balogh O, Baji P, Rencz F, Péntek M. Budget impact analysis of biosimilar infliximab for the treatment of Crohn's disease in six Central Eastern European countries [abstract]. Value Health. 2014;17(7):A364.

86. Jha A, Upton A, Dunlop WCN. Budget impact analysis of introducing biosimilar infliximab for the treatment of auto immune disorders in five European countries [abstract]. Value Health. 2014;17(7):A525.

87. Jha A, Upton A, Dunlop WCN, Akehurst R. The budget impact of biosimilar infliximab (Remsima ${ }^{\circledR}$ ) for the treatment of autoimmune diseases in five European countries. Adv Ther. 2015;32(8):742-56.

88. Kim J, Hong J, Kudrin A. 5 Year budget impact analysis of biosimilar infliximab for the treatment of rheumatoid arthritis in UK, Italy, France and Germany [abstract]. Arthritis Rheum. 2014;66:S512.

89. McCarthy G, Bitoun CE, Guy H. Introduction of an infliximab biosimilar (CT-P13): a five-year budget impact analysis for the treatment of rheumatoid arthritis in Ireland [abstract no. PM522]. Value Health. 2013;7(16):A558.

90. Jung SK, Lee KH, Jeon JW, Lee JW, Kwon BO, Kim YJ, et al. Physicochemical characterization of Remsima ${ }^{\circledR}$. MAbs. 2014;6:1163-77.

91. Andrick B, Stroyne K, Kamal K, Meng W. Predicting immunogenicity of infliximab: pharmacoeconomic implications for biosimilars [poster no. e185]. American Pharmacists Association (APhA) 2014 Annual Meeting and Exposition; 28-31 Mar 2014; Orlando.

92. Pierri CL, Cetrone M, Punzi G, Tricarico D. Therapeutic antiTNF alpha antibodies: structure-activity investigations [abstract]. FASEB J. 2015;29(1 Suppl):941.9. 
93. Sharpe P, Saati A, Koob S, Gu L, Aggarwal P, Porter T, et al. Characterization and similarity assessment of infliximab and PF06438179: a proposed biosimilar [abstract no. T2022]. American Association of Pharmaceutical Scientists (AAPS) National Biotechnology Conference; 2-4 Nov 2014; San Diego.

94. Johnson T, Derzi M, Bolt M, Conlon H, Kirchhoff C, Lorello L, et al. Comparative nonclinical assessments of marketed infliximab presentations (Remicade) and the potential biosimilar PF06438179 [poster no. W3065]. American Association of Pharmaceutical Scientists (AAPS) National Biotechnology Conference; 2-4 Nov 2014; San Diego.

95. McClellan JE, Udata C, Yin D, Salts S, Johnson TR, Derzi M, et al. Comparative structural, functional, nonclinical, and phase 1 similarity assessments of PF-06438179, a potential biosimilar to infliximab, and marketed reference products. J Crohns Colitis. 2015;9(Suppl 1):S94-5.

96. Yin D, Udata C, Hua S, Salts S, Meng X, Johnson TR, et al. Comparative assessments of PF-06438179, a potential biosimilar, and infliximab in a phase 1 pharmacokinetic study [abstract]. Gastroenterology. 2015;148(4 Suppl 1):S-642.

97. Udata C, Yin D, Cai C-H, Hua SY, Salts S, Rehman MI, AlSabbagh A, McClellan J, Meng X. Immunogenicity assessment of PF-06438179, a potential biosimilar to infliximab, in healthy volunteers [poster]. Ann Rheum Dis. 2015;74(Suppl 2):702.

98. Udata C, Hua SY, Yin D, Salts S, Meng X, Rehman MI. A phase I pharmacokinetics trial comparing PF-06438179 (a potential biosimilar) and infliximab in healthy volunteers (REFLEXTIONS B537-01) [abstract]. Ann Rheum Dis. 2014;73(Suppl 2):494.

99. Udata C, Yin D, Cai C-H, Salts S, Hua SY, Rehman MI, et al. Immunogenicity assessment of PF-06438179, a potential biosimilar to infliximab, in healthy volunteers [poster]. Arthritis Rheum. 2014;66(Suppl 2):S660.

100. Choe JY, Prodanovic N, Niebrzydowski J, Staykov I, Dokoupliova E, Baranauskaite A, et al. A randomised, double-blind, phase III study comparing SB2, an infliximab biosimilar, to the infliximab reference product (Remicade $\AA$ ) in patients with moderate to severe rheumatoid arthritis despite methotrexate therapy. Ann Rheum Dis. 2015;74:706-7.

101. Choe J-Y, Prodanovic N, Niebrzydowski J, Staykov I, Dokoupilova E, Baranauskaite A, et al. A randomised, double-blind, phase III study comparing SB2, an infliximab biosimilar, to the infliximab reference product Remicade in patients with moderate to severe rheumatoid arthritis despite methotrexate therapy. Ann Rheum Dis. Epub 2015 Aug 28. doi: 10.1136/annrheumdis2015-207764.

102. Yoo DH, Park W, Jeka S, Molina FC, Hrycaj P, Wiland P, et al. A randomized, controlled, multicenter, 2-arm, parallel-group, double-blind study to demonstrate the equivalence of CT-P10 to innovator rituximab with respect to pharmacokinetic profile in patients with rheumatoid arthritis [abstract]. Arthritis Rheum. 2013;65(suppl 10): 1736 .

103. Yoo DH, Park W, Jeka S, Cons Molina F, Hrycaj P, Wiland P, et al. Impact of anti-drug antibody on efficacy and safety over week 24 in both CT-P10 and innovator rituximab treatment groups [abstract]. Arthritis Rheum. 2014;65(suppl 10):S736.

104. Becker J-CP, Yin D, Melia LA, Li R, Gumbiner B, Thomas D, et al. A phase I trial comparing PF-05280586 (a potential biosimilar) and rituximab in subjects with active rheumatoid arthritis [abstract]. Arthritis Rheum. 2014;66:S660-1.

105. Yin D, Becker J-C, Melia L, Li R, Gumbiner B, Thomas D, et al. A phase I pharmacokinetics trial comparing PF-05280586 (a potential biosimilar) and rituximab in subjects with active rheumatoid arthritis with active disease in TNF failures (REFLECTIONS B328-01) [abstract no. FRI0309]. Ann Rheum Dis. 2014;73(Suppl 2):497.
106. Jacobs I, Yin D, Melia LA, Gumbiner B, Suster M, Thomas D, et al. A phase I trial comparing PF-05280586 (a potential biosimilar) and rituximab in patients with active rheumatoid arthritis [poster no. SAT0190]. Ann Rheum Dis. 2015;74(Suppl 2): 724 .

107. Williams J, Hutmacher MH, Zierhut M, Becker J, Gumbiner B, Spencer-Green G, et al. Assessment of clinical response in patients with rheumatic arthritis (RA) between PF-05280586, a proposed biosimilar to rituximab and two rituximab products [abstract]. Clin Pharmacol Ther. 2015;97:S96.

108. Ryan AM, Sokolowski SA, Ng C-K, Shirai N, Collinge M, Shen AC, et al. Comparative nonclinical assessments of the proposed biosimilar PF-05280586 and rituximab (MabThera ${ }^{\circledR}$ ). Toxicol Pathol. 2014;42(7):1069-81.

109. Hurst SI, Ryan AM, Ng C-K, Ploch SA, Finch GL, Leach MW. Nonclinical assessments demonstrating the similarity of the proposed biosimilar PF-05280586 and rituximab [abstract]. Ann Rheum Dis. 2013;72:A198.

110. Hurst S, Ryan AM, Ng CK, Thompson M, Plock S, Finch GL, et al. Comparative nonclinical assessments of rituximab-EU (MabThera ${ }^{\mathrm{B}}$ ) and the potential biosimilar PF-05280586 [abstract no. M1125]. American Association of Pharmaceutical Scientists (AAPS) National Biotechnology Conference; 20-23 May 2013; San Diego.

111. Karnik S, Thompson M, De Gruttola H, Ferrari S, Gu L, Aggarwal P, et al. Characterization and comparison of PF05280586-a proposed rituximab biosimilar to the licensed product [poster no. M1038]. American Association of Pharmaceutical Scientists (AAPS) National Biotechnology Conference; 20-23 May 2013; San Diego.

112. Thomas D, Becker J-CP, Ng C-K. Comparison of proposed biosimilar PF-05280586 with rituximab: nonclinical and phase I clinical assessments [abstract]. Arthritis Rheum. 2013;65:S1014.

113. Ryan AM, Sokolowski S, Collinge M, Shen A, Arrington J, Cummings $\mathrm{T}$, et al. Comparative pharmacodynamic effects of rituximab-EU (MabThera ${ }^{\circledR}$ ) and rituximab-Pfizer in cynomolgus monkeys [poster no. 1800]. 52nd Annual Meeting and ToxExpo of the Society of Toxicology (SOT); 10-14 Mar 2013; San Antonio.

114. Hurst S, Ryan A, Ng C, Thompson M, McNally J, Ploch S, et al. Comparative nonclinical assessments of trastuzumab-US and trastuzumab-EU (Herceptin ${ }^{\circledR}$ ) and the potential biosimilar PF05280014 [poster no. M1125]. American Association of Pharmaceutical Scientists National Biotechnology Conference; 20-23 May 2013; San Diego.

115. da Silva A, Kronthaler U, Koppenburg V, Fink M, Meyer I, Papandrikopoulou A, et al. Target-directed development and preclinical characterization of the proposed biosimilar rituximab GP2013. Leuk Lymphoma. 2014;55(7):1609-17.

116. da Silva A, Kronthaler U, Meyer I, Fritsch C, Schneiderer T, Stangler $\mathrm{T}$, et al. Physicochemical, functional, and pharmacologic comparability between the proposed biosimilar rituximab GP2013 and originator rituximab as the foundation for biosimilarity [abstract]. J Clin Oncol. 2013;31(Suppl): Abstr 3075

117. Visser JM, Feuerstein I, Stangler T, Schmiederer T, Fritsch C, Schiestl M. Physicochemical and functional comparability between the proposed biosimilar rituximab GP2013 and originator rituximab. BioDrugs. 2013;27(5):495-507.

118. Seigelchifer M, Corley E, Fresnillo G, Pesce A, Bes C, Elise M, et al. Development of RTXM83 (a potential rituximab biosimilar): in vitro and in vivo comparability with MabThera [abstract e14020]. J Clin Oncol. 2014;32(Suppl):e14020.

119. Kay J, Chopra A, Chandrashekara S, Olakkengil DJ, Bhojani KS, Bhatia G, et al. A phase 3, randomized, double-blind, active comparator study of the efficacy and safety of BOW015, a biosimilar infliximab, in patients with active rheumatoid arthritis 
on stable methotrexate doses [abstract no. OP0012]. Ann Rheum Dis. 2014;73(Suppl 2):64.

120. Kay J, Wyand M, Chandrashekara S, Olakkengil DJ, Bhojani K, Bhatia G, et al. BOW015, a biosimilar infliximab, in patients with active rheumatoid arthritis on stable methotrexate doses: 54-week results of a randomized, double-blind, active comparator study [abstract no. FRI0117]. Arthritis Rheum. 2014;66(Suppl 2):3538.

121. Yoo DH. Disease activity assessment using the DAS28, CDAI and SDAI and effect of anti-drug antibody on clinical response in a randomised, double-blind, comparative trial of CT-P13 and the innovator infliximab: PLANETRA study [abstract no. THU0159]. Ann Rheum Dis. 2014;72(Suppl 2):235.

122. Codreanu C, Mogosan C, Enache L, Stanciu D, Ionescu R, Opris D, et al. Romanian Registry of Rheumatic Diseases: efficacy and safety of biologic therapy in rheumatoid arthritis [poster no. FRI0107]. Ann Rheum Dis. 2015;74(Suppl 2):458.

123. Park W, Yoo DH, Suh CH, Shim SC, Lee SJ, Lee SY, et al. Meta-analysis of the safety data between infliximab biosimilar (CT-P13) and innovator infliximab in rheumatoid arthritis and ankylosing spondylitis [abstract no. AB0433]. Ann Rheum Dis. 2015;74(Suppl 2):1039.

124. Moctezuma JF, Martinez A, Enkerlin H, Garcia C, Chavez B, Salazar-Teran N, et al. Comparative, randomized, simple blind to evaluate efficacy and safety of Infinitam $^{\circledR}$ (etanercept), associated with methotrexate compared with Enbrel $^{\circledR}$ (etanercept) associated with methotrexate in patients with moderate and severe rheumatoid arthritis [poster no. THU0208]. Ann Rheum Dis. 2013;72:A234

125. Barile-Fabris LA, Irazoque-Palazuelos F, Hernandez Vasquez R, Carrillo Vazquez S, Guzman R. Incidence of adverse events in patients treated with intended copies of biologic therapeutic agents in Colombia and Mexico [abstract]. Arthritis Rheum. 2014;66:S662.

126. Santos-Moreno P, Saavedra-Martinez G, Villarreal L, Gomez D, Bello-Gualtero J, Giraldo V, et al. Etanar-an etanercept biosimilar is as effective as adalimumab and infliximab in a cohort of real-life of patients with rheumatoid arthritis. Ann Rheum Dis. 2015;74:789-90.

127. Wu B, Song Y, Leng L, Bucala R, Lu LJ. Treatment of moderate rheumatoid arthritis with different strategies in a health resource-limited setting: a cost-effectiveness analysis in the era of biosimilars. Clin Exp Rheumatol. 2015;33(1):20-6.

128. Bandyopadhyay S. Safety and efficacy of rituximab-biosimilar for the treatment of moderate to severe rheumatoid arthritis patients following the failure of disease-modifying drugs: a case series from Apollo Gleneagles Hospital, Kolkata. Indian J Rheumatol. 2013;8(5 Suppl):S4-S5.

129. Bandyopadhyay S. Efficacy and safety of rituximab-biosimilar in rheumatoid arthritis. Int J Rheumatic Diseas. 2014;17(Suppl S1):57.

130. Aliaga L, Fernandez I, Sanchez M, Saavedra H, Espinoza C. Pharmacovigilance of anti CD 20 monoclonal antibody biosimilar at the Edgardo Rebagliati Martins Hospital-Peru [abstract]. Drug Saf. 2013;36:925.

131. Roshique KK, Ravindran V. Efficacy and safety of a biosimilar rituximab in biologic naive patients with active rheumatoid arthritis. Clin Rheumatol. 2015;34(7):1289-92.

132. Flores-Ortiz LF, Campos-García VR, Perdomo-Abúndez FC, Pérez NO, Medina-Rivero E. Physicochemical properties of rituximab. J Liq Chromatogr Relat Technol. 2014;37(10):1438-52.

133. Kumar V, Vemuri S, Lella RK, Babu MN, Atluri A, Gowravaram M, et al. Suitability of 'one assay' for immunogenicity assessment of both proposed biosimilar and innovator rituximab [poster no.M1042]. American Association of Pharmaceutical
Scientists National Biotechnology Conference; 2-4 Nov 2014; San Diego.

134. Mekhssian K, Mess JN, Montalibet J, Garofolo F. Application of complementary HRMS methodologies for a thorough biosimilar comparability assessment [poster no. T2055]. American Association of Pharmaceutical Scientists (AAPS) National Biotechnology Conference; 8-10 Jun 2015; San Francisco.

135. Lin S, Ramatlapeng D, Hanning J, Bakewell W. Comparability study of monoclonal biosimilar drug products using various analytical methods [abstract]. 18th Symposium on the Interface of Regulatory and Analytical Sciences for Biotechnology Health Products (CASSS WCBP); 28-30 Jan 2014; Washington, DC.

136. US Food and Drug Administration. Fact sheet: issuance of draft guidances on biosimilar product development. 2012 Sep. http:// www.fda.gov/Drugs/DevelopmentApprovalProcess/HowDrugsare DevelopedandApproved/ApprovalApplications/TherapeuticBio logicApplications/Biosimilars/ucm291197.htm. Accessed 28 Jul 2016.

137. European Medicines Agency. Guideline on similar biological medicinal products containing biotechnology-derived proteins as active substance: non-clinical and clinical issues. 2014 Dec 18. http://www.ema.europa.eu/docs/en_GB/document_library/ Scientific_guideline/2015/01/WC500180219.pdf. Accessed 19 Sep 2016.

138. US Food and Drug Administration. Scientific considerations in demonstrating biosimilarity to a reference product. Guidance for industry. 2015 Apr. http://www.fda.gov/downloads/drugs/ guidancecomplianceregulatoryinformation/guidances/ucm291128. pdf. Accessed 19 Sep 2016.

139. Braun J, Kudrin A. Progress in biosimilar monoclonal antibody development: the infliximab biosimilar CT-P13 in the treatment of rheumatic diseases. Immunotherapy. 2015;7(2):73-87.

140. Miedany YE, Gaafary ME, Ahmed I, Youssef S, Nasr A. US guided treat-to-target approach in early RA: implications for uncoupling of disease activity and structural joint damage. Curr Rheumatol Rev. Epub 2015 May 21.

141. Breedveld F. The value of early intervention in RA-a window of opportunity. Clin Rheumatol. 2011;30(Suppl 1):S33-9.

142. Herrera-deGuise C, Casellas F, Robles V, Navarro E, Borruel N. Predictive value of early restoration of quality of life in Crohn's disease patients receiving antitumor necrosis factor agents. J Gastroenterol Hepatol. 2015;30(2):286-91.

143. Jahnsen J. Clinical experience with infliximab biosimilar Remsima (CT-P13) in inflammatory bowel disease patients. Therap Adv Gastroenterol. 2016;9(3):322-9.

144. Dörner T, Kay J. Biosimilars in rheumatology: current perspectives and lessons learnt. Nat Rev Rheumatol. 2015;11(12):713-24.

145. Castañeda-Hernández G, González-Ramírez R, Kay J, Scheinberg MA. Biosimilars in rheumatology: what the clinician should know. RMD Open. 2015;1(1):e00010.

146. Declerck PJ. Biologicals and biosimilars: is similar the same? J Crohns Colitis. 2014;8(Suppl 1):S427.

147. Annese V, Vecchi M. Use of biosimilars in inflammatory bowel disease: statements of the Italian Group for Inflammatory Bowel Disease. Dig Liver Dis. 2014;46(11):963-8.

148. Goel N, Chance K. The biosimilar landscape: a systematic review of its current status [abstract]. Arthritis Rheum. 2014;74:S662.

149. Scott BJ, Klein AV, Wang J. Biosimilar monoclonal antibodies: a Canadian regulatory perspective on the assessment of clinically relevant differences and indication extrapolation. J Clin Pharmacol. 2015;55(Suppl 3):S123-32.

150. Hlavaty T, Letkovsky J. Biosimilars in the therapy of inflammatory bowel diseases. Eur J Gastroenterol Hepatol. 2014;26(6):581-7. 
151. Ainsworth M. Is extrapolation of safety and efficacy data possible? Karger. 2015;34:107-12.

152. Gomollon F. Biosimilars: are they bioequivalent? Dig Dis. 2014;32(Suppl 1):82-7.

153. Gomollon F. Biosimilars in inflammatory bowel disease: ready for prime time? Curr Opin Gastroenterol. 2015;31(4):290-5.

154. Lee H. Is extrapolation of the safety and efficacy data in one indication to another appropriate for biosimilars? AAPS J. 2014;16(1):22-6.

155. Puig L. Biosimilars in dermatology: starting with infliximab. Actas Dermosifiliogr. 2013;104(3):175-80.

156. Puig L, Carretero G, Dauden E, Ferrandiz C, Marron SE, Martorell A, et al. Biosimilars in dermatology: current situation (Part I). Actas Dermosifiliogr. 2015;106(7):545-9.

157. Puig L, Carretero G, Dauden E, Ferrandiz C, Marron SE, Martorell A, et al. Biosimilars in dermatology: current situation (Part II). Actas Dermosifiliogr. 2015;106(7):550-4.

158. Teixeira FV, Kotze PG, Damiao AO, Miszputen SJ. Biosimilars in inflammatory bowel diseases: an important moment for
Brazilian gastroenterologists. Arq Gastroenterol. 2015;52(1): 76-80.

159. Schellekens H, Lietzan E, Faccin F, Venema J. Biosimilar monoclonal antibodies: the scientific basis for extrapolation. Expert Opin Biol Ther. 2015;15(11):1633-46.

160. Feagan BG, Choquette D, Ghosh S, Gladman DD, Ho V, Meibohm B, et al. The challenge of indication extrapolation for infliximab biosimilars. Biologicals. 2014;42(4):177-83.

161. Epirus Biopharmaceuticals. EPIRUS announces reprioritization of pipeline to solely focus on biosimilars to treat rare diseases and key leadership changes. 2016 May 9. https://www.sec.gov/ Archives/edgar/data/1135906/000110465916119230/a16-10868_ 1ex99d1.htm. Accessed 19 Jul 2016.

162. Hanwha to transfer biosimilar etanercept technology to Merck. GaBi Online. 2015 Feb 6. http://www.gabionline.net/Biosimilars/ News/Hanwha-to-transfer-biosimilar-etanercept-technology-toMerck. Accessed 19 Sep 2016. 